\title{
Molecular Microwave Spectra Tables ${ }^{1}$
}

\author{
By Paul Kisliuk ${ }^{2}$ and Charles H. Townes ${ }^{2}$
}

\begin{abstract}
This paper presents a group of tables that give the frequencies, assignment of quantum numbers, and intensities of over 700 microwave absorption lines. The best available values of other pertinent molecular data, such as moments of inertia, dipole moments, quadrupole coupling constants, and rotation-vibration constants are also included. The frequencies are listed once for each molecule and again in consecutive ascending order of frequency. References are given for all data included. Frequencies listed to the nearest megacycle were generally measured with a cavity wave meter and may be in error by as much as 10 megacycles, whereas those given to a fraction of a megacycle are generally known to an accuracy of about 0.1 megacycle.

A short discussion of microwave spectra and important formulas is given. For easy calculation of hyperfine structure Casimir's function is tabulated up to $J=10$ and $I=9 / 2$.
\end{abstract}

\section{Introduction}

These tables were undertaken because of the interest and encouragement of a number of workers in microwave spectroscopy. Beside encouragement, our colleagues have provided a considerable amount of unpublished information so that the tables could be as complete and up-todate as possible. However, the considerable activity in microwave spectroscopy makes obsolescence of the present tables inevitable, and it is expected that they will be revised and republished from time to time. Suggestions or additions for future editions of these tables will be gladly received, either by the authors or the Microwave Standards Section, National Bureau of Standards, Washington, D. C. A supplement to bring these tables up to date will soon be issued. It is especially hoped that information obtained on microwave spectral lines that is not otherwise published will be received so that it may be included in the tables and thus made available.

Only molecular lines of frequency greater than 1,000 megacycles have been listed. This excludes nuclear resonances found by molecular beam techniques rather than the usual microwave absorption measurements, as well as the lines of atomic hydrogen and cesium that fall in the microwave region. All lines reported in the

\footnotetext{
1 Work supported jointly by the Signal Corps, the Office of Naval Re-
search, and the National Bureau search, and the National Bureau of Standards.

2 Physics Department, Columbia University.
}

literature or otherwise available prior to February 1949 have been listed. In addition to those actually observed and listed, a large number of other lines may readily be predicted from the information provided.

The primary information is presented in an alphabetic listing of the molecules by isotopic species, which is subdivided according to the transition, except for the hyperfine components, which are presented in order of frequency. With the listing of each molecule, all available molecular constants are given that are needed in interpreting the spectra. These include moments of inertia, dipole moments, quadrupole coupling constants, and rotation-vibration constants. In addition to the observed frequency and the transition assigned to each line, we have included a calculated value for the intensity and references to the latest sources of data from each laboratory that has measured the line. The most precise reported values of the frequencies were used, or the average if there seemed no reason for preference. Frequencies listed to the nearest megacycle were generally measured with a cavity wavemeter and are subject to an error of 10 or more megacycles, whereas those given to a fraction of a megacycle are generally known to an accuracy of about 0.1 megacycle.

Another listing of all the lines in order of frequency is included, with sufficient information to locate the line in the first table and a classification 
of the line as strong, medium, or weak. We have arbitrarily taken lines of maximum absorption coefficient less than $5 \times 10^{-7} \mathrm{~cm}^{-1}$ as weak, those from $5 \times 10^{-7}$ to $10^{-5} \mathrm{~cm}^{-1}$ as medium, and those greater than $10^{-5} \mathrm{~cm}^{-1}$ as strong.

The general characteristics of the microwave spectra of most molecules may be explained on the basis of a rigid rotator model. Molecules are conveniently divided into three classes; linear molecules, symmetric tops, and asymmetric tops [26]. ${ }^{3} \quad$ For linear molecules the frequency of pure rotational lines is given to a good approximation by: [26]

$$
\begin{gathered}
\nu_{J \rightarrow J+1}=2(J+1) B_{v}-4 D_{v}\left\{(J+1)^{3}-(J+1) l^{2}\right\} \sec ^{-1} \\
\text { and } B_{v}=\frac{h}{8 \pi^{2}}\left\langle\frac{1}{I}\right\rangle_{A v .}=B_{e}-\sum_{i} \alpha_{i}\left(v_{i}+\frac{d_{i}}{2}\right)
\end{gathered}
$$

where $J$ is the total angular momentum quantum number,

$l$ is the angular momentum quantum number along the figure axis (zero for the ground vibrational state),

$D_{v}$ is the centrifugal distortion coefficient in $\sec ^{-1}$,

$B_{v}$ is the average reciprocal moment of the molecule in $\sec ^{-1}$,

$B_{e}$ is the reciprocal moment in $\sec ^{-1}$ if the nuclei were in the equilibrium position,

$\alpha_{i}$ is a coefficient of the change in reciprocal moment per quantum of excitation of the $i^{\text {th }}$ vibrational state, and $v_{i}$ and $d_{i}$ are the corresponding quantum number and degree of degeneracy.

In addition there is a splitting term for the degenerate vibrational states [53]. To a somewhat rougher approximation the frequencies for both linear molecules and symmetric tops is given by $\nu=2 B(J+1)$, where $B$ is a slowly varying function of the quantum numbers. The case of the asymmetric top is complex, and is discussed in reference $[9,29]$ and earlier papers quoted therein.

In the event that one or more of the nuclei has a quadrupole moment different from zero, the resulting coupling to the molecular electric field splits the energy levels into a hyperfine structure which depends on the various possible orientations

3 Figures in brackets indicate the literature references at the end of this
paper.

${ }^{3}$ Figures in brackets indicate the literature references at the end of this
paper. of the nuclear spin. The interaction between nuclear magnetic moments and molecular magnetic fields is usually much smaller, and has thus far been observed only as a small correction in the ammonia spectrum. The quadrupole interaction for the case of a single nucleus in a linear or symmetric top molecule is given by references $[5,7 \mathrm{a}$, and 58a]. The energies are given by

$$
\begin{aligned}
& E_{Q}=(e Q q) \\
& \left\{\frac{3 K^{2}}{J(J+1)}-1\right\}\left[\frac{\frac{3}{4} C(C+1)-I(I+1) J(J+1)}{2(2 J+3)(2 J-1) I(2 I-1)}\right],
\end{aligned}
$$

where

$$
\begin{gathered}
C=F(F+1)-I(I+1)-J(J+1), \\
F=J+1, J+I-1 \ldots . \cdot J-1 \mid, \\
e Q q=\text { quadrupole coupling constant. } \\
K=\text { projection of } J \text { on molecular } \\
\text { symmetry axis. }
\end{gathered}
$$

A tabulation of the function in square brackets for $J=0$ to 10 is given in table 3 . The somewhat more complex situation when two nuclei have quadrupole coupling is discussed in reference [51].

The maximum absorption of a spectral line is independent of pressure and may be written [58]

$$
\alpha_{\max .}=\frac{8 \pi^{2} N f\left|\mu_{i j}\right|^{2} \nu^{2}}{3 c k T \Delta \nu}
$$

where $N$ is the number of molecules per cubic centimeter,

$f$ is the fraction of the molecules in the lower state of the transition,

$\nu$ is the frequency of the line,

$c$ is the velocity of light,

$k$ is Boltzmann's constant,

$T$ is the absolute temperature,

$\Delta \nu$ is the half width of the line at half maximum,

$\mu_{i j}$ is the electric dipole matrix element.

The vibrational frequencies and the classical approximation for the sum of rotational states necessary to calculate $f$, as well as expressions for the dipole matrix elements for the various types of molecules may be found in Herzberg [26], or $\mathrm{Wu}$ [61], who also discuss more accurate formulae for the energy levels. The corresponding information for asymmetric molecules is tabulated in the papers of King, Hainer, and Cross [9, 29]. Since the hyperfine splitting is small compared to the 
line frequency, a total absorption coefficient may be calculated ignoring the nuclear interaction, the result being then divided among the hyperfine components. Relative intensities of the hyperfine components can be obtained from tables of relative intensities of the analogous fine structure components $[8,60]$.

Thus the only quantity not as yet evaluated on the right-hand side of the formula above is $\Delta \nu$ the half width of the line at half maximum intensity. Although it surely depends on the dipole moment, among other things, no good theoretical evaluation is possible at present. ${ }^{*}$ It has in a few cases been measured experimentally, however, and in these cases the formula for the intensity given above has been satisfactorily confirmed [53]. At low pressures $p / \Delta \nu$ is constant for a given transition, and for the cases where $\Delta \nu$ has not been measured, we have assumed the reasonable value of $\Delta \nu=25 \mathrm{mc}$ for $1 \mathrm{~mm}$ of mercury. It is this approximation that limits the accuracy of most of the intensities listed in these tables rather than the basic assumptions of the theory or the use of the classical sum of states, except in certain cases where the dipole moments have not been accurately measured. Errors as large as a factor of two or more would not be at all surprising in the cases where the half-widths have not been measured, but we consider that intensities with errors of even this magnitude may be useful, and may be easily corrected when better half-widths become available. The relative intensities for the various isotopes, hyperfine components, and excited states of a single molecule are considerably more reliable.

After substituting the accepted values for the universal constants and putting $e^{-h_{\nu} / k T} \cong 1$, we find for the assumed temperature of $300^{\circ} \mathrm{K}$ and $\Delta \nu=25 \mathrm{mc}$, the intensities of pure rotational spectra become:

For linear molecules

$$
\gamma_{\max .}=2.19 \times 10^{-18} \mu^{2} \nu^{3} \mathrm{~cm}^{-1} .
$$

For symmetric top molecules

$$
\gamma_{\text {max } .}=4.83 \times 10^{-22} \sqrt{A} \mu^{2} \nu^{3}\left\{1-\frac{K^{2}}{(J+1)^{2}}\right\} \mathrm{cm}^{-1},
$$

where

$\mu$ is in Debye units $\left(10^{-18} \mathrm{esu} / \mathrm{cm}\right)$, $\nu$ is the frequency in megacycles,

*A considerable amount of work has been published on this problem since this introduction was written.
$A$ is the reciprocal moment of inertia in megacycles $=10^{-6} h / 8^{2} \pi I_{A}$,

$J$ is the quantum number of total angular momentum,

$K$ is the quantum number of the component of the angular momentum along the figure axis.

In some molecules, nuclear spin and molecular symmetry considerations influence intensities. The only common case involves three identical nuclei of spin $1 / 2$ off the molecular axis as in $\mathrm{CH}_{3} \mathrm{Cl}$ or $\mathrm{AsF}_{3}$. In such molecules, the intensity of each line involving a value of $K$ that is a multiple of three (including zero) is enhanced with respect to other lines by a factor of two.

Throughout the table we have given reciprocal moments of inertia, $B$ and $A$, quadrupole couplings, (eqQ), doubling constants, $q$, and the $\alpha$ 's and $D_{v}$ 's in megacycles per second. The dipole moment, $\mu$, is always in Debye units, and is taken from "Tables of Electric Dipole Moments" by L. G. Wesson, published by Massachusetts Institute of Technology Laboratory for Insulation Research, unless otherwise stated.

The cases of ammonia and $\mathrm{O}_{2}$, and probably some of the spectra not yet analyzed are in one way or another exceptions to the cursory treatment above, and are, where possible, discussed individually.

\section{Symbols Used in Tables}

A, Largest reciprocal moment of inertia for asymmetric molecules, or unique reciprocal moment of inertia for symmetric top molecules, in megacycles.

$\alpha_{i}, \quad$ Change in reciprocal moment of inertia per quantum of excitation of the $i^{\text {th }}$ vibrational state, in megacycles.

$B$ Intermediate moment of inertia for asymmetric top molecules, or nonunique reciprocal moment of inertia for symmetric top molecules, in megacycles.

$B_{0}$, Average $B$ for ground vibrational state, in megacycles.

$C$, Smallest reciprocal moment of inertia for asymmetric top molecules, in megacycles.

$D$, Centrifugal distortion coefficient in megacycles.

$\Delta \nu, \quad$ Half-width of line in megacycles at half maximum (line width parameter) with gas pressure $1 \mathrm{~mm}$ of mercury. 
eqQ, Quadrupole coupling constant, in megacycles.

$F$, Total angular momentum quantum number.

$F_{i}, \quad F$ of initial or lower energy state.

$F_{l i}$, Vector sum of $J$ and spin of nucleus with larger quadrupole coupling, in cases where two nuclei have appreciable quadrupole coupling. Subscript $i$ indicates initial or lower energy state.

$F_{f}, \quad F$ of final or higher energy state.

$F_{l f}$, Same as $F_{l i}$, but for final or higher energy state.

$\gamma$, Maximum absorption coefficient in $\mathrm{cm}^{-1}$, or "intensity."

I, Nuclear spin (in units $h / 2 \pi$ ).

$J, \quad$ Total orbital angular momentum quantum number.

$K$, Projection of $J$ on molecular symmetry axis.

M, Medium intensity $\left(5 \times 10^{-7}-10^{-5} \mathrm{~cm}^{-1}\right)$.

$\mu$, Dipole moment in Debye Units $\left(10^{-18} \mathrm{esu}\right)$.

$q$, l-type doubling constant in megacycles.

$S$, Strong Intensity. $\left(>10^{-5} \mathrm{~cm}^{-1}\right)$.

$v_{i}$, Quantum number of the $i^{\text {th }}$ normal vibration.

$W$ Weak Intensity. $\quad\left(<5 \times 10^{-7} \mathrm{~cm}^{-1}\right)$.

$$
\text { TABLE } 1
$$

\section{Molecules Listed Alphabetically by Chemical Symbol}

$\mathrm{AsF}_{3}$ (Arsenic trifluoride).

The value of $A$ was calculated from the estimates of internuclear angles and distances given by reference !13], and the intensities of the lines for which the transitions have not been assigned were calculated by comparing the observed relative intensities with those of the identified lines. The fraction of molecules in the ground vibrational state was assumed to be 0.9 .

$$
\left.\begin{array}{rl}
\mu & =2.65 \\
B & =5,883.0 \\
A & =3900 . \\
(e q Q)_{\mathrm{A} \mathrm{s}} & =-235
\end{array}\right\} \text { Reference [13]. }
$$

$\mathrm{AsF}_{3}$

$J=1-2, K=0$

$\left.\begin{array}{cccc|c}F_{i} & F_{f} & \text { Frequency } & \text { Intensity } & \begin{array}{c}\text { Refer- } \\ \text { ence }\end{array} \\ 1 / 2 & 3 / 2 & 23,458.6 & 3.1 \times 10^{-7} & \\ 5 / 2 & 5 / 2 & 23,463.0 & 3.4 \times 10^{-7} & \\ 1 / 2 & 1 / 2 & 23,517.0 & 3.1 \times 10^{-7} & \\ 5 / 2 & 7 / 2 & 23,522.4 & 2.3 \times 10^{-6} & \\ 3 / 2 & 5 / 2 & 23] \\ 3 / 2 & 3 / 2 & 23,564.6 & 3.9 \times 10^{-7}\end{array}\right\}$

$J=1-2, K=1$

$$
\left.\begin{array}{llll}
3 / 2 & 5 / 2 & 23,472.6 & 5.8 \times 10^{-7} \\
3 / 2 & 3 / 2 & 23,494.2 & 3.0 \times 10^{-7} \\
5 / 2 & 5 / 2 & 23,501.6 & 2.4 \times 10^{-7} \\
5 / 2 & 7 / 2 & 23,532.1 & 1.1 \times 10^{-6} \\
1 / 2 & 1 / 2 & 23,575.3 & 2.3 \times 10^{-7}
\end{array}\right\}
$$

unidentified (probably excited states]

$$
\left.\begin{array}{rr}
23,512.9 & 1.5 \times 10^{-7} \\
23,543.2 & 3 \times 10^{-7} \\
23,553.0 & 5 \times 10^{-7}
\end{array}\right\}
$$

$\mathrm{BH}_{3} \mathrm{CO}$ (Borine carbonyl).

Vibrational frequencies of $\mathrm{BH}_{3} \mathrm{CO}$ have not been analyzed. The excited vibrational levels for which $B$ and $\mu$ are given here are hence arbitrarily labeled with subscripts 1 and 2 . For calculation of intensities, the fraction of molecules in the ground vibrational state was assumed to be 0.9 .

$$
\begin{array}{rlrl}
\mu & =1.79 & {[49 \mathrm{~d}]} & \\
\mu_{v_{2=1}}=1.77 & {[49 \mathrm{~d}]} & \\
A & \simeq 122,000 \quad[22] & \\
& \mathrm{B}^{10} \mathrm{H}_{3} \mathrm{CO} & \mathrm{B}^{11} \mathrm{H}_{3} \mathrm{CO} \\
\mathrm{B}_{0}= & 8979.4 & 8657.2 \\
B_{v_{1=1}}= & 9002.7 & \\
B_{v_{2}=1} & = & 8985.8 & \\
D_{J} & = & 0.177 & \\
(\text { eq Q) } & = & +3.30 & +1.55
\end{array}
$$

$[22,49 \mathrm{~d}]$

[49d]

[49d]

[49d]

[22]

$$
\mathrm{B}^{10} \mathrm{H}_{3} \mathrm{C}^{12} \mathrm{O}^{16} \quad F_{i} \quad F_{f} \text { Frequency Intensity }
$$$$
\begin{array}{lll}
J=0-1 & 17,961.2 & 7.8 \times 10^{-7}
\end{array}
$$

[49d]

$J=1-2, K=0$

$$
\begin{aligned}
& \text { ( } \\
& \begin{array}{l}
3 \\
3
\end{array} \\
& \begin{array}{l}
2 \\
3 \\
1
\end{array} \\
& 1 \text { ) } \\
& 35,919.53 \quad 4.2 \times 10^{-6} \\
& 5 \\
& 2\} 35,919.86 \quad 5.9 \times 10^{-7} \\
& 4 \\
& 435,920.14 \quad 1.1 \times 10^{-6} \\
& J=1-2, K=1 \\
& \mathrm{~B}^{11} \mathrm{H}_{3} \mathrm{C}^{12} \mathrm{O}^{16} \\
& J=1-2, K=0 \\
& 1 / 2 \\
& 3 / 2 \\
& 5 / 2 \\
& 3 / 2 \\
& J=1-2, K=1 \\
& 5 / 2 \\
& \begin{array}{l}
1 / 2 \\
5 / 2
\end{array} \\
& 5 / 2 \\
& 5 / 2 \\
& \begin{array}{l}
3 / 2 \\
3 / 2
\end{array} \\
& 3 / 2 \\
& \text { 34, 628. } 62 \\
& 34,628.90 \\
& 7 / 2 \\
& 3 / 2\} 34,629.32 \\
& \text { 35, 917. } 62 \\
& 35,917.88 \\
& \text { 4. } 1 \times 10^{-7} \\
& \text { 35, 918. } 20 \\
& \text { 2. } 0 \times 10^{-6} \\
& 37 \\
& 35,918.50 \\
& \text { 4. } 5 \times 10^{-7} \\
& \text { 1. } 3 \times 10^{-6} \\
& 1 / 2 \quad 34,627.24 \quad 1.5 \times 10^{-6} \\
& 7 / 2 \quad 34,627.50 \\
& 5 / 2 \\
& 3 / 2,34,627.73 \\
& \text { 7. } 3 \times 10^{-6} \\
& \text { 3. } 6 \times 10^{-6} \\
& 5 / 2 \quad 34,627.89 \quad 3.8 \times 10^{-6}
\end{aligned}
$$


BrCN (Cyanogen bromide).

The half-width reported in [53] has been used in calculating the intensity.

$$
\begin{aligned}
\mu & =2.94 \\
\Delta \nu & =21 . \pm 3 .[53]
\end{aligned}
$$

$\begin{array}{rlr} & \mathrm{Br}^{79} \mathrm{C}^{12} \mathrm{~N}^{14} & \mathrm{Br}^{81} \mathrm{C}^{12} \mathrm{~N}^{14} \\ \mathrm{~B}_{0} & =4120.190 & 4096.760 \\ (e q Q)_{\mathrm{Br}} & =686.2 & 573.2 \\ { }^{9}(e q Q)_{\mathrm{N}}= & -3.83 & \\ \alpha_{1}= & 11.36 & 11.23 \\ \alpha_{2}=-11.49 & -11.49 \\ q= & 3.91 & 3.845\end{array}$

$\mathrm{Br}^{79} \mathrm{C}^{12} \mathrm{~N}^{14}$

$\mathrm{J}=9-10, v=0$

$\mathrm{Br}^{81} \mathrm{C}^{13} \mathrm{~N}^{14}$

4049. 606

$\mathrm{Br}^{79} \mathrm{C}^{13} \mathrm{~N}^{14}$

4049. 606

$[53,42]$

$\mathrm{Br}^{79} \mathrm{C}^{13} \mathrm{~N}^{14}$

$\left.\begin{array}{lccc}F_{i} & F_{f} & \text { Frequency } & \text { Intensity } \\ 3 / 2 & 3 / 2 & 24,583.00 & 4.3 \times 10^{-6} \\ 5 / 2 & 5 / 2 & 24,633.71 & 5.6 \times 10^{-6} \\ 5 / 2 & 7 / 2 & 24,713.05 & 6.5 \times 10^{-5} \\ 7 / 2 & 9 / 2 & 24,5 & \\ 1 / 2 & 3 / 2 & 24,755.22 & 2.9 \times 10^{-5} \\ 3 / 2 & 5 / 2 & 24.5 \times 10^{-6}\end{array}\right\}$

[53]

$\mathrm{J}=3-4, v=0$

\begin{tabular}{|c|c|c|}
\hline $7 / 2 \quad 9 / 2$ & $32,581.73$ & $10^{-6}$ \\
\hline $9 / 2 \quad 11 / 2$ & & \\
\hline $5 / 2$ & $32,601,46$ & 9. $2 \times 10^{-7}$ \\
\hline
\end{tabular}

$\mathrm{Br}^{81} \mathrm{C}^{12} \mathrm{~N}^{14}$

$\mathrm{J}=2-3, v=0$

$3 / 2 \quad 3 / 2 \quad 24,465.33$

4. $2 \times 10^{-6}$

$5 / 2 \quad 5 / 2 \quad 24,507.38$

5. $4 \times 10^{-6}$

$5 / 2 \quad 7 / 2$

$7 / 2 \quad 9 / 2$

$24,573.86$

6. $3 \times 10^{-5}$

$\begin{array}{ll}5 / 2 & 7 / 2 \\ 7 / 2 & 9 / 2\end{array}$

$1 / 2 \quad 3 / 2$

$3 / 2 \quad 5 / 2 \quad 24,608.92$

2. $7 \times 10^{-5}$

$\begin{array}{lll}1 / 2 & 3 / 2 & 24,687.11\end{array}$

1. $8 \times 10^{-6}$

[53]

$7 / 2 \quad 7 / 2$

24, 717. 19

4. $2 \times 10^{-6}$

$J=2-3, v_{1}=1$

\begin{tabular}{|c|c|c|c|}
\hline $1 / 2$ & $3 / 2$ & \multirow{2}{*}{$24,760.76$} & \multirow{2}{*}{$7.5 \times 10^{-6}$} \\
\hline $7 / 2$ & $9 / 2$ & & \\
\hline $1 / 2$ & $3 / 2$ & \multirow{2}{*}{$24,784.02$} & \multirow{2}{*}{$7.5 \times 10^{-6}$} \\
\hline $7 / 2$ & $9 / 2$ & & \\
\hline $3 / 2$ & $5 / 2$ & \multirow{2}{*}{$24,803.00$} & \multirow{2}{*}{ 6. $9 \times 10^{-6}$} \\
\hline $5 / 2$ & $7 / 2$ & & \\
\hline $3 / 2$ & $5 / 2$ & \multirow{2}{*}{$24,826.70$} & \multirow{2}{*}{ 6. $9 \times 10^{-6}$} \\
\hline $5 / 2$ & $7 / 2$ & & \\
\hline $7 / 2$ & $7 / 2$ & $24,890.0$ & 7. $3 \times 10^{-7}$ \\
\hline $7 / 2$ & $7 / 2$ & $25,006.0$ & 7. $3 \times 10^{-7}$ \\
\hline
\end{tabular}

$\mathrm{J}=2-3, v_{1}=1$
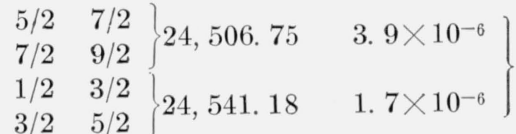

[53]

[53]

$\mathrm{J}=2-3, v_{2}=1$

\begin{tabular}{|c|c|c|c|}
\hline $1 / 2$ & $3 / 2$ & \multirow{2}{*}{$24,6 z 2.93$} & \multirow{2}{*}{ 7. $7 \times 10^{-6}$} \\
\hline $7 / 2$ & $9 / 2$ & & \\
\hline $1 / 2$ & $3 / 2$ & \multirow{2}{*}{$24,645.82$} & \multirow{2}{*}{ 7. $7 \times 10^{-6}$} \\
\hline $7 / 2$ & $9 / 2$ & & \\
\hline $3 / 2$ & $5 / 2$ & \multirow{2}{*}{$24,658.89$} & \multirow[t]{2}{*}{$6.8 \times 10^{-6}$} \\
\hline $5 /$ & 7 & & \\
\hline $\begin{array}{l}3 / 2 \\
5 / 2\end{array}$ & $\begin{array}{l}5 / 2 \\
7 / 2\end{array}$ & $24,682.13$ & 6. $8 \times 10^{-6}$ \\
\hline
\end{tabular}

[53]

$$
\begin{aligned}
& J=2-3, v_{2}=2
\end{aligned}
$$

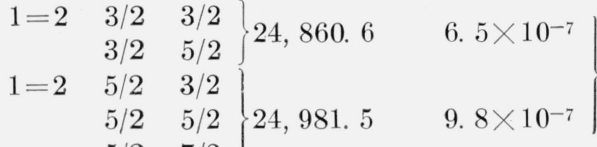

[30]

$\mathrm{J}=3-4, v=0$

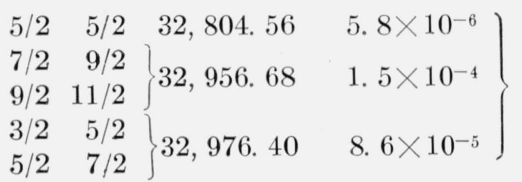

$\mathrm{J}=3-4, v=0$

$\begin{array}{lrr}5 / 2 & 5 / 2 & 32,643.13 \\ 7 / 2 & 7 / 2 & 32,720.28 \\ 7 / 2 & 9 / 2 & 32,770.13 \\ 9 / 2 & 11 / 2 & 32,7 \\ 3 / 2 & 5 / 2 & \\ 5 / 2 & 7 / 2 & 32,786.65 \\ 9 / 2 & 9 / 2 & 3 \varkappa, 913.24\end{array}$

5. $6 \times 10^{-6}$

7. $4 \times 10^{-6}$

1. $4 \times 10^{-4}$

8. $2 \times 10^{-5}$

5. $7 \times 10^{-6}$

$J=8-9, v=0$

73, 742

81, 936

2. $8 \times 10^{-3}$

3. $8 \times 10^{-3}$

[41] 
$\mathrm{B}_{\mathrm{r}}{ }^{81} \mathrm{C}^{13} \mathrm{~N}^{14}$-Continued

\begin{tabular}{|c|c|c|c|c|}
\hline \multirow[t]{4}{*}{$J=3-4, v=0$} & $F_{i}$ & $F_{f}$ & Frequency & Intensity \\
\hline & $7 / 2$ & $9 / 2$ & $(32,392.59$ & 1. $5 \times 10^{-6}$ \\
\hline & $9 / 2$ & $11 / 2$ & & \\
\hline & $\begin{array}{l}3 / 2 \\
5 / 2\end{array}$ & $\begin{array}{l}5 / 2 \\
7 / 2\end{array}$ & $32,409.06$ & 9. $2 \times 10^{-7}$ \\
\hline
\end{tabular}

$\mathrm{CF}_{3} \mathrm{CH}_{3}$. Since the dipole moment and vibrational frequencies of this molecule are not available, it is impossible to calculate the intensity. However, observed intensities are estimated in [5], and these are given. The weaker line is probably due to an excited vibrational state.

$$
\begin{aligned}
B_{0} & =5185 & & {[17] } \\
\mu & =2.33 & & {[39 \mathrm{~b}] }
\end{aligned}
$$

$\mathrm{CF}_{3} \mathrm{CH}_{3}$

$$
\begin{array}{lllr} 
& \text { Frequency } & \text { Intensity } & \\
J=1-2, K=0,1 & 20,679.9 & 5 \times 10^{-7} & {[39 \mathrm{a}]} \\
& 20,710.6 & 1 \times 10^{-6} & {[17,39 \mathrm{a}]} \\
J=2-3 & 20,742.32 & 2 \times 10^{-6} & {[17,39 \mathrm{a}]} \\
& 31,020.7 \\
& 31,066.8 \\
& 31,114.4 & & \\
& & & {[39 \mathrm{a}]}
\end{array}
$$

$\mathrm{CHClF}_{2}$. The spectrum of this asymmetric top molecule has not been analyzed. The assignment of weak, medium, or strong is that of the reference and does not correspond

\begin{tabular}{|c|c|c|c|}
\hline Transition & Frequency & $\begin{array}{l}\text { Observed } \\
\text { intensity }\end{array}$ & \\
\hline \multirow{18}{*}{$8_{3}-8_{4}$} & 21,482 & M & \\
\hline & 21,549 & W & \\
\hline & 21,573 & W & \\
\hline & 21,689 & M & \\
\hline & 21,734 & M & \\
\hline & 22,236 & M & \\
\hline & 22,281 & $\mathrm{~S}$ & \\
\hline & 22,383 & M & \\
\hline & 22,391 & M & \\
\hline & 22,660 & W & \\
\hline & 22,752 & $\mathrm{~S}$ & \\
\hline & 23,181 & M & \\
\hline & 23,206 & W & \\
\hline & 23,214 & M & \\
\hline & 23,220 & M & \\
\hline & 23,234 & W & \\
\hline & 23,323 & M & \\
\hline & 23,361 & M & \\
\hline \multirow[t]{17}{*}{$7_{2}-7_{3}$} & 23,433 & $\mathrm{~S}$ & \\
\hline & 23,649 & M & \\
\hline & 23,770 & M & \\
\hline & 23,812 & M & \\
\hline & 23,986 & M & \\
\hline & 24,021 & M & \\
\hline & 24,150 & W & (excited vibra \\
\hline & 24,294 & M & (excrued viora- \\
\hline & 24,323 & $\mathrm{~S}$ & \\
\hline & 24,352 & M & \\
\hline & 24,357 & W & \\
\hline & 24,450 & $\mathrm{~S}$ & \\
\hline & 24,545 & M & \\
\hline & 24,579 & W & \\
\hline & 24,602 & W & \\
\hline & 24,639 & M & \\
\hline & 24,734 & M & \\
\hline \multirow[t]{3}{*}{$6_{1}-6_{2}$} & 24,770 & $\mathrm{~S}$ & \\
\hline & 24,806 & W & \\
\hline & 24,808 & W & \\
\hline \multirow[t]{4}{*}{$6_{2}-6_{5}$} & 25,248 & M & \\
\hline & 25,350 & $\mathrm{~S}$ & \\
\hline & 25,450 & M & \\
\hline & 25,516 & $\mathrm{~S}$ & \\
\hline \multirow[t]{4}{*}{$5_{0}-5_{1}$} & 25,729 & $\mathrm{~S}$ & \\
\hline & 25,741 & M & \\
\hline & 26,118 & $\mathrm{~S}$ & \\
\hline & 26,163 & M & \\
\hline \multirow[t]{2}{*}{$4_{-1}-4_{0}$} & 26,328 & $\mathrm{~S}$ & \\
\hline & 26,337 & M & \\
\hline $1_{0}-2_{-1}$ & 26,410 & W & \\
\hline $1_{0}-2_{-1}$ & 26,466 & M & \\
\hline
\end{tabular}
necessarily to the convention adopted in this table.

$\left.\begin{array}{cc}\mu=1.29 & \\ \text { Frequency } & \text { Intensity } \\ 22,217 & \text { W } \\ 22,247 & \text { W } \\ 22,305 & \text { M } \\ 22,353 & \text { W } \\ 22,386 & \text { W } \\ 22,410 & \text { S } \\ 22,436 & \text { W } \\ 22,462 & \text { W } \\ 22,481 & \text { M } \\ 22,545 & \text { W } \\ 22,553 & \text { W } \\ 23,308 & \text { S } \\ 23,644 & \text { W } \\ 23,680 & \text { W } \\ 23,733 & \text { S } \\ 23,803 & \text { W } \\ 23,826 & \\ 23,845 & \text { S }\end{array}\right\}$

$\mathrm{CH}_{2} \mathrm{Br}_{2}$ (Methylene bromide)

$$
\begin{aligned}
& \mu \cong 1.6 \\
& \text { Frequency } \\
& 25,056
\end{aligned}
$$

$\mathrm{CH}_{2} \mathrm{CF}_{2}$ (Unsymmetrical diflouroethylene)

$$
\left.\begin{array}{rl}
\mu & =1.366 \\
A_{0} & =11,002 \\
B_{0} & =10,428.5 \\
C_{0} & =5,345.6
\end{array}\right\}[37 \mathrm{a}]
$$

$\mathrm{C}^{12} \mathrm{H}_{2} \mathrm{C}^{12} \mathrm{~F}_{2}$ 


\begin{tabular}{|c|c|c|c|}
\hline Transition & $\begin{array}{c}\text { Frequency } \\
26.634\end{array}$ & $\begin{array}{c}\text { Observed } \\
\text { intensity } \\
\mathrm{S}\end{array}$ & \\
\hline \multirow[t]{4}{*}{$3_{-2}-3_{-1}$} & 26,649 & M & \\
\hline & 26,723 & W & \\
\hline & 26,832 & M & \\
\hline & 26,865 & $\mathrm{~S}$ & \\
\hline $3_{-3}-3_{0}$ & 26,880 & M & \\
\hline $1_{-1}-2_{-2}$ & 26,992 & $\mathrm{~S}$ & \\
\hline \multirow{3}{*}{$4_{-2}-4_{1}$} & 27,014 & $\mathrm{~S}$ & \\
\hline & 27,112 & $\mathrm{M}$ & \\
\hline & 27,216 & M & \\
\hline \multirow[t]{3}{*}{$5_{-1}-5_{2}$} & 27,297 & $\mathrm{~S}$ & \\
\hline & 27,412 & M & \\
\hline & 27,681 & $\mathrm{~S}$ & \\
\hline \multirow[t]{11}{*}{$6_{0}-6_{3}$} & 27,818 & $\mathrm{~S}$ & \\
\hline & 27,972 & $\mathrm{~S}$ & [37a] \\
\hline & 28,174 & $\mathrm{~S}$ & \\
\hline & 28,314 & W & \\
\hline & 28,339 & M & \\
\hline & 28,412 & $\mathrm{~S}$ & \\
\hline & 28,439 & M & \\
\hline & 28,458 & M & \\
\hline & 28,551 & W & \\
\hline & 28,575 & W & \\
\hline & 28,615 & W & \\
\hline \multirow[t]{4}{*}{$7_{1}-7_{4}$} & 28,689 & W & \\
\hline & 28,852 & & \\
\hline & 28,858 & & \\
\hline & 28,954 & $\mathrm{~S}$ & \\
\hline $1_{1}-2_{0}$ & 36,632 & M & \\
\hline
\end{tabular}

$\mathrm{CH}_{2} \mathrm{Cl}_{2}$ (Methylene chloride).

Turner [57] has given the relative intensities listed for this asymmetric top molecule. This author has also made transition assignments but they have been questioned by Gwinn [23a], and are not listed here. Frequencies given are a plausible compromise between those of reference [57] and [11].

$$
\mu=1.6
$$

$\begin{array}{cl}\text { Frequency } & \\ 23,476 & {[11]} \\ 24,440 & {[57]} \\ 24,471 & {[57]} \\ 24,577 & {[57,11]} \\ 24,627 & {[57]} \\ 24,631 & {[57]} \\ 24,636 & {[57,11]} \\ 24,842 & {[57,11]} \\ 24,916 & {[57]} \\ 24,967 & {[57]} \\ 24,970 & {[57]} \\ 24,976 & {[57]} \\ 24,996 & {[11]} \\ 25,018 & {[57]} \\ 25,038 & {[57]} \\ 25,046 & {[57]} \\ 25,047 & {[57]} \\ 25,053 & {[57,11]}\end{array}$

Observed relative intensity

$\left.\begin{array}{clr}25,073 & {[57,11]} & 7 \\ 25,099 & {[57,11]} & 2 \\ 25,123 & {[57,11]} & 8 \\ 25,221 & {[11]} & \\ 25,269 & {[57,11]} & 7 \\ 25,280 & {[57]} & 6 \\ 25,710 & {[57]} & 7 \\ 25,745 & {[57]} & 2 \\ 25,748 & {[57]} & 6 \\ 25,751 & {[57]} & 2 \\ 25,848 & {[57]} & 10 \\ 26,007 & {[57]} & 7 \\ 26,295 & {[57]} & 9 \\ \begin{array}{c}\mathrm{B} \\ \mathrm{Br}\left(\text { Methyl bromide }^{2}\right.\end{array} & \mu=1.79 \\ \mathrm{CH}_{3} \mathrm{Br}^{79} & \mathrm{CH}{ }_{3} \mathrm{Br}^{81} & \\ \mathrm{Br}_{0}=9568.4 & 9532 \\ (\text { eqQ })_{\mathrm{Br}=577.3} & 482.4\end{array}\right\}$ Reference $[23,38 \mathrm{a}]$.

$\mathrm{C}^{12} \mathrm{H}_{3} \mathrm{Br}^{79}$

$$
\left.\begin{array}{rlll}
F_{i} & F_{f} & \text { Frequency } & \text { Intensity } \\
3 / 2 & 1 / 2 & 18,922.41 & 4.8 \times 10^{-7} \\
3 / 2 & 3 / 2 & 19,252.13 & 9.7 \times 10^{-7} \\
3 / 2 & 5 / 2 & 19,107.97 & 1.5 \times 10^{-6}
\end{array}\right\}
$$$$
J=1-2, K=1
$$$$
\left.\begin{array}{llll}
1 / 2 & 1 / 2 & 38,128.40 & 1.4 \times 10^{-6} \\
1 / 2 & 3 / 2 & 38,200.52 & 1.4 \times 10^{-6} \\
5 / 2 & 7 / 2 & 38,237.14 & 7.0 \times 10^{-6} \\
5 / 2 & 5 / 2 & 38,309.45 & 1.6 \times 10^{-6} \\
3 / 2 & 3 / 2 & 38,330.25 & 1.9 \times 10^{-6} \\
3 / 2 & 5 / 2 & 38,381.70 & 3.5 \times 10^{-6}
\end{array}\right\}
$$

$\mathrm{C}^{12} \mathrm{H}_{3} \mathrm{Br}^{31}$

$$
\begin{aligned}
& J=0-1, K=0 \\
& \left.\begin{array}{llll}
3 / 2 & 1 / 2 & 18,943.77 & 4.7 \times 10^{-7} \\
3 / 2 & 3 / 2 & 19,160.82 & 9.5 \times 10^{-7} \\
3 / 2 & 5 / 2 & 19,040.32 & 1.4 \times 10^{-6}
\end{array}\right\} \\
& J=1-2, K=0 \\
& \left.\begin{array}{llll}
3 / 2 & 3 / 2 & 38,030.77 & 2.4 \times 10^{-6} \\
3 / 2 & 5 / 2 & & \\
5 / 2 & 7 / 2 & 38,116.65 & 1.4 \times 10^{-5} \\
1 / 2 & 1 / 2 & 38,126.97 & 1.9 \times 10^{-6} \\
5 / 2 & 5 / 2 & 38,237.14 & 2.1 \times 10^{-6} \\
1 / 2 & 3 / 2 & 38,247.77 & 1.9 \times 10^{-6}
\end{array}\right\} \\
& J=1-2, K=1 \\
& \left.\begin{array}{llll}
1 / 2 & 1 / 2 & 38,006.47 & 1.4 \times 10^{-6} \\
1 / 2 & 3 / 2 & 38,066.72 & 1.4 \times 10^{-6} \\
5 / 2 & 7 / 2 & 38,097.45 & 6.7 \times 10^{-6} \\
5 / 2 & 5 / 2 & 38,157.70 & 1.5 \times 10^{-6} \\
3 / 2 & 3 / 2 & 38,175.08 & 1.8 \times 10^{-6} \\
3 / 2 & 5 / 2 & 38,218.21 & 3.5 \times 10^{-6}
\end{array}\right\}
\end{aligned}
$$


$\mathrm{CH}_{3} \mathrm{CCH}$ (Methyl acetylene).

This molecule, while probably a symmetric top, has ten distinct vibrational frequencies, two or three of which are low enough to be appreciably excited at room temperature, the lowest $\left(\nu_{10}=336 \mathrm{~cm}^{-1}\right)$ to several quanta of vibrational energy. In addition, centrifugal distortion will separate levels of equal $J$ but different $K$. The transitions have not been assigned, but we include the observed relative intensities.

$$
\begin{gathered}
\mu=.75 \\
B \simeq 8,408
\end{gathered}
$$

\section{$\mathrm{CH}_{3} \mathrm{CCH}$}

$\left.\begin{array}{rr} & \begin{array}{c}\text { Relative } \\ \text { intensity }\end{array} \\ \text { Frequency } & 50 \\ 51,260 & 80 \\ 51,264 & 100 \\ 51,266 & 10 \\ 51,296 & 10 \\ 51,313 & 10 \\ 51,314 & 5 \\ 51,318 & 5 \\ 51,319 & 15 \\ 51,334 & 30 \\ 51,363 & 30 \\ 51,410 & 30 \\ 51,416 & 15 \\ 51,418 & 15 \\ 51,419 & 15\end{array}\right\}$

$\mathrm{CH}_{3} \mathrm{Cl}$ (Methyl chloride).

$$
\begin{aligned}
& \mu=1.86 \\
& A \cong 150,000
\end{aligned}
$$

$$
\begin{aligned}
& \mathrm{C}^{12} \mathrm{H}_{3} \mathrm{Cl}^{35} \\
& \quad B_{0}=13,292.89[23] \\
& (e q Q)=-75.33[23,27 \mathrm{~b}] \\
& \mathrm{C}^{13} \mathrm{H}_{3} \mathrm{Cl}^{35} \\
& B_{0}=12,796.2[11]
\end{aligned}
$$$$
\mathrm{C}^{12} \mathrm{H}_{3} \mathrm{Cl}^{37}
$$$$
\text { 13, 088. } 19 \text { [23] }
$$$$
-58.5[23,27 \mathrm{~b}]
$$

$\mathrm{C}^{13} \mathrm{H}_{3} \mathrm{Cl}^{37}$$$
12,590.0[11]
$$

$\mathrm{CH}_{3} \mathrm{CN}$ (Methyl cyanide).

$$
\begin{aligned}
& \mathrm{C}^{13} \mathrm{H}_{3} \mathrm{Cl}^{37} \\
& J=0-1, K=0
\end{aligned}
$$$$
\left.\begin{array}{llll}
3 / 2 & 3 / 2 & 25,577.2 & 4.5 \times 10^{-8} \\
3 / 2 & 5 / 2 & 25,596.0 & 6.7 \times 10^{-8} \\
3 / 2 & 1 / 2 & 25,611.2 & 2.2 \times 10^{-8}
\end{array}\right\}
$$

$$
\left.\begin{array}{llll}
3 / 2 & 3 / 2 & 25,195.2 & 1.4 \times 10^{-9} \\
3 / 2 & 5 / 2 & 26,183.0 & 2.1 \times 10^{-9} \\
3 / 2 & 1 / 2 & 25,167.4 & 6.9 \times 10^{-10}
\end{array}\right\}
$$

$$
\begin{aligned}
\mu & \simeq 3.4 \\
(e q Q)_{N} & =-4.67 \\
A & \simeq 150,000 \\
B_{0} & =9198.45
\end{aligned}
$$

$$
\left.\begin{array}{crrrcc} 
& F_{i} & F_{f} & K & \text { Frequency } & \text { Intensity } \\
J=0-1 & 1 & 1 & 0 & 18,400-1.40 & 4.7 \times 10^{-6} \\
& 1 & 2 & 0 & 18,400 & 7.8 \times 10^{-6} \\
& 1 & 0 & 0 & 18,400+2.10 & 1.6 \times 10^{-6}
\end{array}\right\}
$$

Excited vibrational

states

$\mathrm{CH}_{3} \mathrm{~F}$ (Methyl fluoride)

$$
\begin{aligned}
& \mu=1.81 \\
& A \cong 150,000 \\
& \Delta \nu=20 \text {. Reference [19] } \\
& \mathrm{C}^{12} \mathrm{H}_{3} \mathrm{~F} \quad \mathrm{C}^{13} \mathrm{H}_{3} \mathrm{~F} \\
& B_{0}=25,535.85 \quad 24,862.37
\end{aligned}
$$

$\begin{array}{lcc}\mathrm{C}^{12} \mathrm{H}_{3} \mathrm{~F} & \text { Frequency } & \text { Intensity } \\ J=0-1, K=0 & 51,071.69 & 1.4 \times 10^{-4}\end{array}$

$\mathrm{C}^{13} \mathrm{H}_{3} \mathrm{~F}$

$J=0-1, K=0 \quad 49,724.73 \quad 1.5 \times 10^{-6}$

$\mathrm{CH}_{3} \mathrm{I}$ (Methyl iodide).

$$
\begin{aligned}
& \mu=1.5 \\
& A \cong 150,000 \\
& (e q Q)_{1}=-1934 \\
& B_{0}=\mathrm{C}^{12} \mathrm{H}_{3} \mathrm{I} \quad \mathrm{C}^{13} \mathrm{H}_{3} \mathrm{I} \\
& 7501.250 \quad 7119.040
\end{aligned}
$$


$\mathrm{C}^{12} \mathrm{H}_{3} \mathrm{I}$

$$
J=1-2, K=0 \begin{array}{cccc}
F_{i} & F_{f} & \text { Frequency } & \text { Intensity } \\
3 / 2 & 5 / 2 & 29,598.95 & 9.2 \times 10^{-7} \\
7 / 2 & 7 / 2 & 29,673.95 & 1.4 \times 10^{-6} \\
7 / 2 & 5 / 2 & 29,773.95 & 2.3 \times 10^{-7} \\
3 / 2 & 3 / 2 & 29,872.52 & 1.4 \times 10^{-6} \\
7 / 2 & 9 / 2 & 30,046.99 & 5.0 \times 10^{-6} \\
5 / 2 & 7 / 2 & 30,079.72 & 2.6 \times 10^{-6} \\
3 / 2 & 1 / 2 & 30,121.32 & 1.0 \times 10^{-6} \\
5 / 2 & 5 / 2 & 30,179.71 & 2.6 \times 10^{-6} \\
5 / 2 & 3 / 2 & 30,453.46 & 6.3 \times 10^{-7}
\end{array}
$$$$
J=1-2, K=1
$$$$
\left.\begin{array}{llll}
5 / 2 & 7 / 2 & 29,735.71 & 2.5 \times 10^{-6} \\
5 / 2 & 5 / 2 & 29,782.71 & 1.8 \times 10^{-6} \\
5 / 2 & 3 / 2 & 29,923.50 & 1.8 \times 10^{-6} \\
7 / 2 & 7 / 2 & 29,939.87 & 1.4 \times 10^{-6} \\
7 / 2 & 5 / 2 & 29,986.84 & 2.4 \times 10^{-7} \\
3 / 2 & 5 / 2 & 30,075.08 & 9.5 \times 10^{-7} \\
7 / 2 & 9 / 2 & 30,123.64 & 5.1 \times 10^{-6} \\
3 / 2 & 3 / 2 & 30,215.95 & 1.4 \times 10^{-6}
\end{array}\right\}
$$
${ }^{12} \mathrm{H}_{3} \mathrm{~N}^{14} \mathrm{C}^{12} \mathrm{~S}^{32}$

$$
J=1-2, K=0
$$

$\left.\begin{array}{llll}3 / 2 & 5 / 2 & 28,069.99 & 1.0 \times 10^{-8} \\ 7 / 2 & 7 / 2 & 28,145.01 & 1.5 \times 10^{-8} \\ 3 / 2 & 3 / 2 & 28,343.64 & 1.5 \times 10^{-8} \\ 7 / 2 & 9 / 2 & 28,518.14 & 5.5 \times 10^{-8} \\ 5 / 2 & 7 / 2 & 28,550.86 & 2.9 \times 10^{-8} \\ 5 / 2 & 5 / 2 & 28,650.91 & 2.9 \times 10^{-8}\end{array}\right\}$

$J=1-2, K=1$

$$
\left.\begin{array}{llll}
5 / 2 & 7 / 2 & 28,206.90 & 2.8 \times 10^{-8} \\
5 / 2 & 5 / 2 & 28,253.84 & 2.0 \times 10^{-8} \\
7 / 2 & 7 / 2 & 28,411.19 & 1.5 \times 10^{-8} \\
7 / 2 & 9 / 2 & 28,594.74 & 5.6 \times 10^{-8} \\
3 / 2 & 3 / 2 & 28,687.21 & 1.5 \times 10^{-8}
\end{array}\right\}
$$

$\mathrm{CH}_{3} \mathrm{NC}$ (Methyl isocyanide).

$$
\left.\begin{array}{rll}
(e q Q)_{N}<0.5, A \simeq & 150,000 \\
B_{0}= & \frac{\mathrm{C}^{12} \mathrm{H}_{3} \mathrm{NC}^{12}}{10,052.79} & \frac{\mathrm{C}^{12} \mathrm{H}_{3} \mathrm{NC}^{13}}{9,695.802}
\end{array}\right\}
$$$$
\mathrm{C}^{12} \mathrm{H}_{3} \mathrm{~N}^{14} \mathrm{C}^{12}
$$

$J=0-1, K=0$

Frequency

$J=1-2, K=1$

20,160

$J=1-2, K=0$

Excited vibrational states

40, 210. 27

40, 211. 16

40, 313. 37

40, 364. 07

$40,366.55$

40, 424. 49

38, 782. 21

38, 783. 21

\section{$\mathrm{C}^{12} \mathrm{H}_{3} \mathrm{~N}^{14} \mathrm{C}^{13}$}

$J=1-2, K=1$

$J=1-2, K=0$
[23]

$$
J=3-4
$$

[23]

$$
J=4-5
$$

$\begin{array}{rlr}\mu= & 3.18 & \\ & \mathrm{CH}_{3} \mathrm{NCS}^{32} & \mathrm{CH}_{3} \mathrm{NCS}^{34} \\ 1 / 2(B+C)= & 2526.2 & 2461.7\end{array}$

[1a]

Observed

relative

Frequency intensity

20. 020

$3_{-2}-4_{-3} 20,140$

Deg. line $\quad 20,216$

20,230

Deg. line, E 20, 241

Deg. line, D 20, 350

20,413

Deg. line, J 20, 443

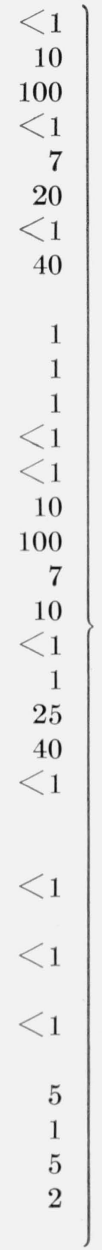

[36]

$\mathrm{C}^{13} \mathrm{H}_{3} \mathrm{~N}^{14} \mathrm{C}^{12} \mathrm{~S}^{3}$

\begin{tabular}{llrr}
$J=4-5$ & Deg. line & 24,143 & $<1$ \\
$\begin{array}{l}\mathrm{C}^{12} \mathrm{H}_{3} \mathrm{~N}^{14} \mathrm{C}^{12} \mathrm{~S}^{32} \\
J=4-5\end{array}$ & Deg. line & 25,002 & $<1$ \\
$\mathrm{C}^{12} \mathrm{H}_{3} \mathrm{~N}^{14} \mathrm{C}^{12} \mathrm{~S}^{32}$ & & & \\
$J=4-5$ & Deg. line & 24,930 & $<1$ \\
$\mathrm{C}^{12} \mathrm{H}_{3} \mathrm{~N}^{14} \mathrm{C}^{12} \mathrm{~S}^{34}$ & & & \\
$J=3-4$ & Deg. line & 19,700 & 5 \\
& Deg. line, J & 19,929 & 1 \\
\multicolumn{1}{|c}{$4-5$} & Deg. line & 24,609 & 5 \\
\multicolumn{1}{c}{$5-6$} & Deg. line, J & 24,910 & 2 \\
& Deg. line & 29,700 &
\end{tabular}

$\mathrm{CH}_{3} \mathrm{NH}_{2}$ (Methylamine).

$\mu=1.2$
$1 / 2(B+C) \cong 22,700 \quad[18]$
Frequency
21,712
21,935
22,258
22,535
22,590
22,595
22,612
22,732
22,740
22,977
23,115
23,205

24,824

24, 971

$4_{-3}-5_{-4} 25,195$

Deg. line $\quad 25,269$

Deg. line, E 25, 306

$4_{-2}-5_{-3} \quad 25,333$

25,390

25,409

Deg. line, D 25, 442

Deg. line, J 25, 550

$4_{-2}-5_{-3}$, J 25,653

40

$\mathrm{CH}_{3} \mathrm{NCS}$ (Methyl Isothiocyanate). All of the transitions of this slightly asymmetric rotor are approximately degenerate except those for which $\tau=1-J$ and $2-J$. Excited vibrational states are labeled $\mathrm{D}, \mathrm{E}$, and $\mathrm{J}$ in conformity with [19].

\section{Molecular Microwave Spectra Tables}




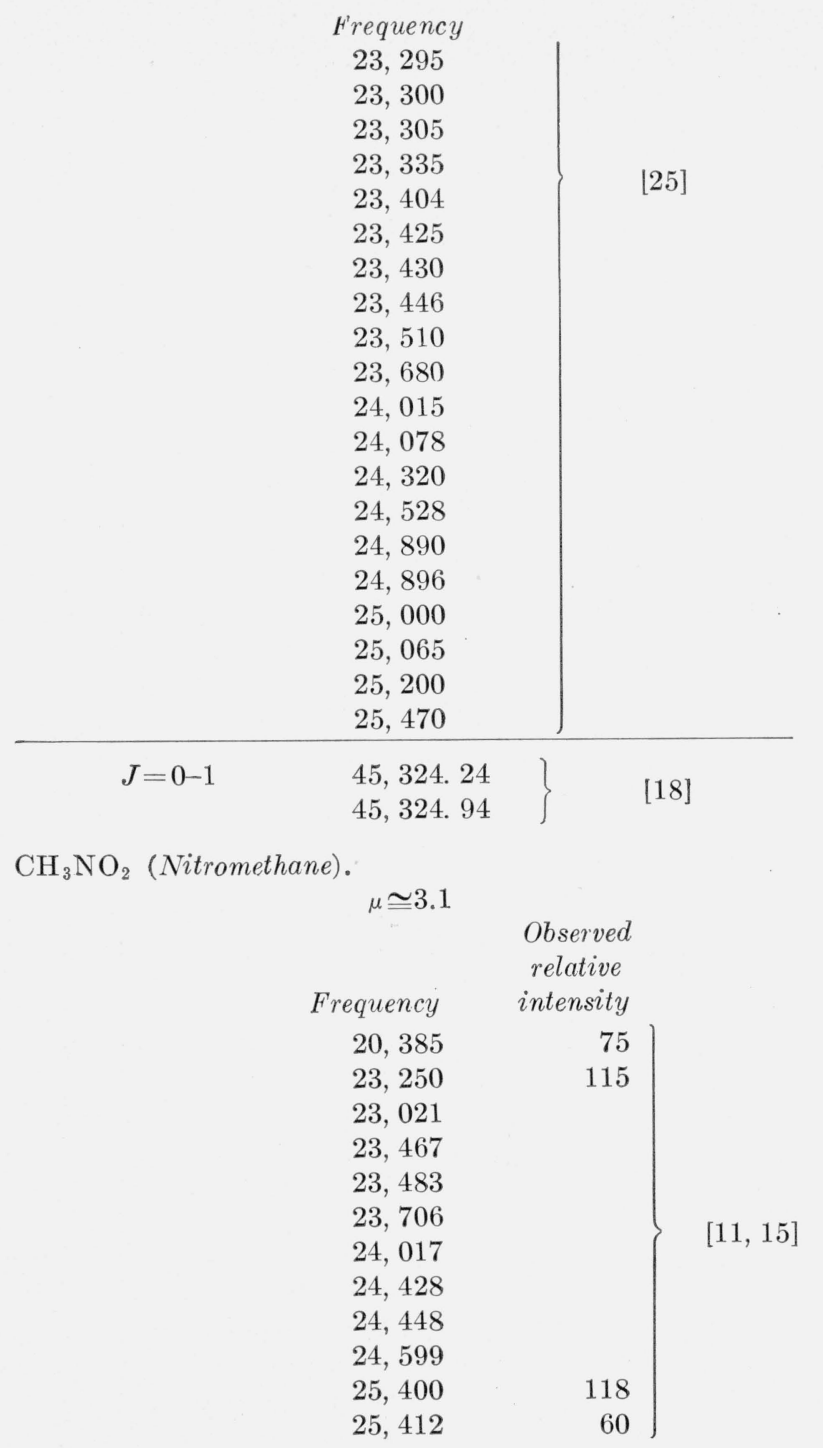

$\mathrm{CH}_{3} \mathrm{OH}$. This asymmetric top molecule has been investigated by five different laboratories. The listed lines repre. sent a reasonable compromise between their somewhat conflicting results. The lines for which a single $J$ is listed are probably due to a hindered internal rotation and are discussed in reference [6].

$$
\begin{aligned}
& \mu \cong 1.66 \\
& J \quad \text { Frequency } \\
& 20,910 \\
& 20,971 \\
& 21,350 \\
& 21,550 \\
& 22,305 \\
& 23,121.2 \\
& 23,346.8 \\
& 23,425 \\
& 23,854.4
\end{aligned}
$$

$[14,25,33]$

$[14,25]$

$[14,25,33]$

$[14,25,33]$

26,120

26,310

26,562

47,840

48,010

48,357

48,363

48, 396

48,404

48, 700

[14, 33]

$\mathrm{CH}_{3} \mathrm{OD}$

$$
\left.\begin{array}{cr} 
& \text { Frequency } \\
J=0-1 & 47,052 \\
& 47,266 \\
& 47,346
\end{array}\right\}
$$

$\mathrm{CH}_{3} \mathrm{SCN}$ (Methyl thiocyanate). Only the strongest lines of this slightly asymmetric molecule have been measured.

$$
\begin{aligned}
& \begin{aligned}
\mu & =3.16 \\
1 / 2(B+C) & =2877 .
\end{aligned} \\
& \mathrm{C}^{12} \mathrm{H}_{3} \mathrm{~S}^{32} \mathrm{C}^{12} \mathrm{~N}^{14} \quad \text { Frequency } \\
& J=3-4 \\
& J=4-5 \\
& \left.\begin{array}{l}
22,680 \\
28,380
\end{array}\right\}
\end{aligned}
$$

$\mathrm{C}_{2} \mathrm{H}_{3} \mathrm{Cl}$ (Vinyl chloride)

$$
\mu=1.44
$$

$$
\begin{aligned}
& \mathrm{C}_{2} \mathrm{H}_{3} \mathrm{Cl}^{35} \\
& \mathrm{~A}_{0}=56,100 \\
& \mathrm{~B}_{0}=6030.5 \\
& \mathrm{C}_{0}=5445.2
\end{aligned}
$$$$
e Q\left(\partial^{2} V / \partial a^{2}\right)_{\mathrm{Cl}}=-57.4
$$$$
e Q\left(\partial^{2} V / \partial b^{2}\right)_{\mathrm{Cl}}=26.2
$$

$\mathrm{C}_{2}^{12} \mathrm{H}_{3} \mathrm{Cl}^{35}$

$\mathrm{C}_{2}^{12} \mathrm{H}_{3} \mathrm{Cl}^{37}$

$$
\begin{gathered}
\text { Transition } \\
1_{01}-2_{02} \\
1_{10}-2_{11} \\
1_{11}-2_{12} \\
1_{01}-2_{02} \\
1_{10}-2_{11} \\
1_{11}-2_{12}
\end{gathered}
$$

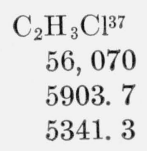

[20a]

[20a] $23,538.9$ $22,369.6$ $22,485.9$ 23, 055. 0 $21,930.2$ 
$\mathrm{C}_{2} \mathrm{H}_{4} \mathrm{O}$ (Ethylene oxide). This asymmetric top has been investigated in two laboratories. Frequencies given are those of reference [10] and [10a] which appear to be the more precise. The lines $\mathrm{C}_{2} \mathrm{D}_{4} \mathrm{O}^{16}$ were observed with enriched material, not with the intensities listed for the deuterium natural abundance of 0.02 percent.

$$
\mu=1.88
$$

$\begin{array}{cc}\mathrm{C}_{2} \mathrm{H}_{4} \mathrm{O} & \mathrm{C}_{2} \mathrm{D}_{4} \mathrm{O} \\ A_{0}=25,471 & 20,389 \\ B_{0}=22,110 & 15,449 \\ C_{0}=14,091 & 11,538\end{array}$

$[10,10 \mathrm{a}]$

Transition Frequency Intensity

$\left.\begin{array}{clll}\mathrm{C}^{12} \mathrm{H}_{4} \mathrm{O}^{16} & 3_{1}-3_{3} & 23,134 & 3.9 \times 10^{-6} \\ & 3_{-1}-3_{1} & 23,610 & 4.1 \times 10^{-6} \\ 4_{0}-4_{2} & 24,834.3 & 1.2 \times 10^{-5} \\ 2_{-2}-2_{0} & 24,924.4 & 3.9 \times 10^{-6} \\ 5_{1}-5_{3} & 29,688 & 1.2 \times 10^{-5} \\ 4_{2}-4_{4} & 34,150 & 1.3 \times 10^{-5} \\ 2_{-1}-2_{1} & 34,158 & 3.0 \times 10^{-6} \\ 6_{0}-6_{2} & 36,791 & 3.4 \times 10^{-5} \\ 7_{1}-7_{3} & 37,329 & 2.5 \times 10^{-5} \\ 5_{-1}-5_{1} & 37,781 & 1.7 \times 10^{-5} \\ 6_{2}-6_{4} & 38,702 & 3.4 \times 10^{-5} \\ 0_{0}-1_{0} & 39,582 & 8.3 \times 10^{-6} \\ 3_{0}-3_{2} & 39,677 & 1.2 \times 10^{-5} \\ 4_{-2}-4_{0} & 41,581 & 1.9 \times 10^{-5} \\ 8_{2}-8_{4} & 43,398 & 6.6 \times 10^{-5} \\ & & \\ \mathrm{C}^{12} \mathrm{D}_{4} \mathrm{O}^{16} & & 7.4 \times 10^{-21} \\ 4_{-2}-4_{0} & 21,964 & 3.4 \times 10^{-21} \\ 3_{-3}-3_{1} & 24,055 & 1.1 \times 10^{-20} \\ 5_{-1}-5_{1} & 24,668 & 2.4 \times 10^{-21} \\ 2_{-1}-2_{1} & 26,365 & 1.8 \times 10^{-20} \\ 6_{-2}-6_{0} & 28,495 & 5.0 \times 10^{-21} \\ 3_{1}-3_{1} & 29,080 & 5.1 \times 10^{-21} \\ 0_{0}-1_{0} & 31,943 & 6.4 \times 10^{-21} \\ 3_{-2}-3_{0} & 33,285 & 3.7 \times 10^{-20} \\ 8_{-2}-8_{0} & 35,068 & 7.4 \times 10^{-21} \\ 3_{0}-3_{2} & 35,341 & 1.8 \times 10^{-20}\end{array}\right\}$

CICN (Cyanogen chloride)

$\begin{aligned} \mu & =2.66 & & {[53] } \\ \Delta \nu & =25 & & {[53] }\end{aligned}$

$[10,39]$

$[20,39]$

$[10,39]$

$[10,39]$

[10]

[10a]

[10a]

[10]

[10]

[10]

[10]

[10]

[10]

[10a]

[10a]$$
[10 \mathrm{a}]
$$

$\mathrm{Cl}^{35} \mathrm{C}^{12} \mathrm{~N}^{14}$

$$
F_{l i} \quad F_{i} \quad F_{l f} \quad F_{f} \quad \text { Frequency Intensity }
$$

\begin{tabular}{|c|c|c|c|c|}
\hline$/ 2$ & $3 / 2$ & & $23,862.57$ & 5. \\
\hline$/ 2 \quad 3 / 2$ & $5 / 2$ & $5 / 2$ & $23,863.5$ & $3.6 \times 10^{-7}$ \\
\hline $\begin{array}{ll}2 & 7 / 2\end{array}$ & $5 / 2$ & $5 / 2$ & $23,863.8$ & 3. $6 \times 10^{-7}$ \\
\hline $3 / 2$ & $5 / 2$ & $3 / 2$ & $23,864.0$ & 1. $9 \times 10^{-6}$ \\
\hline $7 / 2$ & $5 / 2$ & $7 / 2$ & $23,864.2$ & 4. $1 \times 10^{-6}$ \\
\hline $5 / 2$ & $5 / 2$ & $5 / 2$ & $3,864.5$ & 2. $6 \times 10^{-6}$ \\
\hline $5 / 2$ & $5 / 2$ & $7 / 2$ & 64. 9 & 3. $6 \times 10^{-7}$ \\
\hline $5 / 2$ & $3 / 2$ & $3 / 2$ & & \\
\hline $5 / 2$ & $3 / 2$ & $1 / 2$ & & \\
\hline $3 / 2$ & $3 / 2$ & $3 / 2$ & & $5.5 \times 10^{-7}$ \\
\hline $3 / 2$ & $3 / 2$ & $5 / 2$ & 810.0 & \\
\hline $7 / 2$ & $3 / 2$ & $5 / 2$ & $23,878.9$ & $5.0 \times 10^{-7}$ \\
\hline $5 / 2$ & $3 / 2$ & $3 / 2$ & & \\
\hline $5 / 2$ & $3 / 2$ & $5 / 2$ & & \\
\hline & $1 / 2$ & & 3. 30 & 9. $2 \times 10^{-8}$ \\
\hline $7 / 2$ & $7 / 2$ & $7 / 2$ & 23,884 & 7. $7 \times 10^{-\theta}$ \\
\hline $3 / 2$ & $5 / 2$ & $5 / 2$ & & \\
\hline $3 / 2$ & $5 / 2$ & $3 / 2$ & $23,884.8$ & 1. $2 \times 10^{-6}$ \\
\hline $5 / 2$ & $5 / 2$ & $5 / 2$ & $23,884.9$ & 1. $5 \times 10^{-5}$ \\
\hline $5 / 2$ & $7 / 2$ & $7 / 2$ & & \\
\hline $7 / 2$ & $7 / 2$ & $9 / 2$ & $23,885.16$ & 2. $9 \times 10^{-5}$ \\
\hline $3 / 2$ & $7 /$ & $5 / 2$ & & \\
\hline $5 / 2$ & $5 / 2$ & $7 / 2$ & & \\
\hline $1 / 2$ & $5 / 2$ & $3 / 2$ & 23,886 . & 4. $0 \times 10^{-6}$ \\
\hline $5 / 2$ & $7 / 2$ & $5 / 2$ & $23,886.2$ & 1. $2 \times 10^{-\theta}$ \\
\hline $5 / 2$ & $7 / 2$ & $1 / 2$ & & \\
\hline $3 / 2$ & $3 / 2$ & $3 / 2$ & $23,899.59$ & 3. $9 \times 10^{-6}$ \\
\hline $3 / 2$ & $3 / 2$ & $5 / 2$ & & \\
\hline $\begin{array}{l}5 / 2 \\
5 / 2\end{array}$ & $3 / 2$ & & $23,900.20$ & $9.6 \times 10^{-}$ \\
\hline $\begin{array}{l}5 / 2 \\
1 / 2\end{array}$ & $3 / 2$ & $1 / 2$ & & \\
\hline $1 / 2$ & $3 / 2$ & $3 / 2$ & 90 & 2. $1 \times 10$ \\
\hline & $1 / 2$ & & $23,920.9$ & 1. $9 \times 10^{-6}$ \\
\hline
\end{tabular}

$J=1-2, v=0$

$$
5 / 2
$$

$$
5 / 2
$$$$
5 / 2
$$$$
\begin{array}{llll}
5 / 2 & 5 / 2 & 5 / 2 & 5 / 2
\end{array}
$$$$
\begin{array}{llll}
5 / 2 & 5 / 2 & 5 / 2 & 7 / 2
\end{array}
$$$$
\begin{array}{llll}
5 / 2 & 5 / 2 & 3 / 2 & 3 / 2 \\
5 / 2 & 5 / 2 & 3 / 2 & 1 / 2
\end{array}
$$$$
\begin{array}{llll}
5 / 2 & 3 / 2 & 3 / 2 & 3 / 2
\end{array}
$$$$
\begin{array}{llll}
5 / 2 & 3 / 2 & 3 / 2 & 5 / 2
\end{array}
$$$$
\begin{array}{llll}
5 / 2 & 7 / 2 & 3 / 2 & 5 / 2 \\
5 / 2 & 5 / 2 & 3 / 2 & 3 / 2
\end{array}
$$$$
\begin{array}{llll}
5 / 2 & 5 / 2 & 3 / 2 & 5 / 2
\end{array}
$$$$
\begin{array}{llll}
1 / 2 & & 1 / 2 & \\
5 / 2 & 7 / 2 & 7 / 2 & 7 / 2
\end{array}
$$$$
\begin{array}{llll}
3 / 2 & 3 / 2 & 5 / 2 & 5 / 2
\end{array}
$$$$
\begin{array}{llll}
3 / 2 & 3 / 2 & 5 / 2 & 3 / 2 \\
3 / 2 & 5 / 2 & 5 / 2 & 5 / 2
\end{array}
$$$$
\begin{array}{llll}
5 / 2 & 5 / 2 & 7 / 2 & 7 / 2
\end{array}
$$$$
\begin{array}{llll}
5 / 2 & 7 / 2 & 7 / 2 & 9 / 2
\end{array}
$$$$
\begin{array}{llll}
5 / 2 & 3 / 2 & 7 / 2 & 5 / 2
\end{array}
$$$$
\begin{array}{llll}
3 / 2 & 5 / 2 & 5 / 2 & 7 / 2
\end{array}
$$$$
\begin{array}{llll}
3 / 2 & 1 / 2 & 5 / 2 & 3 / 2 \\
5 / 2 & 5 / 2 & 7 / 2 & 5 / 2
\end{array}
$$$$
\begin{array}{llll}
5 / 2 & 5 / 2 & 7 / 2 & 1 / 2
\end{array}
$$$$
\left.\begin{array}{llll}
3 / 2 & 3 / 2 & 3 / 2 & 3 / 2 \\
3 / 2 & 3 / 2 & 3 / 2 & 5 / 2
\end{array}\right\}
$$$$
\begin{array}{lll}
3 / 2 & 5 / 2 & 3 / 2
\end{array}
$$$$
\begin{array}{lll}
3 / 2 & 5 / 2 & 3 / 2
\end{array}
$$$$
\begin{array}{llll}
3 / 2 & 1 / 2 & 3 / 2 & 1 / 2 \\
3 / 2 & 1 / 2 & 3 / 2 & 3 / 2 \\
3 / 2 & & 1 / 2 &
\end{array}
$$$$
J=1-2, v_{2}=1
$$$$
\left.\begin{array}{lllll}
v_{2}=1 & & & 23,917.9 & 4.5 \times 10^{-6} \\
3 / 2 & 5 / 2 & 1_{1} & 23,925.5 & 1.8 \times 10^{-6} \\
3 / 2 & 3 / 2 & 1_{1} & 23,925.7 & 1.4 \times 10^{-6} \\
5 / 2 & 5 / 2 & 1_{1} & 23,928.7 \\
5 / 2 & 7 / 2 & 1_{1} & 23,938.6 & 8.2 \times 10^{-6} \\
1 / 2 & 3 / 2 & 1_{1} & 23,944.4 & 1.7 \times 10^{-6} \\
3 / 2 & 5 / 2 & 1_{2} & 23,948.2 & 4.5 \times 10^{-6} \\
1 / 2 & 1 / 2 & 1_{1} & 23,954.5 & 3.5 \times 10^{-6} \\
3 / 2 & 3 / 2 & 1_{2} & & \\
5 / 2 & 5 / 2 & 1_{2} & 23,958.4 & 1.8 \times 10^{-6} \\
5 / 2 & 7 / 2 & 1_{2} & 23,968.6 & 8.2 \times 10^{-6} \\
1 / 2 & 3 / 2 & 1_{2} & 23,974.4 & 1.7 \times 10^{-6} \\
1 / 2 & 1 / 2 & 1_{2} & 23,984.6 & 1.7 \times 10^{-6}
\end{array}\right)
$$

\section{$[10 a]$}

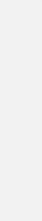

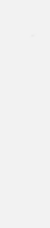

$\left.\begin{array}{llll}5 / 2 & 1_{1} & 23,917.9 & 4.5 \times 10^{-6} \\ 3 / 2 & 1_{1} & 23,925.5 & 1.8 \times 10^{-6} \\ 5 / 2 & 1_{1} & 23,928.7 & 1.4 \times 10^{-6} \\ 7 / 2 & 1_{1} & 23,938.6 & 8.2 \times 10^{-6} \\ 3 / 2 & 1_{1} & 23,944.4 & 1.7 \times 10^{-6} \\ 5 / 2 & 1_{2} & 23,948.2 & 4.5 \times 10^{-6} \\ 1 / 2 & 1_{1} & 23,954.5 & 3.5 \times 10^{-6} \\ 3 / 2 & 1_{2} & & \\ 5 / 2 & 1_{2} & 23,958.4 & 1.8 \times 10^{-6} \\ 7 / 2 & 1_{2} & 23,968.6 & 8.2 \times 10^{-6} \\ 3 / 2 & 1_{2} & 23,974.4 & 1.7 \times 10^{-6} \\ 1 / 2 & 1_{2} & 23,984.6 & 1.7 \times 10^{-6}\end{array}\right)$

$$
\begin{aligned}
& \mathrm{Cl}^{35} \mathrm{C}^{12} \mathrm{~N}^{14} \quad \mathrm{Cl}^{37} \mathrm{C}^{12} \mathrm{~N}^{14} \quad \mathrm{Cl}^{35} \mathrm{C}^{13} \mathrm{~N}^{14} \quad \mathrm{Cl}^{37} \mathrm{C}^{13} \mathrm{~N}^{14} \\
& \left.\mathrm{~B}_{0}=5970.821 \quad 5847.252 \quad 5939.795 \quad 5814.710\right\}[42,53] \\
& \begin{array}{ccc}
(e q Q)_{\mathrm{Cl}}= & -83.4 & -65.3 \\
(e q Q)_{N}= & -3.63
\end{array}
\end{aligned}
$$




$\left.\begin{array}{rr}J=2-3, v=0 & \\ F_{i} & F_{f} \\ 7 / 2 & 7 / 2 \\ 1 / 2 & 3 / 2 \\ 3 / 2 & 5 / 2 \\ 5 / 2 & 7 / 2 \\ 7 / 2 & 9 / 2 \\ 5 / 2 & 5 / 2\end{array}\right\}$

$\mathrm{Cl}^{35} \mathrm{C}^{13} \mathrm{~N}^{14}$

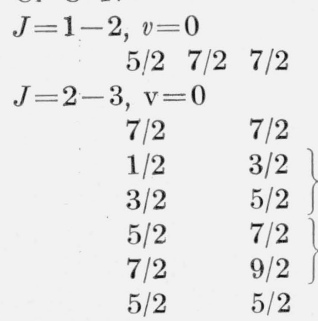

$\mathrm{Cl}^{37} \mathrm{C}^{12} \mathrm{~N}^{14}$

$J=1-2, v=0$

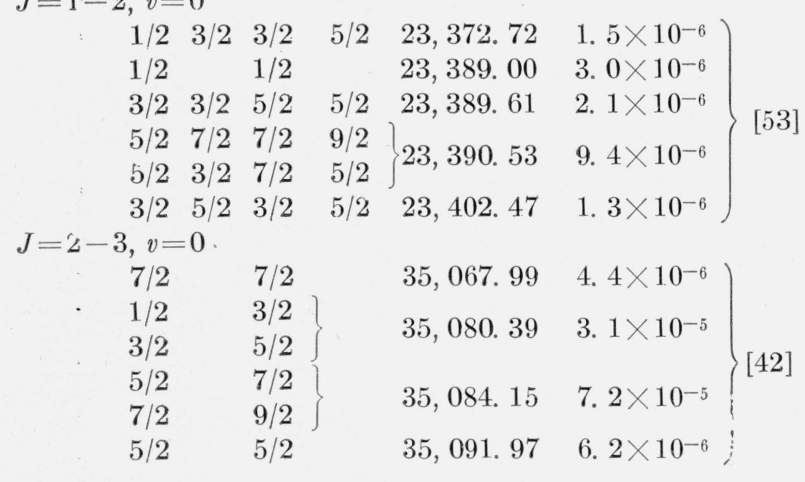

$\mathrm{Cl}^{37} \mathrm{C}^{13} \mathrm{~N}^{14}$

$J=1-2, v=0$

$5 / 2 \quad 7 / 2 \quad 7 / 2 \quad 9 / 2 \quad 23,260.3 \quad 1 \times 10^{-7}$

$J=2-3, v=0$

$$
7 / 2 \quad 9 / 2 \quad 34,889.05 \quad 4.5 \times 10^{-7}
$$

[42]

$\mathrm{HCF}_{3}$ (Fluoroform). In the absence of other information, $A$ was calculated using the $C-F$ distance estimated by the authors, assuming tetrahedral angles. It was also assumed that 0.9 of the molecules were in the ground vibrational state.
$\mathrm{HC}^{12} \mathrm{~F}_{3}$

$$
\left.\begin{array}{rl}
\Delta \nu & =18 \\
\mu & =1.59 \\
B_{0} & =10,348.74 \\
A & =5,600
\end{array}\right\}
$$

$$
J=1-2, K=0,1,2 \quad \text { Frequency Intensity }
$$$$
\text { 41, 394. } 95 \quad 2.2 \times 10^{-5}
$$

$\mathrm{HCN}$ (Hydrocyanic acid). This line is really a triplet, and the calculated intensity is for the sum of all three hyperfine components.

$$
\left.\begin{array}{rl}
\mu & =2.8 \\
B_{0} & =44,336 \\
(e q Q)_{N} & =-4.7 \\
\Delta \nu & =25 .
\end{array}\right\}[41]
$$

$$
\begin{aligned}
& \mathrm{HC}^{12} \mathrm{~N}^{14} \\
& \begin{array}{lll}
J=O-1 & 88,671 & 1.1 \times 10^{-2}
\end{array} \\
& \mathrm{H}_{2} \mathrm{CO} \text { (Formaldehyde) } \\
& \left.\begin{array}{c}
\mu=2.3 \\
A_{0}=364,500 \\
B_{0}=51,720 \\
C_{0}=45,338
\end{array}\right\} \\
& \text { Transition Frequency } \\
& \mathrm{H}_{2} \mathrm{C}^{12} \mathrm{O}^{16} \quad 3_{-1}-3_{-2} \quad 28,974.85 \\
& 4_{-2}-4_{-3} \quad 48,284.60 \\
& 5_{-3}-5_{-4} \quad 72,409.35 \\
& 0_{0}-1_{-1} \quad 72,838.14
\end{aligned}
$$

\begin{tabular}{|c|c|c|c|c|c|}
\hline \multirow{8}{*}{$\frac{1}{2}(B+C)=$} & $\mathrm{HN}^{14} \mathrm{C}^{12} \mathrm{~S}^{32}$ & $\mathrm{DN}^{14} \mathrm{C}^{12} \mathrm{~S}^{32}$ & $\mathrm{HN}^{14} \mathrm{C}^{13} \mathrm{~S}^{32}$ & $\mathrm{DN}^{14} \mathrm{C}^{13} \mathrm{~S}^{32}$ & $\mathrm{HN}^{14} \mathrm{C}^{12} \mathrm{~S}^{34}$ \\
\hline & 5866.0 & 5474.3 & 5847.3 & 5459.8 & 5728.8 \\
\hline & & $J=1-2$ & Frequency & & \\
\hline & & $\mathrm{HN}^{14} \mathrm{C}^{12} \mathrm{~S}^{32}$ & 23,464 & & \\
\hline & & $\mathrm{DN}^{14} \mathrm{C}^{12} \mathrm{~S}^{32}$ & 21,897 & & \\
\hline & & $\mathrm{HN}^{14} \mathrm{C}^{13} \mathrm{~S}^{32}$ & 23,389 & [1] & \\
\hline & & $\mathrm{DN}^{14} \mathrm{C}^{13} \mathrm{~S}^{32}$ & 21,839 & & \\
\hline & & $\mathrm{HN}^{14} \mathrm{C}^{12} \mathrm{~S}^{34}$ & 22,915 & & \\
\hline
\end{tabular}
}

$\mathrm{H}_{2} \mathrm{O}$ (Water). The water line has been investigated at higher pressures by several workers not quoted herein. The intensity of the HDO lines follows the procedure adopted throughout this table of using natural abundancies where possible, the abundance of $\mathrm{D}$ being taken as 0.02 percent.

$$
\begin{array}{rlll}
\mathrm{H}_{2} \mathrm{O} & \text { Reference } & \text { HDO } & \\
\mu=1.94 & {[20]} & 1.84 & {[49 \mathrm{~b}, 48]} \\
\Delta \nu=7 & {[2,55]} & 6.5 & {[49 \mathrm{~b}]}
\end{array}
$$

$\begin{array}{llllr}\mathrm{H}_{2} \mathrm{O}^{16} & \text { Transition } & \text { Frequency } & \text { Intensity } & \\ & 5_{-1}-6_{-5} & 22,235.22 & 9.6 \times 10^{-6} & {[20,24,55]} \\ \mathrm{HDO}^{16} & 2_{-1}-2_{-2} & 10,278.99 & 8.6 \times 10^{-9} & {[49 \mathrm{~b}]} \\ & 4_{-3}-3_{-1} & 20,460.40 & \mathbf{1 . 3} 3 \times 10^{-8} & {[44,49 \mathrm{~b}]} \\ & 5_{0}-5_{1} & 22,307.67 & \mathbf{1 . 0} 0 \times 10^{-8} & {[24,48]} \\ & 3_{0}-3_{1} & 50,236.90 & \mathbf{1 . ~} 1 \times 10^{-7} & {[49 \mathrm{~b}}\end{array}$

\footnotetext{
HNCS (Isothiocyanic acid). 
ICl. The half widths, dipole moment and intensities are discussed in reference [54].

$$
\begin{aligned}
\mu & =0.65 \\
B_{e} & =3422.300 \\
(e q Q)_{\mathrm{C} 135} & =-82.5 \\
(e q Q)_{I} & =-2930.0 \\
& =16.060 \\
\Delta \nu_{3-4} & =5.5 \\
\Delta \nu_{0-1} & =3.15
\end{aligned}
$$

$$
\begin{aligned}
& \mathrm{ICl}^{35} \\
& J=0-1, F_{1}=5 / 2-7 / 2 \\
& J=3-4, v=0 \\
& F_{l i} \quad F_{i} \quad F_{l f} \quad F_{f} \\
& 3 / 2 \quad 2 \quad 5 / 2 \quad 3 \\
& \begin{array}{llllll}
3 / 2 & 3 & 5 / 2 & 4 & 27,204.99 & 1.5 \times 10^{-6}
\end{array} \\
& 5 / 2 \quad 3 \quad 7 / 2 \quad 4 \quad 27,217.51 \quad 1.4 \times 10^{-6} \\
& 5 / 2 \quad 2 \quad 7 / 2 \quad 3 \quad 27,221.02 \quad 1.0 \times 10^{-6} \\
& 5 / 2 \quad 4 \quad 7 / 2 \quad 5 \quad 27,225.32 \quad 2.0 \times 10^{-6} \text {. } \\
& \begin{array}{llllll}
5 / 2 & 1 & 7 / 2 & 2 & 27,228.34 & 7.3 \times 10^{-7}
\end{array} \\
& \begin{array}{llllll}
1 / 2 & 1 & 3 / 2 & 2 & 27,242.59 & 3.9 \times 10^{-7}
\end{array} \\
& \begin{array}{llllll}
1 / 2 & 2 & 3 / 2 & 3 & 27,254.90 & 1.1 \times 10^{-6}
\end{array} \\
& 7 / 2,9 / 2 \\
& 9 / 2 \\
& 7 / 2,9 / 2 \\
& 9 / 2 \\
& 7 / 2,9 / 2 \\
& \begin{array}{rrr}
7 / 2,9 / 2 & & 9 / 2 \\
7 / 2,9 / 2 & 5 & 11 / 2
\end{array} \\
& 11 / 2 \quad 5 \quad 13 / 2 \\
& 11 / 2 \quad 6 \quad 13 / 2 \\
& 11 / 2 \quad 4 \quad 13 / 2 \\
& 11 / 2 \quad 7 \quad 13 / 2 \\
& 9 / 2 \quad 3 \quad 11 / 2 \\
& 9 / 2 \quad 4 \quad 11 / 2 \\
& \begin{array}{lll}
9 / 2 & 6 & 11 / 2
\end{array} \\
& 9 / 2 \quad 5 \quad 11 / 2 \\
& 11 / 2 \quad 13 / 2
\end{aligned}
$$

\begin{abstract}
6,684
\end{abstract}
ICN (Cyanogen iodide).

$$
\begin{aligned}
\mu= & 3.71 \\
B \mathrm{O}= & 3225.527 \text { for } \mathrm{IC}^{12} \mathrm{~N} \\
& 3177.035 \text { for } \mathrm{IC}^{13} \mathrm{~N} \\
(e q Q)_{N}= & 3.80 \\
(e q Q)_{I} & =-2420 . \\
\Delta \nu & =20 . \\
\alpha_{1} & =9.33 \\
\alpha_{2}= & =-9.50 \\
q & =2.69
\end{aligned}
$$

$\mathrm{IC}^{12} \mathrm{~N}^{14}$

$$
J=3-4, v=0 \begin{array}{rrll}
F_{i} & \multicolumn{1}{c}{F_{f}} & \text { Frequency } & \text { Intensity } \\
11 / 2 & 11 / 2 & 25,393.87 & 7.1 \times 10^{-6} \\
3 / 2 & 5 / 2 & 25,711.50 & 2.0 \times 10^{-5} \\
5 / 2 & 7 / 2 & 25,728.77 & 2.9 \times 10^{-5} \\
1 / 2 & 3 / 2 & 25,752.65 & 1.4 \times 10^{-5} \\
7 / 2 & 9 / 2 & 25,783.50 & 4.2 \times 10^{-5} \\
9 / 2 & 9 / 2 & 25,789.85 & 1.1 \times 10^{-5} \\
11 / 2 & 13 / 2 & 25,823.08 & 7.5 \times 10^{-5} \\
9 / 2 & 11 / 2 & 25,837.64 & 5.7 \times 10^{-5} \\
7 / 2 & 7 / 2 & 25,954.36 & 1.2 \times 10^{-5} \\
3 / 2 & 3 / 2 & 25,969.58 & 6.9 \times 10^{-6} \\
5 / 2 & 5 / 2 & 25,991.92 & 1.0 \times 10^{-5}
\end{array}
$$

$$
\left.\begin{array}{rlll}
v_{1}=1 & & & \\
11 / 2 & 13 / 2 & 25,748.18 & 7.0 \times 10^{-6} \\
9 / 2 & 11 / 2 & 25,763.23 & 5.3 \times 10^{-6}
\end{array}\right\}
$$

$$
v_{2}=1
$$$$
1_{1} \quad 5 / 2 \quad 7 / 2 \quad 25,802.92
$$$$
1_{1} \quad 3 / 2 \quad 5 / 2 \quad 25,815.34
$$$$
1_{1} \quad 7 / 2 \quad 9 / 2 \quad 25,829.31
$$$$
1_{2} \quad 7 / 2 \quad 9 / 2 \quad 25,850.78
$$$$
1_{1} \quad 9 / 2 \quad 11 / 2 \quad 25,872.24
$$$$
1_{2} \quad 9 / 2 \quad 11 / 2 \quad 25,893.73
$$$$
1_{1} \quad 11 / 2 \quad 13 / 2 \quad 25,906.28
$$$$
1_{2} \quad 11 / 2 \quad 13 / 2 \quad 25,927.66
$$

3. $8 \times 10^{-6}$

3. $8 \times 10^{-6}$

7. $8 \times 10^{-6}$

7. $8 \times 10^{-6}$

1. $1 \times 10^{-5}$

1. $1 \times 10^{-5}$

1. $4 \times 10^{-5}$

1. $4 \times 10^{-5}$

$$
\begin{aligned}
& v_{2}=2 \\
& l=2
\end{aligned}
$$$$
11 / 2 \quad 13 / 2 \quad 1_{2} \quad 26,046.32
$$$$
\text { 3. } 5 \times 10^{-6} \mathrm{P}
$$

77,413 .

7. $7 \times 10^{-3}$

[41]

83, 864 .

9. $7 \times 10^{-3}$

[53] $\mathrm{IC}^{13} \mathrm{~N}^{14}$

[42] $J=4-5, v=0$

[53]

$\begin{array}{rr}5 / 2 & 7 / 2 \\ 7 / 2 & 9 / 2 \\ 3 / 2 & 5 / 2 \\ 9 / 2 & 11 / 2 \\ 13 / 2 & 15 / 2 \\ 11 / 2 & 13 / 2\end{array}$

$31,718.28$
$31,730.50$
$31,741.50$
$31,763.34$
$31,783.31$
$31,793.46$

$\left.\begin{array}{l}\text { 5. } 5 \times 10^{-7} \\ \text { 7. } 2 \times 10^{-7} \\ \text { 4. } 3 \times 10^{-7} \\ \text { 9. } 1 \times 10^{-7} \\ \text { 1. } 4 \times 10^{-6} \\ \text { 1. } 1 \times 10^{-6}\end{array}\right\}$


$\mathrm{NH}_{3}$ (Ammonia). The ammonia spectrum is unusual in being produced primarily by inversion or vibration rather than rotation of the molecule. It has considerably greater intensity than other micro-wave spectra of the same frequency, with the result that it is the first known and most widely studied microwave spectrum. The dependence of inversion frequency on the rotational quantum numbers $J$ and $K$ of the molecule produces a "fine" structure with frequencies given by the formula [45]

$$
\begin{aligned}
\nu= & 23,785.75-151.450\left(J^{2}+J-K^{2}\right)+59.892 K^{2}+ \\
& 0.49569\left(J^{2}+J-K^{2}\right)^{2}-0.37674 K^{2}\left(J^{2}+J-K^{2}\right)+ \\
& 0.06554 K^{4} .
\end{aligned}
$$

This formula applies with an accuracy of about 2 me for small $J$ and $K$ up to about $J=12$ except when $K=3$, for which deviations are somewhat larger [45, 34]. The expression [40]

$$
\begin{aligned}
& \nu=23,787-151.3 J(J+1)+211.0 K^{2}+0.5503 J^{2}(J+1)^{2}- \\
& 1.531 J(J+1)+1.055 K^{4},
\end{aligned}
$$

applies with an accuracy of about 30 me up to $J=16$. Each one of the lines listed for $\mathrm{N}^{14} \mathrm{H}_{3}$ may be expected to show hyperfine structure due to the $\mathrm{N}^{14}$ quadrupole coupling [7a, 58a]. Additional small frequency shifts of this hyperfine structure due to the $\mathrm{N}^{14}$ nuclear magnetic coupling have been detected $[40,24 a, 27 a]$. The resulting symmetric patterns of two weak lines on each side of the lines listed have been studied up to $J=9$ [40], but the weak lines are not listed in this table. The frequency difference between these lines and the strong component that is listed are given by

$$
\begin{aligned}
& \delta \nu= \\
& \pm\left\{\frac{3}{4} e q Q\left[1-\frac{3 K^{2}}{J(J+1)}\right] \frac{J+1}{2 J+3}-J\left[\frac{0.0104 K^{2}}{J(J+1)}+0.0022\right]\right\}, \\
& \delta \nu^{\prime}= \\
& \pm\left\{\frac{3}{4} e q Q\left[1-\frac{3 K^{2}}{J(J+1)}\right] \frac{J}{2 J-1}+(J+1)\left[\frac{0.0104 K^{2}}{J(J+1)}+0.0022\right]\right\} .
\end{aligned}
$$

The sign of eqQ cannot be definitely measured experimentally, but is very probably negative, and is assumed negative in the expressions for $\delta \nu$ and $\delta \nu^{\prime} . \quad \mathrm{N}^{15} \mathrm{H}_{3}$ shows no hyperfine structure, since the $\mathrm{N}^{15}$ quadrupole moment is zero. Intensities of the weak hyperfine components relative to the strong component decrease approximately as $1 / J^{2}$. The rather involved expressions for exact intensities are given by Candler [4c] and (with some typographical errors) in reference [40].

Intensities listed in the table are for the entire fine structure lines (without resolution of hyperfine components) assuming for each line, including the $\mathrm{N}^{15} \mathrm{H}_{3}$ lines, the same value of half width $\Delta \nu$ measured for the $\mathrm{N}^{14} \mathrm{H}_{3} J=3, K=3$ line[50]*. Bleaney et al.[4a] find actually that for a number of $\mathrm{NH}_{3}$ lines $\Delta \nu$ depends on $J$ and $K$

\begin{tabular}{|c|c|}
\hline $\mathrm{N}^{14} \mathrm{H}_{3}$ & $\mathrm{~N}^{15} \mathrm{H}_{3}$ \\
\hline$\mu=1.45$ & 1. 45 \\
\hline$\Delta \nu=28$ & \\
\hline$A_{0}=189,000$ & 189,00 \\
\hline$B_{0}=298,000$ & \\
\hline$e q Q=-4.10$ & \\
\hline
\end{tabular}
according to the formula

$$
\Delta \nu \alpha\left[\frac{K^{2}}{J(J+1)}\right]^{1 / 3}
$$

so that intensities should be obtained from those in the table by multiplying by the factor $\left[3 J(J+1) / 4 K^{2}\right]^{1 / 3}$. The apparent frequencies and values of $\Delta \nu$ at atmospheric and higher pressures differ widely from those given here [4a. $59 \mathrm{a}, 4 \mathrm{~b}]$. See also [33a].

$\mathrm{NH}_{3}$ (Ammonia).

[50]

0

[40] 
$\mathrm{N}^{14} \mathrm{H}_{3}$

$\begin{array}{rr}J & K \\ 7 & 4 \\ 6 & 3 \\ 5 & 1 \\ 5 & 2 \\ 8 & 6 \\ 9 & 7 \\ 7 & 5 \\ 10 & 8 \\ 6 & 4 \\ 11 & 9 \\ 4 & 1 \\ 5 & 3 \\ 12 & 10 \\ 4 & 2 \\ 3 & 1 \\ 14 & 12 \\ 5 & 4 \\ 4 & 3 \\ 6 & 5 \\ 3 & 2 \\ 7 & 6 \\ 15 & 13 \\ 2 & 1 \\ 8 & 7 \\ 9 & 8 \\ 1 & 1 \\ 13 \\ 2 & 2 \\ 3 & 3 \\ 4 & 4 \\ 10 & 9 \\ 5 & 5 \\ 11 & 10 \\ 12 & 13 \\ 13 & 10 \\ 12 & 12 \\ 13 & 13 \\ 16 & 14 \\ & 16\end{array}$

Frequency

19, 218. 52

$19,757.56$

19,829

$20,371.48$

20, 719. 20

20, 735. 46

$20,804.80$

$20,852.51$

20, 994. 62

21, 070. 73

$21,134.37$

$21,285.30$

21, 391. 55

21, 703. 34

$22,234.51$

22,355

$22,653.00$

$22,688,24$

$22,732,45$

$22,834.10$

22, 924.91

23, 004

$23,098.78$

$23,232.20$

$23,657.46$

$23,694.48$

$23,722.61$

$23,870.11$

$24,139.45$

24, 205. 25

24, 532. 94

$24,881.90$

25, 056. 04

25, 695. 23

$25,715.14$

26, 518. 91

$26,655.00$

27, 478. 00

$27,772.52$

28, 604. 73

29, 061. 14

29, 914. 66

31, 424. 97

33, 156. 95

$35,134.44$

37, 385. 18

$39,941.54$
Intensity

2. $9 \times 10^{-5}$

6. $8 \times 10^{-5}$

6. $9 \times 10^{-6}$

3. $1 \times 10^{-5}$

8. $8 \times 10^{-5}$

2. $9 \times 10^{-5}$

6. $2 \times 10^{-5}$

1. $7 \times 10^{-5}$

7. $8 \times 10^{-5}$

1. $9 \times 10^{-5}$

1. $6 \times 10^{-5}$

1. $7 \times 10^{-4}$

4. $9 \times 10^{-6}$

6. $9 \times 10^{-5}$

3. $3 \times 10^{-5}$

2. $1 \times 10^{-6}$

1. $9 \times 10^{-4}$

3. $7 \times 10^{-4}$

1. $6 \times 10^{-4}$

1. $5 \times 10^{-4}$

2. $7 \times 10^{-4}$

4. $7 \times 10^{-7}$

6. $7 \times 10^{-5}$

9. $5 \times 10^{-5}$

6. $3 \times 10^{-5}$

1. $6 \times 10^{-4}$

3. $0 \times 10^{-4}$

7. $9 \times 10^{-4}$

4. $3 \times 10^{-4}$

7. $8 \times 10^{-5}$

4. $1 \times 10^{-4}$

2. $2 \times 10^{-5}$

7. $1 \times 10^{-4}$

1. $3 \times 10^{-5}$

2. $8 \times 10^{-4}$

2. $1 \times 10^{-4}$

1. $3 \times 10^{-5}$

2. $9 \times 10^{-4}$

3. $0 \times 10^{-6}$

9. $5 \times 10^{-5}$

1. $4 \times 10^{-6} \quad[40]$

5. $8 \times 10^{-5} \quad[40]$

6. $6 \times 10^{-5} \quad[40]$

1. $8 \times 10^{-5} \quad[40]$

9. $2 \times 10^{-6} \quad$ [40]

8. $9 \times 10^{-6} \quad[40]$

2. $0 \times 10^{-6} \quad[40]$
Earlier references by

workers other

than those

already quoted

[4]

$5,21]$

$[45,21]$

[4]

[4]

[4]

[4]

[4]

[4]

[4]

[4]

[4]

[4]

[4]

[4]

[4]

[4]

$[4,50]$

$[4,50]$

$[4,50]$

$[4,50]$

$[4,50]$

$[4,50]$

$[4,50]$

$[4,50]$

$[4,50]$

$[4,50]$

$[4,50]$

[50]

$[4,50]$

[4] 
$\mathrm{N}^{15} \mathrm{H}_{3}$

$$
\left.\begin{array}{rrll}
J & K & \text { Frequency } & \text { Intensity } \\
5 & 3 & 20,272.04 & 6.4 \times 10^{-7} \\
4 & 2 & 20,682.87 & 2.6 \times 10^{-7} \\
3 & 1 & 21,202.30 & 1.3 \times 10^{-7} \\
5 & 4 & 21,597.86 & 7.2 \times 10^{-7} \\
4 & 3 & 21,637.91 & 1.4 \times 10^{-6} \\
6 & 5 & 21,667.93 & 6.0 \times 10^{-7} \\
3 & 2 & 21,783.98 & 5.6 \times 10^{-7} \\
7 & 6 & 21,846.41 & 9.9 \times 10^{-7} \\
2 & 1 & 22,044.28 & 2.6 \times 10^{-7} \\
8 & 7 & 22,134.89 & 3.5 \times 10^{-7} \\
9 & 8 & 22,536.26 & 2.3 \times 10^{-7} \\
1 & 1 & 22,624.96 & 6.0 \times 10^{-7} \\
2 & 2 & 22,649.85 & 1.2 \times 10^{-6} \\
3 & 3 & 22,789.41 & 3.0 \times 10^{-6} \\
4 & 4 & 23,046.10 & 1.6 \times 10^{-6} \\
10 & 9 & 23,054.97 & 3.0 \times 10^{-7} \\
5 & 5 & 23,421.99 & 1.5 \times 10^{-6} \\
6 & 6 & 23,922.32 & 2.6 \times 10^{-6} \\
7 & 7 & 24,553.42 & 1.1 \times 10^{-6} \\
8 & 8 & 25,323.51 & 8.0 \times 10^{-7}
\end{array}\right\}
$$

$\mathrm{N}_{2} \mathrm{O}$ (Nitrous oxide)

$$
\begin{aligned}
\Delta \nu & =3.35 \\
\mu & =.16
\end{aligned}
$$

\begin{tabular}{|c|c|c|c|c|}
\hline & $0^{16}$ & & $\mathrm{~N}^{15} \mathrm{~N}^{14} 0^{16}$ & \\
\hline$B_{0}=$ & 561.6 & & $12,137.31$ & [7] \\
\hline$(e q Q)_{N}=$ & $\begin{array}{r}\text { centro } \\
-.2\end{array}$ & & $\begin{array}{l}\text { end } \\
-1.03\end{array}$ & {$[7,43]$} \\
\hline & $F_{i}$ & $F_{f}$ & Frequency & Intensity \\
\hline$J=0-1$ & 1 & 1 & $25,123.03$ & 1. $1 \times 10^{-5}$ \\
\hline & 1 & 2 & $25,123.28$ & 1. $7 \times 10^{-5}$ \\
\hline & 1 & 0 & $25,123.64$ & 3. $4 \times 10^{-6}$ \\
\hline $\mathrm{N}^{15} \mathrm{~N}^{14} \mathrm{O}^{16}$ & & & & \\
\hline$J=0-1$ & 1 & 1 & $24,274.53$ & $3.5 \times 10^{-8}$ \\
\hline & 1 & 2 & $24,274.61$ & 5. $8 \times 10^{-8}$ \\
\hline & 1 & 0 & $24,274.73$ & 1. $1 \times 10^{-8}$ \\
\hline $\mathrm{N}^{14} \mathrm{~N}^{15} \mathrm{O}^{16}, J=$ & & & $25,121.55$ & 1. $1 \times 10^{-7}$ \\
\hline $\mathrm{N}^{15} \mathrm{~N}^{15} \mathrm{O}^{16}, J=$ & & & $24,274.78$ & 4. $0 \times 10^{-10}$ \\
\hline
\end{tabular}

$\mathrm{O}_{2}$ (Oxygen). This spectrum is of a fundamentally different nature than the other molecular spectra recorded herein, being due to a magnetic coupling to the electromagnetic field rather than an electric dipole coupling. The theory of the transition has been discussed by Van Vleck [58b].

$\begin{aligned} \mathrm{O}_{2}^{16} & \Delta \nu=.8-1.3 \\ & \text { Frequency } \\ & \text { Ca. 60,000 [3, 31, 48a }]\end{aligned}$

OCS (Carbonyl sulfide).

$$
\begin{aligned}
\mu\left(\mathrm{O}^{16} \mathrm{C}^{12} \mathrm{~S}^{32}\right) & =.732 & & {[16,27] } \\
\mu_{\mathrm{v} 2}=1 & =.694 & & {[49 \mathrm{c}] } \\
\mu\left(\mathrm{O}^{16} \mathrm{C}^{13} \mathrm{~S}^{32}\right) & =.722 & & {[16,27] } \\
(e q Q) \mathrm{S}^{33} & =-28.5 & & {[52] } \\
\Delta \nu & =6 . & & {[53] }
\end{aligned}
$$

$\begin{array}{lrrrrrrr} & \mathrm{O}^{16} \mathrm{C}^{12} \mathrm{~S}^{32} & \mathrm{O}^{16} \mathrm{C}^{12} \mathrm{~S}^{33} & \mathrm{O}^{16} \mathrm{C}^{12} \mathrm{~S}^{34} & \mathrm{O}^{16} \mathrm{C}^{13} \mathrm{~S}^{32} & \mathrm{O}^{16} \mathrm{C}^{13} \mathrm{~S}^{34} & \mathrm{O}^{16} \mathrm{C}^{14} \mathrm{~S}^{32} & \mathrm{O}^{18} \mathrm{C}^{12} \mathrm{~S}^{32} \\ \mathrm{~B}_{0} & 6081.453 & 6004.918 & 5932.843 & 6061.939 & 5911.730 & 6043.25 & 5704.825 \\ \alpha_{1} & 18.12 & & & & & -9.4 \\ \alpha_{2} & -10.59 & & -10.37 & & & 6.7 \\ \alpha_{3} & 55.1 & & 6.07 & & & 6.7 \\ q & 6.393 & & 6.07 & & & & \\ & {[16]} & {[52]} & {[53]} & {[53]} & {[53]} & {[37]}\end{array}$

The $\mathrm{B}_{0}$ 's listed above are those for the lowest observed $J$ transition, since they may be expected to increase with $J$ because of centrifugal stretching. $\mathrm{O}^{16} \mathrm{C}^{12} \mathrm{~S}^{32}$

$$
\begin{aligned}
& J=1-2, v=0 \\
& v_{1}=1 \\
& v_{2}=1,1_{1} \\
& v_{2}=2^{1_{2}} \\
& \left.\nu_{2}=3\right\} \\
& J=2-3, v=0 \\
& J=3-4, v=0 \\
& J=4-5, v=0 \\
& \mathrm{O}^{16} \mathrm{C}^{12} \mathrm{~S}^{33}
\end{aligned}
$$$$
\text { Frequency }
$$

\section{Intensity}

5. $5 \times 10^{-5}$

8. $7 \times 10^{-7}$

4. $4 \times 10^{-6}$

4. $4 \times 10^{-6}$

1. $3 \times 10^{-6}$

$1 \times 10^{-7}$

$1 \times 10^{-7}$

4. $6 \times 10^{-5}$

4. $4 \times 10^{-4}$

8. $7 \times 10^{-4}$$$
\text { 24, 325. } 92
$$$$
\text { 24, 253. } 44
$$$$
24,355.50
$$

[16]

[52]

[16]

[39b]

[39b]

[39b]

36, 488. 82

48, 651. 64

$60,814.08$
[49]

[27]

[27]
$F_{i} \quad F f$

$J=1-2, \quad 1 / 2 \quad 3 / 2$

$v=0 \quad 5 / 2 \quad 5 / 2$

$\begin{array}{ll}1 / 2 & 1 / 2\end{array}$

$3 / 2 \quad 5 / 2$

$5 / 2 \quad 7 / 2$

$3 / 2 \quad 3 / 2$

$3 / 2 \quad 1 / 2$

$\mathrm{O}^{16} \mathrm{C}^{12} \mathrm{~S}^{34}$

24, 013.04

7. $2 \times 10^{-8}$

[52]

24, 020. 21

2. $9 \times 10^{-7}$

24, 025. 39

4. $6 \times 10^{-8}$

7. $2 \times 10^{-9}$

$\mathrm{J}=1-2, v=0$

$v_{1}=1$

$v_{2}=1,1_{1}$

$\mathrm{J}=3-4, v=0$

$\mathrm{O}^{16} \mathrm{C}^{12} \mathrm{~S}^{36}$

$J=1-2, v=0$
2. $2 \times 10^{-6}$

4. $1 \times 10^{-8}$

1. $4 \times 10^{-7}$

1. $4 \times 10^{-7}$

1. $9 \times 10^{-5}$

$8 \times 10^{-9}$ 
$\mathrm{O}^{16} \mathrm{C}^{13} \mathrm{~S}^{32}$

$\begin{array}{clll}J=1-2, v=0 & \text { Frequency } & \text { Intensity } & \\ v_{2}=1,1_{1} & 24,247.76 & 5.9 \times 10^{-7} & {[53]} \\ 1_{2} & 24,275.25 & 6.6 \times 10^{-8} & {[53]} \\ \mathrm{O}^{16} \mathrm{C}^{13} \mathrm{~S}^{34} & & 24,301.05 & 6.6 \times 10^{-8}\end{array}$

$\mathrm{O}^{16} \mathrm{C}^{14} \mathrm{~S}^{32}$

$J=1-2, v=0$

$23,646.92 \quad 2.8 \times 10^{-8}$

$\begin{array}{ll}24,173.0 & 5.7 \times 10^{-5} \\ 24,197 & \left(100 \% \mathrm{C}^{14}\right) \\ 24,224 & 6.4 \times 10^{-6} \\ 23,534.67 & 2.2 \times 10^{-6} \\ 22,819.30 & 9.6 \times 10^{-8}\end{array}$

$\mathrm{O}^{18} \mathrm{C}^{12} \mathrm{~S}^{32}$

$J=1-2, v=0$

OCSe (Carbonyl selenide).

$$
\begin{array}{r}
\mu=.754 \\
\mu_{v=1}=.728 \\
\mu_{v=1}=.730
\end{array}
$$

\begin{tabular}{rl} 
Molecule & \multicolumn{1}{c}{$B_{000}$} \\
${ }^{16} \mathrm{C}^{12} \mathrm{Se}^{82}$ & $3994.009 \pm .005$ \\
$\mathrm{Se}^{80}$ & 4017.677 \\
$\mathrm{Se}^{78}$ & 4042.460 \\
$\mathrm{Se}^{77}$ & 4055.300 \\
$\mathrm{Se}^{76}$ & 4068.465 \\
$\mathrm{Se}^{74}$ & 4095.793 \\
${ }^{16} \mathrm{C}^{13} \mathrm{Se}^{80}$ & 3980.045 \\
$\mathrm{Se}^{78}$ & 4005.112
\end{tabular}

$\mathrm{O}^{16} \mathrm{C}^{12} \mathrm{Se}^{74}$

$$
\begin{aligned}
J=2-3, v & =0 \\
\mathrm{O}^{16} \mathrm{C}^{12} \mathrm{Se}^{76} & \\
J=2-3, v & =0 \\
v_{2} & =1,1_{1} \\
v_{2} & =1,1_{2} \\
\mathrm{O}^{16} \mathrm{C}^{12} \mathrm{Se}^{77} & \\
J=2-3, v & =0 \\
v_{1} & =1 \\
v_{2} & =1,1_{1} \\
v_{2} & =1,1_{2}
\end{aligned}
$$$$
\mathrm{O}^{16} \mathrm{C}^{12} \mathrm{Se}^{78}
$$$$
J=2-3, v=0
$$$$
\begin{aligned}
& v_{1}=1 \\
& v_{2}=1,1_{1} \\
& v_{2}=1,1_{2}
\end{aligned}
$$$$
J=5-6
$$$$
J=6-7
$$$$
\mathrm{O}^{16} \mathrm{C}^{12} \mathrm{Se}^{80}
$$$$
\begin{aligned}
J=2-3, v & =0 \\
v_{1} & =1 \\
v_{2} & =1,1_{1} \\
v_{2} & =1,1_{2}
\end{aligned}
$$$$
J=5-6
$$$$
J=6-7
$$$$
\mathrm{O}^{16} \mathrm{C}^{12} \mathrm{Se}^{82}
$$$$
J=2-3, v=0
$$$$
v_{1}=1
$$$$
v_{2}=1,1_{1}
$$$$
J=6-7
$$$$
v_{2}=1,1_{2}
$$

$\left.\begin{array}{ll}\text { Frequency } & \text { Intensity } \\ 24,574.67 & 1.3 \times 10^{-7} \\ & \\ 24,410.70 & 1.4 \times 10^{-6} \\ 24,442.98 & 1.8 \times 10^{-7} \\ 24,462.42 & 1.8 \times 10^{-7} \\ & \\ 24,331.71 & 1.2 \times 10^{-6} \\ 24,250.84 & 5.5 \times 10^{-8} \\ 24,363.97 & 1.6 \times 10^{-7} \\ 24,383.21 & 1.6 \times 10^{-7} \\ & \\ 24,254.67 & 3.4 \times 10^{-6} \\ 24,174.30 & 1.6 \times 10^{-8} \\ 24,286.82 & 4.5 \times 10^{-7} \\ 24,305.95 & 4.5 \times 10^{-7} \\ 48,508.88 & 2.8 \times 10^{-5} \\ 56,593.16 & 4.4 \times 10^{-5} \\ & \\ 24,105.98 & 6.8 \times 10^{-6} \\ 24,026.39 & 3.1 \times 10^{-7} \\ 24,138.05 & 9.0 \times 10^{-7} \\ 24,156.93 & 9.0 \times 10^{-7} \\ 48,211.46 & 5.6 \times 10^{-5} \\ 56,246.47 & 8.8 \times 10^{-5} \\ & \\ 23,964.50 & 1.3 \times 10^{-6} \\ 23,885.76 & 6.0 \times 10^{-8} \\ 23,996.26 & 1.7 \times 10^{-7} \\ 24,014.97 & 1.7 \times 10^{-7} \\ 55,916.19 & 1.1 \times 10^{-5} \\ & \\ 24,030.58 & 3.7 \times 10^{-8} \\ 23,880.18 & 7.5 \times 10^{-8}\end{array}\right\}$

$\mathrm{O}^{16} \mathrm{C}^{13} \mathrm{Se}^{78}$

$J=2-3, v=0$

$\mathrm{O}^{16} \mathrm{C}^{13} \mathrm{Se}^{80}$

$23,880.18 \quad 7.5 \times 10^{-8}$

$\left.\begin{array}{llc}\begin{array}{c}\alpha_{2} \\ -6.86 \pm .005\end{array} & \multicolumn{1}{c}{q .12 \pm .005} & D_{v} \mathrm{c} / \mathrm{sec} \\ -6.92 & 3.15 & 875 \pm 50 \\ -6.96 & 3.19 & 855 \pm 20 \\ -6.98 & 3.21 & 830 \pm 50 \\ -7.00 & 3.24 & \end{array}\right\}$

$\mathrm{PF}_{3}$ (Phosphorus trifluoride)

$$
\begin{array}{ll}
\mu=1.02 & {[39 \mathrm{c}]} \\
\Delta \nu=16 . & {[19]} \\
B_{0}=7819.90 & {[19]}
\end{array}
$$

$$
\left.\begin{array}{lrl}
\mathrm{PF}_{3} & \text { Frequency } & \text { Intensity* } \\
J=1-2, K=0,1 & 31,279.60 & 2.3 \times 10^{-6} \\
J=2-3, K=0,1 & 46,918.82 & 6.7 \times 10^{-6} \\
K=2 & 46,919.02 & 1.5 \times 10^{-6}
\end{array}\right\}
$$

*Assuming $80 \%$ in ground vibrational state.

excited vibrational states $\quad\left\{\begin{array}{l}46,940 \\ 47,010 \\ 47,033 \\ 47,040\end{array}\right\}[19]$

$$
\begin{array}{rlr}
\mathrm{SiH}_{3} \mathrm{Cl} \text { (Chlorosilane) } & & \mu=1.28 \\
A_{0}=95,500 & & \mathrm{SiH}_{3} \mathrm{Cl}^{37} \\
B_{0}=\mathrm{SiH}_{3} \mathrm{Cl}^{35} & 6673.81 & 6,512.40 \\
(e q Q) \mathrm{Cl}=-40.0 & -30.8
\end{array}
$$

$$
\begin{aligned}
& \mathrm{SiH}_{3} \mathrm{Cl}^{35} \quad F_{i} \quad F_{f} \quad \text { Frequency Intensity* } \\
& J=1-2, K=0
\end{aligned}
$$

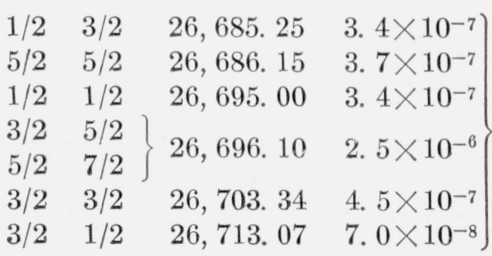




$\left.\begin{array}{rcccc}J=1-2, K=1 & F_{i} & F_{f} & \text { Frequency } & \text { Intensity } \\ & 3 / 2 & 5 / 2 & 26,687.34 & 6.6 \times 10^{-7} \\ 3 / 2 & 3 / 2 & 26,690.92 & 3.4 \times 10^{-7} \\ 5 / 2 & 5 / 2 & 26,692.66 & 2.8 \times 10^{-7} \\ 5 / 2 & 7 / 2 & 26,697.45 & 1.3 \times 10^{-6} \\ 1 / 2 & 3 / 2 & 26,700.48 & 2.6 \times 10^{-7} \\ 1 / 2 & 1 / 2 & 26,705.02 & 2.6 \times 10^{-7}\end{array}\right\}[38]$

$\mathrm{SiH}_{3} \mathrm{Cl}^{37}$

$J=1-2, K=0$

\begin{tabular}{|c|c|c|c|c|c|}
\hline & $1 / 2$ & $3 / 2$ & \multirow{2}{*}{$26,042.41$} & \multirow{2}{*}{\multicolumn{2}{|c|}{$2.2 \times 10^{-7} \quad[38]$}} \\
\hline & $5 / 2$ & $5 / 2$ & & & \\
\hline & $3 / 2$ & $5 / 2$ & \multirow{2}{*}{$26,050.26$} & \multirow{2}{*}{ 7. $9 \times 10^{-7}$} & \multirow{10}{*}[38]{} \\
\hline & $5 / 2$ & $7 / 2$ & & & \\
\hline & $3 / 2$ & $3 / 2$ & $26,055.86$ & 1. $4 \times 10^{-7}$ & \\
\hline & $3 / 2$ & $1 / 2$ & $26,063.52$ & 2. $2 \times 10^{-8}$ & \\
\hline \multirow[t]{6}{*}{$K=1$} & $3 / 2$ & $5 / 2$ & $26,043.29$ & 2. $0 \times 10^{-7}$ & \\
\hline & $3 / 2$ & $3 / 2$ & $26,046.30$ & 1. $0 \times 10^{-7}$ & \\
\hline & $5 / 2$ & $5 / 2$ & $26,047.97$ & 8. $7 \times 10^{-8}$ & \\
\hline & $5 / 2$ & $7 / 2$ & $26,051.09$ & 3. $9 \times 10^{-7}$ & \\
\hline & $1 / 2$ & $3 / 2$ & $26,053.35$ & 8. $1 \times 10^{-8}$ & \\
\hline & $1 / 2$ & $1 / 2$ & $26,057.10$ & 8. $1 \times 10^{-8}$ & \\
\hline
\end{tabular}

$\mathrm{SO}_{2}$ (Sulfur dioxide)

$$
\left.\begin{array}{rl}
\mu & =1.7 \\
A & =61,100 \\
B & =10,400 \\
C & =8,870
\end{array}\right\}[12]
$$

$\mathrm{S}^{32} \mathrm{O}_{2}^{16}$

$\left.\begin{array}{cc}\text { Transition } & \text { Frequency } \\ 13_{-10-12_{-6}} & 20,420 \\ & 22,064 \\ & 22,225 \\ & 22,475 \\ & 22,735 \\ & 22,904 \\ & 22,934 \\ & 23,033 \\ & 23,413 \\ 6-4-5_{-2} & 23,738 \\ & 24,037 \\ 9_{-8}-8_{-4} & 24,083 \\ & 24,342 \\ & 25,047 \\ & 25,170 \\ 7_{-4}-8_{-6} & 25,392 \\ 3_{-2}-4_{-4} & 29,460\end{array}\right\}[12]$

$\mathrm{SPF}_{3}$

$\mathrm{SPF}_{3}$

\begin{tabular}{|c|c|c|c|}
\hline Frequency & $\begin{array}{l}\text { Inten- } \\
\text { sity }\end{array}$ & Transition & Molecule \\
\hline 6,684 & W & $0-1$ & $\mathrm{ICl}^{37}$ \\
\hline 6,980 & M & $0-1$ & $\mathrm{ICl}^{35}$ \\
\hline $10,278.99$ & W & --- & $\mathrm{HDO}^{16}$ \\
\hline $17,961.2$ & M & $0-1$ & $\mathrm{~B}^{10} \mathrm{H}_{3} \mathrm{C}^{12} \mathrm{O}^{16}$ \\
\hline $18,398.6$ & M & $0-1$ & $\mathrm{C}^{12} \mathrm{H}_{3} \mathrm{C}^{12} \mathrm{~N}^{i 4}$ \\
\hline 18,400 & M & $0-1$ & $\mathrm{C}^{12} \mathrm{H}_{3} \mathrm{C}^{12} \mathrm{~N}^{14}$ \\
\hline $18,402.1$ & M & $0-1$ & $\mathrm{C}^{12} \mathrm{H}_{3} \mathrm{C}^{12} \mathrm{~N}^{14}$ \\
\hline $18,943.77$ & W & $0-1$ & $\mathrm{C}^{12} \mathrm{H}_{3} \mathrm{Br}^{81}$ \\
\hline $18,992.36$ & W & $0-1$ & $\mathrm{C}^{12} \mathrm{H}_{3} \mathrm{Br}^{79}$ \\
\hline $19,040.32$ & M & $0-1$ & $\mathrm{C}^{12} \mathrm{H}_{3} \mathrm{Br}^{8 !}$ \\
\hline $19,107.97$ & M & $0-1$ & $\mathrm{C}^{12} \mathrm{H}_{3} \mathrm{Br}^{79}$ \\
\hline $19,160.82$ & M & $0-1$ & $\mathrm{C}^{12} \mathrm{H}_{3} \mathrm{Br}^{81}$ \\
\hline $19,218.52$ & $\mathrm{~S}$ & $0-1$ & $\mathrm{~N}^{14} \mathrm{H}_{3}$ \\
\hline $19,252.13$ & M & $0-1$ & $\mathrm{C}^{12} \mathrm{H}_{3} \mathrm{Br}^{79}$ \\
\hline 19,700 & $\ldots \ldots$ & $3-4$ & $\mathrm{C}^{12} \mathrm{H}_{3} \mathrm{~N}^{14} \mathrm{C}^{12} \mathrm{~S}^{34}$ \\
\hline $19,757.56$ & $\mathrm{~S}$ & $-\cdots$ & $\mathrm{N}^{14} \mathrm{H}_{3}$ \\
\hline 19,829 & M & & $\mathrm{N}^{14} \mathrm{H}_{3}$ \\
\hline 19,929 & - & $3-4$ & $\mathrm{C}^{12} \mathrm{H}_{3} \mathrm{~N}^{14} \mathrm{C}^{12} \mathrm{~S}^{34}$ \\
\hline 20,020 & & $3-4$ & $\mathrm{C}^{12} \mathrm{H}_{3} \mathrm{~N}^{14} \mathrm{C}^{12} \mathrm{~S}^{32}$ \\
\hline 20,140 & $\ldots$ & $3-4$ & $\mathrm{C}^{12} \mathrm{H}_{3} \mathrm{~N}^{14} \mathrm{C}^{12} \mathrm{~S}^{32}$ \\
\hline 20,160 & & $3-4$ & $\mathrm{C}^{12} \mathrm{H}_{3} \mathrm{~N}^{14} \mathrm{C}^{12}$ \\
\hline 20,216 & - & $3-4$ & $\mathrm{C}^{12} \mathrm{H}_{3} \mathrm{~N}^{14} \mathrm{C}^{12} \mathrm{~S}^{32}$ \\
\hline 20,230 & & $3-4$ & $\mathrm{C}^{12} \mathrm{H}_{3} \mathrm{~N}^{14} \mathrm{C}^{12} \mathrm{~S}^{32}$ \\
\hline 20,241 & & $3-4$ & $\mathrm{C}^{12} \mathrm{H}_{3} \mathrm{~N}^{14} \mathrm{C}^{12} \mathrm{~S}^{32}$ \\
\hline $20,272.04$ & M & $-\ldots$ & $\mathrm{N}^{15} \mathrm{H}_{3}$ \\
\hline 20,350 & & $3-4$ & $\mathrm{C}^{12} \mathrm{H}_{3} \mathrm{~N}^{14} \mathrm{C}^{12} \mathrm{~S}^{32}$ \\
\hline $20,371.48$ & $\mathrm{~S}$ & & $\mathrm{~N}^{14} \mathrm{H}_{3}$ \\
\hline 20,385 & 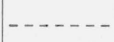 & & $\mathrm{CH}_{3} \mathrm{NO}_{2}$ \\
\hline 20,413 & & $3-4$ & $\mathrm{C}^{12} \mathrm{H}_{3} \mathrm{~N}^{14} \mathrm{C}^{12} \mathrm{~S}^{32}$ \\
\hline 20,443 & & $3-4$ & $\mathrm{C}^{12} \mathrm{H}_{3} \mathrm{~N}^{14} \mathrm{C}^{12} \mathrm{~S}^{32}$ \\
\hline 20,421 & & & $\mathrm{SO}_{2}$ \\
\hline $20,460.40$ & W & $-\ldots$ & $\mathrm{HDO}^{16}$ \\
\hline $20,679.9$ & W & $1-2$ & $\mathrm{CF}_{3} \mathrm{CH}_{3}$ \\
\hline $20,682.87$ & W & $-\ldots$ & $\mathrm{N}^{15} \mathrm{H}_{3}$ \\
\hline $20,710.6$ & M & $1-2$ & $\mathrm{CF}_{3} \mathrm{CH}_{3}$ \\
\hline $20,719.20$ & $\mathrm{~S}$ & -- & $\mathrm{N}^{14} \mathrm{H}_{3}$ \\
\hline $20,735.46$ & $\mathrm{~S}$ & $\ldots \ldots$ & $\mathrm{N}^{14} \mathrm{H}_{3}$ \\
\hline $20,742.3$ & M & $1-2$ & $\mathrm{CF}_{3} \mathrm{CH}_{3}$ \\
\hline $20,804.80$ & $\mathrm{~S}$ & & $\mathrm{~N}^{14} \mathrm{H}_{3}$ \\
\hline $20,852.51$ & $\mathrm{~S}$ & $\ldots$ & $\mathrm{N}^{14} \mathrm{H}_{3}$ \\
\hline 20,910 & - & - $\ldots \ldots$ & $\mathrm{CH}_{3} \mathrm{OH}$ \\
\hline 20,971 & & & $\mathrm{CH}_{3} \mathrm{OH}$ \\
\hline $20,994.62$ & $\mathrm{~S}$ & -- & $\mathrm{N}^{14} \mathrm{H}_{3}$ \\
\hline $21,070.73$ & $\mathrm{~S}$ & $-1--1-1$ & $\mathrm{~N}^{14} \mathrm{H}_{3}$ \\
\hline $21,134.37$ & $\mathrm{~S}$ & $--\infty-\infty-\infty$ & $\mathrm{N}^{14} \mathrm{H}_{3}$ \\
\hline $21,202.30$ & W & $\ldots$ & $\mathrm{N}^{15} \mathrm{H}_{3}$ \\
\hline 21,262 & & & $\mathrm{SPF}_{3}$ \\
\hline 21,283 & $\mid \ldots-\ldots$ & 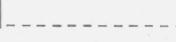 & $\mathrm{SPF}_{3}$ \\
\hline
\end{tabular}

$$
B_{0}=2657.6
$$

$$
\begin{gathered}
J=3-4 \\
J=4-5 \\
\text { Exc. states }
\end{gathered} \quad\left\{\begin{array}{l}
21,262 \\
26,574 \\
21,283 \\
26,553
\end{array}\right\}
$$

TABLE 2. Lines listed by frequency 
Table 2. Lines listed by frequency-Continued

\begin{tabular}{|c|c|c|c|}
\hline Frequency & $\begin{array}{l}\text { Inten- } \\
\text { sity }\end{array}$ & Transition & Molecule \\
\hline $21,285.30$ & S & & $\mathrm{N}^{14} \mathrm{H}_{3}$ \\
\hline 21,350 & & - . - . & $\mathrm{CH}_{3} \mathrm{OH}$ \\
\hline $21,391.55$ & M & $\ldots$ & $\mathrm{N}^{14} \mathrm{H}_{3}$ \\
\hline 21,482 & M & - - - - - - - & $\mathrm{CH}_{2} \mathrm{CF}_{2}$ \\
\hline 21,549 & W & 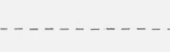 & $\mathrm{CH}_{2} \mathrm{CF}_{2}$ \\
\hline 21,551 & & - & $\mathrm{CH}_{3} \mathrm{OH}$ \\
\hline 21,573 & W & 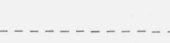 & $\mathrm{CH}_{2} \mathrm{CF}_{2}$ \\
\hline $21,597.86$ & M & - & $\mathrm{N}^{15} \mathrm{H}_{3}$ \\
\hline $21,637.91$ & M & 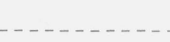 & $\mathrm{N}^{15} \mathrm{H}_{3}$ \\
\hline 21,664 & & 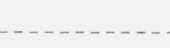 & $\mathrm{C}^{12} \mathrm{D}_{4} \mathrm{O}^{16}$ \\
\hline $21,667.93$ & M & $\ldots \ldots$ & $\mathrm{N}^{15} \mathrm{H}_{3}$ \\
\hline 21,689 & M & $-\ldots-n-\ldots-1$ & $\mathrm{CH}_{2} \mathrm{CF}_{2}$ \\
\hline $21,703.34$ & $\mathrm{~S}$ & - - & $\mathrm{N}^{14} \mathrm{H}_{3}$ \\
\hline 21,712 & & $\ldots$. & $\mathrm{CH}_{3} \mathrm{NH}_{2}$ \\
\hline 21,734 & M & 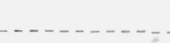 & $\mathrm{CH}_{2} \mathrm{CF}_{2}$ \\
\hline $21,783.98$ & M & - & $\mathrm{N}^{15} \mathrm{H}_{3}$ \\
\hline 21,839 & $\cdots$ & $1-2$ & $\mathrm{DN}^{14} \mathrm{C}^{13} \mathrm{~S}^{32}$ \\
\hline $21,846.41$ & M & $1-2$ & $\mathrm{DN}^{14} \mathrm{C}^{13} \mathrm{~S}^{32}$ \\
\hline 21,897 & 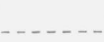 & $1-2$ & $\mathrm{DN}^{14} \mathrm{C}^{13} \mathrm{~S}^{32}$ \\
\hline $21,930.2$ & - & $1-2$ & $\mathrm{C}_{2} \mathrm{H}_{3} \mathrm{Cl}$ \\
\hline 21,935 & & - & $\mathrm{CH}_{3} \mathrm{NH}_{2}$ \\
\hline $22,044.28$ & W & $----1-1-1$ & $\mathrm{~N}^{15} \mathrm{H}_{3}$ \\
\hline 22,064 & & & $\mathrm{SO}_{2}$ \\
\hline $22,134.89$ & W & - - - n & $\mathrm{N}^{15} \mathrm{H}_{3}$ \\
\hline 22,217 & & 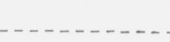 & $\mathrm{CHClF}_{2}$ \\
\hline 22,225 & & - - - - - - - & $\mathrm{SO}_{2}$ \\
\hline $22,234.51$ & $\mathrm{~S}$ & $-1--1-1-1$ & $\mathrm{~N}^{14} \mathrm{H}_{3}$ \\
\hline $22,235.22$ & M & - - - & $\mathrm{H}_{2} \mathrm{O}$ \\
\hline 22,236 & M & - - & $\mathrm{CH}_{2} \mathrm{CF}_{2}$ \\
\hline 22,247 & -- & - - - - & $\mathrm{CHClF}_{2}$ \\
\hline 22,258 & $\ldots$ & - - - - - & $\mathrm{CH}_{3} \mathrm{NH}_{2}$ \\
\hline 22,281 & $\mathrm{~S}$ & $-1-2-1-1-1$ & $\mathrm{CH}_{2} \mathrm{CF}_{2}$ \\
\hline 22,305 & & - n- & $\mathrm{CHClF}_{2}, \mathrm{CH}_{3} \mathrm{OH}$ \\
\hline $22,307.67$ & W & & HDO \\
\hline 22,353 & & $\ldots$ & $\mathrm{CHClF}_{2}$ \\
\hline 22,355 & M & $\ldots$ & $\mathrm{N}^{14} \mathrm{H}_{3}$ \\
\hline $22,369.6$ & $\cdots$ & $1-2$ & $\mathrm{C}_{2} \mathrm{H}_{3} \mathrm{Cl}$ \\
\hline 22,383 & M & $\ldots \ldots$ & $\mathrm{CH}_{2} \mathrm{CF}_{2}$ \\
\hline 22,386 & & . & $\mathrm{CHClF}_{2}$ \\
\hline 22,391 & M & - - - - & $\mathrm{CH}_{2} \mathrm{CF}_{2}$ \\
\hline 22,410 & & 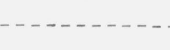 & $\mathrm{CHClF}_{2}$ \\
\hline 22,436 & & $----1---$ & $\mathrm{CHClF}_{2}$ \\
\hline 22,462 & & - - - - - - & $\mathrm{CHClF}_{2}$ \\
\hline 22,475 & & & $\mathrm{SO}_{2}$ \\
\hline 22,481 & & - - - & $\mathrm{CHClF}_{2}$ \\
\hline $22,485.9$ & & $1-2$ & $\mathrm{C}_{2} \mathrm{H}_{3} \mathrm{Cl}$ \\
\hline
\end{tabular}

TABLE 2. Lines listed by frequency-Continued

\begin{tabular}{|c|c|c|c|}
\hline Frequency & $\begin{array}{l}\text { Inten- } \\
\text { sity }\end{array}$ & Transition & Molecule \\
\hline 22,535 & & $-\ldots$ & $\mathrm{CH}_{3} \mathrm{NH}_{2}$ \\
\hline $22,536.26$ & W & $--1-1-1-1$ & $\mathrm{~N}^{15} \mathrm{H}_{3}$ \\
\hline 22,545 & & - - - - - - - & $\mathrm{CHClF}_{2}$ \\
\hline 22,553 & & - - - - - - - & $\mathrm{CHClF}_{2}$ \\
\hline 22,590 & & - . - . - & $\mathrm{CH}_{3} \mathrm{NH}_{2}$ \\
\hline 22,595 & & $\ldots$ & $\mathrm{CH}_{3} \mathrm{NH}_{2}$ \\
\hline 22,612 & & - - - - & $\mathrm{CH}_{3} \mathrm{NH}_{2}$ \\
\hline $22,624.96$ & M & $\ldots \ldots$ & $\mathrm{N}^{15} \mathrm{H}_{2}$ \\
\hline $22,649.85$ & M & - - - - & $\mathrm{N}^{15} \mathrm{H}_{3}$ \\
\hline $22,653.00$ & $\mathrm{~S}$ & $\ldots$ & $\mathrm{N}^{14} \mathrm{H}_{3}$ \\
\hline 22,660 & W & . n & $\mathrm{CH}_{2} \mathrm{CF}_{2}$ \\
\hline 22,680 & & $3-4$ & $\mathrm{C}^{12} \mathrm{H}_{3} \mathrm{~S}^{32} \mathrm{C}^{12} \mathrm{~N}^{14}$ \\
\hline $22,688.24$ & $\mathrm{~S}$ & $\ldots$ & $\mathrm{N}^{14} \mathrm{H}_{3}$ \\
\hline 22,732 & $\ldots$ & - & $\mathrm{CH}_{3} \mathrm{NH}_{2}$ \\
\hline $22,732.45$ & $\mathrm{~S}$ & - - - & $\mathrm{N}^{14} \mathrm{H}_{3}$ \\
\hline 22,735 & & ..... & $\mathrm{SO}_{2}$ \\
\hline 22,740 & ${ }^{2}$ & - - - - & $\mathrm{CH}_{3} \mathrm{NH}_{2}$ \\
\hline 22,752 & $\mathrm{~S}$ & $--1-1-1-1$ & $\mathrm{CH}_{2} \mathrm{CF}_{2}$ \\
\hline $22,789.41$ & M & $\ldots-\ldots-\ldots \ldots$ & $\mathrm{N}^{15} \mathrm{H}_{3}$ \\
\hline $22,819.30$ & W & $1-2$ & $\mathrm{O}^{18} \mathrm{C}^{12} \mathrm{~S}^{32}$ \\
\hline $22,834.10$ & $\mathrm{~S}$ & - & $\mathrm{N}^{14} \mathrm{H}_{3}$ \\
\hline 22,904 & & $\ldots$ & $\mathrm{SO}_{2}$ \\
\hline 22,915 & & $1-2$ & $\mathrm{HN}^{14} \mathrm{C}^{12} \mathrm{~S}^{34}$ \\
\hline $22,924.91$ & $\mathrm{~S}$ & - - - & $\mathrm{N}^{14} \mathrm{H}_{3}$ \\
\hline 22,934 & -- & $---\ldots-\ldots-n$ & $\mathrm{SO}_{2}$ \\
\hline $22,946.9$ & & $1-2$ & $\mathrm{C}_{2} \mathrm{H}_{3} \mathrm{Cl}$ \\
\hline 22,977 & & $-\ldots$ & $\mathrm{CH}_{3} \mathrm{NH}_{2}$ \\
\hline 23,004 & W & & $\mathrm{N}^{14} \mathrm{H}_{3}$ \\
\hline 23,021 & & & $\mathrm{CH}_{3} \mathrm{NO}_{3}$ \\
\hline 23,033 & & - - & $\mathrm{SO}_{2}$ \\
\hline $23,046.10$ & M & - - - - & $\mathrm{N}^{15} \mathrm{H}_{3}$ \\
\hline $23,054.97$ & W & $\ldots-\ldots-\ldots$ & $\mathrm{N}^{15} \mathrm{H}_{3}$ \\
\hline $23,055.0$ & $\ldots$ & $1-2$ & $\mathrm{C}_{2} \mathrm{H}_{3} \mathrm{Cl}$ \\
\hline $23,098.78$ & $\mathrm{~S}$ & - - - - - - - & $\mathrm{N}^{14} \mathrm{H}_{3}$ \\
\hline 23,115 & & - & $\mathrm{CH}^{3} \mathrm{NH}_{2}$ \\
\hline $23,121.2$ & & & $\mathrm{CH}_{3} \mathrm{OH}$ \\
\hline 23,134 & M & - & $\mathrm{C}_{2} \mathrm{H}_{4} \mathrm{O}$ \\
\hline 23,181 & & & $\mathrm{CH}_{2} \mathrm{CF}_{2}$ \\
\hline $23,198.66$ & W & $1-2$ & $\mathrm{O}^{16} \mathrm{C}^{12} \mathrm{~S}^{36}$ \\
\hline 23,205 & & & $\mathrm{CH}_{3} \mathrm{NH}_{2}$ \\
\hline 23,206 & W & - & $\mathrm{CH}_{2} \mathrm{CF}_{2}$ \\
\hline 23,214 & M & - - - & $\mathrm{CH}_{2} \mathrm{CF}_{2}$ \\
\hline 23,220 & M & - & $\mathrm{CH}_{2} \mathrm{CF}_{2}$ \\
\hline $23,232.20$ & $\mathrm{~S}$ & 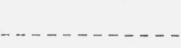 & $\mathrm{N}^{14} \mathrm{H}_{3}$ \\
\hline 23,234 & W & - & $\mathrm{CH}_{2} \mathrm{CF}_{2}$ \\
\hline 23,250 & $\ldots$ & $--1-1--1$ & $\mathrm{CH}_{3} \mathrm{NO}_{2}$ \\
\hline
\end{tabular}


TABLE 2. Lines listed by frequency-Continued

\begin{tabular}{|c|c|c|c|}
\hline Frequency & $\begin{array}{l}\text { Inten- } \\
\text { sity }\end{array}$ & Transition & Molecule \\
\hline $\begin{array}{l}23,295 \\
23,300\end{array}$ & $-\ldots$ & $-\cdots$ & $\begin{array}{l}\mathrm{CH}_{3} \mathrm{NH}_{2} \\
\mathrm{CH}_{3} \mathrm{NH}_{2}\end{array}$ \\
\hline 23,305 & & & $\mathrm{CH}_{3} \mathrm{NH}_{2}$ \\
\hline 23,308 & - & $\ldots$ & $\mathrm{CHClF}_{2}$ \\
\hline 23,323 & M & $\ldots$ & $\mathrm{CH}_{2} \mathrm{CF}_{2}$ \\
\hline 23,335 & M & $\ldots$ & $\mathrm{CH}_{3} \mathrm{NH}_{2}$ \\
\hline $23,346.8$ & - . . . & . . . . . & $\mathrm{CH}_{3} \mathrm{OH}$ \\
\hline 23,361 & M & - n. - n & $\mathrm{CH}_{2} \mathrm{CF}_{2}$ \\
\hline $23,372.72$ & M & $1-2$ & $\mathrm{Cl}^{37} \mathrm{C}^{12} \mathrm{~N}^{14}$ \\
\hline $23,389.00$ & M & $1-2$ & $\mathrm{Cl}^{37} \mathrm{C}^{12} \mathrm{~N}^{14}$ \\
\hline 23,389 & $\ldots$ & $1-2$ & $\mathrm{HN}^{14} \mathrm{C}^{13} \mathrm{~S}^{32}$ \\
\hline $23,389.61$ & M & $1-2$ & $\mathrm{Cl}^{37} \mathrm{C}^{12} \mathrm{~N}^{14}$ \\
\hline $23,390.53$ & M & $1-2$ & $\mathrm{Cl}^{37} \mathrm{C}^{12} \mathrm{~N}^{14}$ \\
\hline $23,402.47$ & M & $1-2$ & $\mathrm{Cl}^{37} \mathrm{C}^{12} \mathrm{~N}^{14}$ \\
\hline 23,404 & - . - . - & 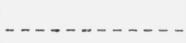 & $\mathrm{CH}_{3} \mathrm{NH}_{2}$ \\
\hline 23,413 & $\ldots \ldots$ & $-\ldots-n-1$ & $\mathrm{SO}_{2}$ \\
\hline $23,421.99$ & M & $\ldots-\ldots$ & $\mathrm{N}^{15} \mathrm{H}_{3}$ \\
\hline 23,425 & - - - - & $\ldots$ & $\mathrm{CH}_{3} \mathrm{OH}, \mathrm{CH}_{3} \mathrm{NH}_{2}$ \\
\hline 23,430 & $\ldots \ldots$ & - n & $\mathrm{CH}_{3} \mathrm{NH}_{2}$ \\
\hline 23,433 & $\mathrm{~S}$ & - $\ldots \ldots-\ldots-1$ & $\mathrm{CH}_{2} \mathrm{CF}_{2}$ \\
\hline 23,446 & - . - & - - & $\mathrm{CH}_{3} \mathrm{NH}_{2}$ \\
\hline $23,458.6$ & W & $1-2, K=0$ & $\mathrm{AsF}_{3}$ \\
\hline $23,463.0$ & W & $1-2, K=0$ & $\mathrm{AsF}_{3}$ \\
\hline 23,464 & $\ldots \ldots$ & $1-2$ & $\mathrm{HN}^{14} \mathrm{C}^{12} \mathrm{~S}^{32}$ \\
\hline 23,467 & $\ldots-\ldots$ & - $-\ldots-n-1$ & $\mathrm{CH}_{3} \mathrm{NO}_{2}$ \\
\hline $23,472.6$ & W & $1-2, K=1$ & $\mathrm{AsF}_{3}$ \\
\hline 23,476 & $\ldots \ldots$ & - & $\mathrm{CH}_{2} \mathrm{Cl}_{2}$ \\
\hline 23,483 & $\ldots \ldots$ & $-\ldots-n-\ldots$ & $\mathrm{CH}_{3} \mathrm{NO}_{2}$ \\
\hline $23,494.2$ & W & $1-2, K=1$ & $\mathrm{AsF}_{3}$ \\
\hline $23,501.6$ & W & $1-2, K=1$ & $\mathrm{AsF}_{3}$ \\
\hline 23,510 & & - & $\mathrm{CH}_{3} \mathrm{NH}_{2}$ \\
\hline $23,512.9$ & W & $\ldots \ldots \ldots$ & $\mathrm{AsF}_{3}$ \\
\hline $23,517.0$ & W & $1-2, K=0$ & $\mathrm{AsF}_{3}$ \\
\hline $23,522.4$ & M & $1-2, K=0$ & $\mathrm{AsF}_{3}$ \\
\hline $23,532.1$ & M & $1-2, K=1$ & $\mathrm{AsF}_{3}$ \\
\hline $23,534.67$ & W & $1-2$ & $\mathrm{O}^{17} \mathrm{C}^{12} \mathrm{~S}^{32}$ \\
\hline $23,538.9$ & -- & $1-2$ & $\mathrm{C}_{2} \mathrm{H}_{3} \mathrm{Cl}$ \\
\hline $23,543.2$ & W & - & $\mathrm{AsF}_{3}$ \\
\hline $23,553.0$ & W & 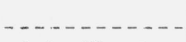 & $\mathrm{AsF}_{3}$ \\
\hline $23,564.6$ & W & $1-2, K=0$ & $\mathrm{AsF}_{3}$ \\
\hline $23,575.3$ & W & $1-2, K=1$ & $\mathrm{AsF}_{3}$ \\
\hline 23,610 & M & - - & $\mathrm{C}_{2} \mathrm{H}_{4} \mathrm{O}$ \\
\hline 23,644 & & $-\ldots-\ldots-\ldots$ & $\mathrm{CHClF}_{2}$ \\
\hline $23,646.92$ & W & $1-2, v=0$ & $\mathrm{O}^{16} \mathrm{C}^{13} \mathrm{C}^{34}$ \\
\hline 23,649 & M & $\ldots-\ldots$ & $\mathrm{CH}_{2} \mathrm{CF}_{2}$ \\
\hline $23,657.46$ & $\mathrm{~S}$ & $-\ldots-\ldots-n$ & $\mathrm{~N}^{14} \mathrm{H}_{3}$ \\
\hline 23,661 & W & $1-2, v_{1}=1$ & $\mathrm{O}^{16} \mathrm{C}^{12} \mathrm{~S}^{34}$ \\
\hline 23,680 & 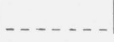 & - n & $\mathrm{CHClF}_{2}, \mathrm{CH}_{3} \mathrm{NH}_{2}$ \\
\hline
\end{tabular}

TABle 2. Lines listed by frequency-Continued

\begin{tabular}{|c|c|c|c|}
\hline Frequency & $\begin{array}{l}\text { Inten- } \\
\text { sity }\end{array}$ & Transition & Molecule \\
\hline $23,694.48$ & $\mathrm{~S}$ & & $\mathrm{~N}^{14} \mathrm{H}_{3}$ \\
\hline 23,706 & -1 & $\ldots$ & $\mathrm{CH}_{3} \mathrm{NO}_{2}$ \\
\hline $23,722.61$ & $\mathrm{~S}$ & $\ldots \ldots$ & $\mathrm{N}^{14} \mathrm{H}_{3}$ \\
\hline $23,731.33$ & M & $1-2, v=0$ & $\mathrm{O}^{16} \mathrm{C}^{12} \mathrm{~S}^{34}$ \\
\hline 23,733 & & $\ldots-\ldots$ & $\mathrm{CHClF}_{2}$ \\
\hline 23,738 & & - & $\mathrm{SO}_{2}$ \\
\hline $23,760.67$ & W & $1-2, v_{2}=1$ & $\mathrm{O}^{16} \mathrm{C}^{12} \mathrm{~S}^{34}$ \\
\hline 23,770 & $\mathrm{M}$ & - - & $\mathrm{CH}_{2} \mathrm{CF}_{2}$ \\
\hline $23,784.95$ & $\mathrm{~W}$ & $1-2, v_{2}=1$ & $\mathrm{O}^{16} \mathrm{C}^{12} \mathrm{~S}^{34}$ \\
\hline 23,803 & & - & $\mathrm{CHClF}_{2}$ \\
\hline 23,812 & $\mathrm{M}$ & $\ldots$ & $\mathrm{CH}_{2} \mathrm{CF}_{2}$ \\
\hline 23,826 & & $\ldots$ & $\mathrm{CHClF}_{2}$ \\
\hline 23,845 & & - - - & $\mathrm{CHClF}_{2}$ \\
\hline $23,854.4$ & $\ldots$ & $-\ldots$ & $\mathrm{CH}_{3} \mathrm{OH}$ \\
\hline $23,862.57$ & $\mathrm{M}$ & $1-2, v=0$ & $\mathrm{Cl}^{35} \mathrm{C}^{12} \mathrm{~N}^{14}$ \\
\hline $23,863.5$ & W & $1-2, v=0$ & $\mathrm{Cl}^{35} \mathrm{C}^{12} \mathrm{~N}^{4}$ \\
\hline $23,863.8$ & W & $1-2, v=0$ & $\mathrm{C} l^{35} \mathrm{C}^{12} \mathrm{~N}^{14}$ \\
\hline $23,864.0$ & M & $1-2, v=0$ & $\mathrm{Cl}^{35} \mathrm{C}^{12} \mathrm{~N}^{14}$ \\
\hline $23,864.2$ & M & $1-2, v=0$ & $\mathrm{C} l^{35} \mathrm{C}^{14} \mathrm{~N}^{14}$ \\
\hline $23,864.5$ & $\mathrm{M}$ & $1-2, v=0$ & $\mathrm{Cl}^{35} \mathrm{C}^{12} \mathrm{~N}^{14}$ \\
\hline $23,864.9$ & W & $1-2, v=0$ & $\mathrm{Cl}^{35} \mathrm{C}^{12} \mathrm{~N}^{14}$ \\
\hline $23,865.1$ & W & $1-2, v=0$ & $\mathrm{Cl}^{35} \mathrm{C}^{12} \mathrm{~N}^{14}$ \\
\hline $23,870.11$ & $\mathrm{~S}$ & - n & $\mathrm{N}^{14} \mathrm{H}_{3}$ \\
\hline $23,878.6$ & M & $1-2, v=0$ & $\mathrm{Cl}^{35} \mathrm{C}^{12} \mathrm{~N}^{14}$ \\
\hline $23,878.9$ & W & $1-2, v=0$ & $\mathrm{Cl}^{35} \mathrm{C}^{12} \mathrm{~N}^{14}$ \\
\hline $23,879.7$ & W & $1-2, v=0$ & $\mathrm{Cl}^{35} \mathrm{C}^{12} \mathrm{~N}^{14}$ \\
\hline $23,880.18$ & W & $2-3, v=0$ & $\mathrm{O}^{16} \mathrm{C}^{13} \mathrm{Se}^{80}$ \\
\hline $23,883.30$ & M & $1-2, v=0$ & $\mathrm{Cl}^{35} \mathrm{C}^{12} \mathrm{~N}^{14}$ \\
\hline $23,884.2$ & M & $1-2, v=0$ & $\mathrm{Cl}^{35} \mathrm{C}^{12} \mathrm{~N}^{14}$ \\
\hline $23,884.8$ & M & $1-2, v=0$ & $\mathrm{Cl}^{35} \mathrm{C}^{12} \mathrm{~N}^{14}$ \\
\hline $23,884.9$ & $\mathrm{~S}$ & $1-2, v=0$ & $\mathrm{Cl}^{35} \mathrm{C}^{12} \mathrm{~N}^{14}$ \\
\hline $23,885.16$ & $\mathrm{~S}$ & $1-2, v=0$ & $\mathrm{Cl}^{35} \mathrm{C}^{12} \mathrm{~N}^{14}$ \\
\hline $23,885.3$ & M & $1-2, v=0$ & $\mathrm{Cl}^{35} \mathrm{C}^{12} \mathrm{~N}^{14}$ \\
\hline $23,885.76$ & W & $1-2, v_{1}=1$ & $\mathrm{O}^{16} \mathrm{O}^{12} \mathrm{Se}^{82}$ \\
\hline $23,886.0$ & M & $1-2, v=0$ & $\mathrm{Cl}^{35} \mathrm{C}^{12} \mathrm{~N}^{14}$ \\
\hline $23,886.2$ & M & $1-2, v=0$ & $\mathrm{Cl}^{35} \mathrm{C}^{12} \mathrm{~N}^{14}$ \\
\hline $23,899.59$ & M & $1-2, v=0$ & $\mathrm{Cl}^{35} \mathrm{C}^{12} \mathrm{~N}^{14}$ \\
\hline $23,900.20$ & M & $1-2, v=0$ & $\mathrm{Cl}^{35} \mathrm{C}^{12} \mathrm{~N}^{14}$ \\
\hline $23,900.7$ & M & $1-2, v=0$ & $\mathrm{Cl}^{35} \mathrm{C}^{12} \mathrm{~N}^{14}$ \\
\hline $23,917.9$ & $\mathrm{~S}$ & $1-2, v_{2}=1$ & $\mathrm{Cl}^{35} \mathrm{C}^{12} \mathrm{~N}^{14}$ \\
\hline $23,920.91$ & M & $1-2, v=0$ & $\mathrm{Cl}^{35} \mathrm{C}^{12} \mathrm{~N}^{14}$ \\
\hline $23,922.32$ & M & - & $\mathrm{N}^{15} \mathrm{H}_{3}$ \\
\hline $23,925.5$ & M & $1-2, v_{2}=1$ & $\mathrm{Cl}{ }^{35} \mathrm{C}^{12} \mathrm{~N}^{14}$ \\
\hline $23,928.7$ & M & $1-2, v_{2}=1$ & $\mathrm{Cl}^{35} \mathrm{C}^{12} \mathrm{~N}^{14}$ \\
\hline $23,938.6$ & $\mathrm{~S}$ & $1-2, v_{2}=1$ & $\mathrm{Cl}^{35} \mathrm{C}^{12} \mathrm{~N}^{14}$ \\
\hline $23,944.4$ & M & $1-2, v_{2}=1$ & $\mathrm{Cl}^{35} \mathrm{C}^{12} \mathrm{~N}^{14}$ \\
\hline $23,948.2$ & $\mathrm{~S}$ & $1-2, v_{2}=1$ & $\mathrm{Cl}^{35} \mathrm{C}^{12} \mathrm{~N}^{14}$ \\
\hline
\end{tabular}


TABLE 2. Lines listed by frequency-Continued

\begin{tabular}{|c|c|c|c|}
\hline Frequency & $\begin{array}{l}\text { Inten- } \\
\text { sity }\end{array}$ & Transition & Molecule \\
\hline $23,954.5$ & M & $1-2, v_{2}=1$ & $\mathrm{Cl}^{35} \mathrm{C}^{12} \mathrm{~N}^{14}$ \\
\hline $23,958.4$ & M & $1-2, v_{2}=1$ & $\mathrm{Cl}^{35} \mathrm{C}^{12} \mathrm{~N}^{14}$ \\
\hline $23,964.50$ & M & $2-3, v=0$ & $\mathrm{O}^{16} \mathrm{C}^{12} \mathrm{Se}^{82}$ \\
\hline $23,968.6$ & $\mathrm{~S}$ & $1-2, v_{2}=1$ & $\mathrm{Cl}^{35} \mathrm{C}^{12} \mathrm{~N}^{14}$ \\
\hline $23,974.4$ & M & $1-2, v_{2}=1$ & $\mathrm{Cl}^{35} \mathrm{C}^{12} \mathrm{~N}^{14}$ \\
\hline $23,984.6$ & M & $1-2, v_{2}=1$ & $\mathrm{Cl}^{35} \mathrm{C}^{12} \mathrm{~N}^{14}$ \\
\hline 23,986 & M & $1-2, v_{2}=1$ & $\mathrm{CH}_{2} \mathrm{CF}_{2}$ \\
\hline $23,996.26$ & $\mathrm{~W}$ & $2-3, v_{2}=1$ & $\mathrm{O}^{16} \mathrm{C}^{12} \mathrm{Se}^{82}$ \\
\hline $24,013.04$ & W & $1-2, v=0$ & $\mathrm{O}^{16} \mathrm{C}^{12} \mathrm{~S}^{33}$ \\
\hline $24,014.97$ & $\mathrm{~W}$ & $2-3, v_{2}=1$ & $\mathrm{O}^{16} \mathrm{C}^{12} \mathrm{Se}^{82}$ \\
\hline 24,015 & $\ldots$ & $\ldots \ldots$ & $\mathrm{CH}_{3} \mathrm{NH}_{2}$ \\
\hline 24,017 & . & 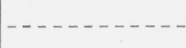 & $\mathrm{CH}_{3} \mathrm{NO}_{2}$ \\
\hline $24,020.21$ & W & $1-2, v=0$ & $\mathrm{O}^{16} \mathrm{C}^{12} \mathrm{~S}^{33}$ \\
\hline 24,021 & M & - n & $\mathrm{CH}_{2} \mathrm{CF}_{2}$ \\
\hline $24,025.39$ & $\mathrm{~W}$ & $1-2, v=0$ & $\mathrm{O}^{16} \mathrm{C}^{12} \mathrm{~S}^{33}$ \\
\hline $24,026.39$ & W & $2-3, v_{1}=1$ & $\mathrm{O}^{16} \mathrm{C}^{12} \mathrm{Se}^{80}$ \\
\hline $24,030.58$ & W & $2-3, v=0$ & $\mathrm{O}^{16} \mathrm{C}^{13} \mathrm{Se}^{78}$ \\
\hline $24,032.75$ & W & $1-2, v=0$ & $\mathrm{O}^{16} \mathrm{C}^{12} \mathrm{~S}^{33}$ \\
\hline 24,037 & & - & $\mathrm{SO}_{2}$ \\
\hline 24,055 & & - & $\mathrm{C}^{12} \mathrm{D}_{4} \mathrm{O}^{16}$ \\
\hline 24,078 & $\ldots$ & - & $\mathrm{CH}_{3} \mathrm{NH}_{2}$ \\
\hline 24,083 & & - & $\mathrm{SO}_{2}$ \\
\hline $24,105.98$ & M & $2-3, v=0$ & $\mathrm{O}^{16} \mathrm{C}^{12} \mathrm{Se}^{80}$ \\
\hline $24,138.05$ & M & $2-3, v_{2}=1$ & $\mathrm{O}^{16} \mathrm{C}^{12} \mathrm{Se}^{80}$ \\
\hline $24,139.45$ & $\mathrm{~S}$ & - & $\mathrm{N}^{14} \mathrm{H}_{3}$ \\
\hline 24,143 & & $4-5$ & $\mathrm{C}^{13} \mathrm{H}_{3} \mathrm{~N}^{14} \mathrm{C}^{12} \mathrm{~S}^{32}$ \\
\hline 24,150 & W & & $\mathrm{CH}_{2} \mathrm{CF}_{2}$ \\
\hline $24,156.93$ & M & $2-3, v_{2}=1$ & $\mathrm{O}^{16} \mathrm{C}^{12} \mathrm{Se}^{80}$ \\
\hline $24,173.0$ & W & $1-2, v=0$ & $\mathrm{O}^{16} \mathrm{C}^{14} \mathrm{~S}^{32}$ \\
\hline $24,174.30$ & W & $2-3, v_{1}=1$ & $\mathrm{O}^{16} \mathrm{C}^{12} \mathrm{Se}^{78}$ \\
\hline $24,205.25$ & $\mathrm{~S}$ & $\ldots$ & $\mathrm{N}^{14} \mathrm{H}_{3}$ \\
\hline $24,247.76$ & M & $1-2, v=0$ & $\mathrm{O}^{16} \mathrm{C}^{13} \mathrm{~S}^{32}$ \\
\hline $24,250.84$ & W & $2-3, v_{1}=1$ & $\mathrm{O}^{16} \mathrm{C}^{12} \mathrm{Se}^{77}$ \\
\hline $24,253.44$ & M & $1-2, v_{1}=1$ & $\mathrm{O}^{16} \mathrm{C}^{12} \mathrm{~S}^{32}$ \\
\hline $24,254.67$ & $\mathrm{M}$ & $2-3, v=0$ & $\mathrm{O}^{16} \mathrm{C}^{12} \mathrm{Se}^{78}$ \\
\hline $24,274.53$ & W & $0-1$ & $\mathrm{~N}^{15} \mathrm{~N}^{14} \mathrm{O}^{16}$ \\
\hline $24,274.61$ & W & $0-1$ & $\mathrm{~N}^{15} \mathrm{~N}^{14} \mathrm{O}^{16}$ \\
\hline $24,274.73$ & W & $0-1$ & $\mathrm{~N}^{15} \mathrm{~N}^{14} \mathrm{O}^{16}$ \\
\hline $24,274.78$ & W & $0-1$ & $\mathrm{~N}^{15} \mathrm{~N}^{15} \mathrm{O}^{16}$ \\
\hline $24,275.25$ & W & $1-2, v_{2}=1$ & $\mathrm{O}^{16} \mathrm{C}^{13} \mathrm{~S}^{32}$ \\
\hline $24,286.82$ & W & $2-3, v_{2}=1$ & $\mathrm{O}^{16} \mathrm{C}^{12} \mathrm{Se}^{78}$ \\
\hline 24,294 & M & - - - - - - & $\mathrm{CH}_{2} \mathrm{CF}_{2}$ \\
\hline $24,301.05$ & W & $1-2, v_{2}=1$ & $\mathrm{O}^{16} \mathrm{C}^{13} \mathrm{~S}^{32}$ \\
\hline $24,305.95$ & $\mathrm{~W}$ & $2-3, v_{2}=1$ & $\mathrm{O}^{16} \mathrm{C}^{12} \mathrm{Se}^{78}$ \\
\hline 24,320 & 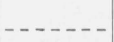 & - - - & $\mathrm{CH}_{3} \mathrm{NH}_{2}$ \\
\hline 24,323 & $\mathrm{~S}$ & $-\ldots-1-1$ & $\mathrm{CH}_{2} \mathrm{CF}_{2}$ \\
\hline $24,325.92$ & $\mathrm{~S}$ & $1-2, v=2$ & $\mathrm{O}^{16} \mathrm{C}^{12} \mathrm{~S}^{32}$ \\
\hline
\end{tabular}

Table 2. Lines listed by frequency-Continued

\begin{tabular}{|c|c|c|c|}
\hline Frequency & $\begin{array}{l}\text { Inten- } \\
\text { sity }\end{array}$ & Transition & Molecule \\
\hline $24,331.71$ & M & $2-3, v=0$ & $\mathrm{O}^{16} \mathrm{C}^{12} \mathrm{Se}^{77}$ \\
\hline 24,342 & & - & $\mathrm{SO}_{2}$ \\
\hline 24,352 & M & - - - - n - n & $\mathrm{CH}_{2} \mathrm{CF}_{2}$ \\
\hline $24,355.50$ & M & $1-2, v_{2}=1$ & $\mathrm{O}^{16} \mathrm{C}^{12} \mathrm{~S}^{32}$ \\
\hline 24,357 & W & - & $\mathrm{CH}_{2} \mathrm{CF}_{2}$ \\
\hline $24,363.97$ & W & $2-3, v_{2}=1$ & $\mathrm{O}^{16} \mathrm{C}^{12} \mathrm{Se}^{77}$ \\
\hline $24,380.84$ & M & $1-2, v_{2}=1$ & $\mathrm{O}^{16} \mathrm{C}^{12} \mathrm{~S}^{32}$ \\
\hline $24,383.21$ & W & $2-3, v_{2}=1$ & $\mathrm{O}^{16} \mathrm{C}^{12} \mathrm{Se}^{77}$ \\
\hline 24,401 & M & $2-3, v_{2}=2$ & $\mathrm{O}^{16} \mathrm{C}^{12} \mathrm{~S}^{32}$ \\
\hline $24,410.70$ & M & $2-3, v=0$ & $\mathrm{O}^{16} \mathrm{C}^{12} \mathrm{Se}^{76}$ \\
\hline 24,411 & W & $1-2, v=3$ & $\mathrm{O}^{16} \mathrm{C}^{12} \mathrm{~S}^{22}$ \\
\hline 24,428 & & $\ldots \ldots$ & $\mathrm{CH}_{3} \mathrm{NO}_{2}$ \\
\hline 24,440 & & - & $\mathrm{CH}_{2} \mathrm{Cl}_{2}$ \\
\hline $24,442.98$ & W & $2-3, v_{2}=1$ & $\mathrm{O}^{16} \mathrm{C}^{12} \mathrm{Se}^{76}$ \\
\hline 24,448 & & - & $\mathrm{CH}_{3} \mathrm{NO}_{2}$ \\
\hline 24,450 & $\mathrm{~S}$ & - & $\mathrm{CH}_{2} \mathrm{CF}_{2}$ \\
\hline 24,459 & W & $1-2, v_{2}=3$ & $\mathrm{O}^{16} \mathrm{C}^{12} \mathrm{~S}^{32}$ \\
\hline $24,462.42$ & W & $2-3, v_{2}=1$ & $\mathrm{O}^{16} \mathrm{C}^{12} \mathrm{Se}^{76}$ \\
\hline $24,465.33$ & M & $2-3, v=0$ & $\mathrm{Br}^{81} \mathrm{C}^{12} \mathrm{~N}^{14}$ \\
\hline 24,471 & & $\cdots$ & $\mathrm{CH}_{2} \mathrm{Cl}_{2}$ \\
\hline $24,506.75$ & M & $2-3, v_{1}=1$ & $\mathrm{Br}^{81} \mathrm{C}^{12} \mathrm{~N}^{14}$ \\
\hline $24,507.38$ & M & $2-3, v=0$ & $\mathrm{Br}^{81} \mathrm{C}^{12} \mathrm{~N}^{14}$ \\
\hline 24,528 & & $\ldots \ldots$ & $\mathrm{CH}_{3} \mathrm{NH}_{2}$ \\
\hline $24,532.94$ & $\mathrm{~S}$ & $\ldots$ & $\mathrm{N}^{14} \mathrm{H}_{3}$ \\
\hline $24,541.18$ & M & $2-3, v_{1}=1$ & $\mathrm{Br}^{81} \mathrm{C}^{12} \mathrm{~N}^{14}$ \\
\hline 24,545 & M & - & $\mathrm{CH}_{2} \mathrm{CF}_{2}$ \\
\hline $24,553.42$ & M & - - - & $\mathrm{N}^{15} \mathrm{H}_{3}$ \\
\hline $24,573.86$ & $\mathrm{~S}$ & $2-3, v=0$ & $\mathrm{Br}^{81} \mathrm{C}^{12} \mathrm{~N}^{14}$ \\
\hline $24,574.67$ & W & $2-3, v=0$ & $\mathrm{O}^{16} \mathrm{C}^{12} \mathrm{Se}^{74}$ \\
\hline 24,577 & & & $\mathrm{CH}_{2} \mathrm{Cl}_{2}$ \\
\hline 24,579 & W & $\ldots$ & $\mathrm{CH}_{2} \mathrm{CF}_{2}$ \\
\hline $24,583.00$ & M & $2-3, v=0$ & $\mathrm{Br}^{79} \mathrm{C}^{12} \mathrm{~N}^{14}$ \\
\hline 24,599 & & - & $\mathrm{CH}_{3} \mathrm{NO}_{2}$ \\
\hline 24,602 & W & - & $\mathrm{CH}_{2} \mathrm{CF}_{2}$ \\
\hline $24,608.92$ & $\mathrm{~S}$ & $2-3, v=0$ & $\mathrm{Br}^{81} \mathrm{C}^{12} \mathrm{~N}^{14}$ \\
\hline 24,609 & & $4-5$ & $\mathrm{C}^{12} \mathrm{H}_{3} \mathrm{~N}^{14} \mathrm{C}^{12} \mathrm{~S}^{34}$ \\
\hline $24,622.93$ & M & $2-3, v_{2}=1$ & $\mathrm{Br}^{81} \mathrm{C}^{12} \mathrm{~N}^{14}$ \\
\hline 24,627 & & - & $\mathrm{CH}_{2} \mathrm{Cl}_{2}$ \\
\hline 24,631 & $\ldots$ & - & $\mathrm{CH}_{2} \mathrm{Cl}_{2}$ \\
\hline $24,633.71$ & M & $2-3, v=0$ & $\mathrm{Br}^{79} \mathrm{C}^{12} \mathrm{~N}^{14}$ \\
\hline 24,636 & & $-1-1$ & $\mathrm{CH}_{2} \mathrm{Cl}_{2}$ \\
\hline 24,639 & M & - & $\mathrm{CH}_{2} \mathrm{CF}_{2}$ \\
\hline $24,645.82$ & M & $2-3, v_{1}=1$ & $\mathrm{Br}^{79} \mathrm{C}^{12} \mathrm{~N}^{14}$ \\
\hline $24,658.89$ & M & $2-3, v_{2}=1$ & $\mathrm{Br}^{81} \mathrm{C}^{12} \mathrm{~N}^{14}$ \\
\hline 24,668 & & - $-\ldots-\ldots$ & $\mathrm{C}_{2}{ }^{12} \mathrm{D}_{4} \mathrm{O}^{16}$ \\
\hline $24,682.13$ & M & $2-3, v_{2}=1$ & $\mathrm{Br}^{81} \mathrm{C}^{12} \mathrm{~N}^{14}$ \\
\hline $24,687.11$ & M & $2-3, v_{1}=1$ & $\mathrm{Br}^{79} \mathrm{C}^{12} \mathrm{~N}^{14}$ \\
\hline
\end{tabular}


TABLe 2. Lines listed by frequency-Continued

\begin{tabular}{|c|c|c|c|}
\hline Frequency & $\begin{array}{l}\text { Inten- } \\
\text { sity }\end{array}$ & Transition & Molecule \\
\hline $24,713.05$ & $\mathrm{~S}$ & $2-3, v=0$ & $\mathrm{Br}^{79} \mathrm{C}^{12} \mathrm{~N}^{14}$ \\
\hline $24,717.19$ & M & $2-3, v=0$ & $\mathrm{Br}^{81} \mathrm{C}^{12} \mathrm{~N}^{14}$ \\
\hline 24,734 & M & - - - & $\mathrm{CH}_{2} \mathrm{CF}_{2}$ \\
\hline $24,755.22$ & $\mathrm{~S}$ & $2-3, v=0$ & $\mathrm{Br}^{79} \mathrm{C}^{12} \mathrm{~N}^{14}$ \\
\hline $24,760.76$ & M & $2-3, v_{2}=1$ & $\mathrm{Br}^{79} \mathrm{C}^{12} \mathrm{~N}^{14}$ \\
\hline 24,770 & S & $\ldots$ & $\mathrm{CH}_{2} \mathrm{CF}_{2}$ \\
\hline $24,784.02$ & M & $2-3, v_{2}=1$ & $\mathrm{Br}^{79} \mathrm{C}^{12} \mathrm{~N}^{14}$ \\
\hline $24,803.00$ & $\mathrm{M}$ & $2-3, v_{2}=1$ & $\mathrm{Br}^{79} \mathrm{C}^{12} \mathrm{~N}^{14}$ \\
\hline 24,806 & W & $\ldots \ldots$ & $\mathrm{CH}_{2} \mathrm{CF}_{2}$ \\
\hline 24,808 & W & - & $\mathrm{CH}_{2} \mathrm{CF}_{2}$ \\
\hline 24,824 & $\ldots$ & $4-5 \ldots \ldots$ & $\mathrm{C}^{12} \mathrm{H}_{3} \mathrm{~N}^{14} \mathrm{C}^{12} \mathrm{~S}^{34}$ \\
\hline $24,826.70$ & M & $2-3, v_{2}=1$ & $\mathrm{Br}^{79} \mathrm{C}^{12} \mathrm{~N}^{14}$ \\
\hline $24,834.3$ & $\mathrm{~S}$ & - - - - - & $\mathrm{C}_{2} \mathrm{H}_{4} \mathrm{O}$ \\
\hline 24,842 & & - & $\mathrm{CH}_{2} \mathrm{Cl}_{2}$ \\
\hline $24,860.6$ & M & $2-3, v_{2}=2$ & $\mathrm{Br}^{79} \mathrm{C}^{12} \mathrm{~N}^{14}$ \\
\hline $24,881.90$ & $\mathrm{~S}$ & - & $\mathrm{N}^{14} \mathrm{H}_{3}$ \\
\hline $24,884.57$ & M & $2-3, v=0$ & $\mathrm{Br}^{79} \mathrm{C}^{12} \mathrm{~N}^{14}$ \\
\hline $24,890.0$ & M & $2-3, v_{2}=1$ & $\mathrm{Br}^{79} \mathrm{C}^{12} \mathrm{~N}^{14}$ \\
\hline 24,890 & & . & $\mathrm{CH}_{3} \mathrm{NH}_{2}$ \\
\hline 24,896 & - & $\ldots$ & $\mathrm{CH}_{3} \mathrm{NH}_{2}$ \\
\hline 24,910 & $\ldots \ldots$ & $4-5$ & $\mathrm{C}^{12} \mathrm{H}_{3} \mathrm{~N}^{14} \mathrm{C}^{13} \mathrm{~S}^{34}$ \\
\hline 24,911 & $-\ldots$ & $\ldots$ & $\mathrm{CH}_{3} \mathrm{OH}$ \\
\hline 24,916 & 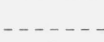 & - & $\mathrm{CH}_{2} \mathrm{Cl}_{2}$ \\
\hline $24,924.4$ & $\mathrm{~S}$ & & $\mathrm{C}_{2} \mathrm{H}_{4} \mathrm{O}$ \\
\hline 24,926 & - & $\ldots$ & $\mathrm{CH}_{3} \mathrm{OH}$ \\
\hline $24,928.70$ & & & $\mathrm{CH}_{3} \mathrm{OH}$ \\
\hline 24,930 & - & $4-5$ & $\mathrm{C}^{12} \mathrm{H}_{3} \mathrm{~N}^{14} \mathrm{C}^{12} \mathrm{~S}^{33}$ \\
\hline $24,933.47$ & & $\ldots$ & $\mathrm{CH}_{3} \mathrm{OH}$ \\
\hline $24,934.38$ & & & $\mathrm{CH}_{3} \mathrm{OH}$ \\
\hline $24,959.08$ & & & $\mathrm{CH}_{3} \mathrm{OH}$ \\
\hline 24,967 & & & $\mathrm{CH}_{2} \mathrm{Cl}_{2}$ \\
\hline 24,970 & & & $\mathrm{CH}_{2} \mathrm{Cl}_{2}$ \\
\hline 24,971 & & $4-5$ & $\mathrm{C}^{12} \mathrm{H}_{3} \mathrm{~N}^{14} \mathrm{C}^{12} \mathrm{~S}^{32}$ \\
\hline 24,976 & & & $\mathrm{CH}_{2} \mathrm{Cl}_{2}$ \\
\hline $24,981.5$ & M & $2-3, v_{2}=2$ & $\mathrm{Br}^{79} \mathrm{C}^{12} \mathrm{~N}^{14}$ \\
\hline 24,996 & & $\ldots$ & $\mathrm{CH}_{2} \mathrm{Cl}_{2}$ \\
\hline 25,000 & - & $\ldots \ldots$ & $\mathrm{CH}_{2} \mathrm{NH}_{2}$ \\
\hline 25,002 & & $4-5$ & $\mathrm{C}^{12} \mathrm{H}_{3} \mathrm{~N}^{14} \mathrm{C}^{13} \mathrm{~S}^{32}$ \\
\hline $25,006.0$ & M & $2-3, v_{2}=1$ & $\mathrm{Br}^{79} \mathrm{C}^{12} \mathrm{~N}^{14}$ \\
\hline 25,018 & & & $\mathrm{CH}_{2} \mathrm{Cl}_{2}$ \\
\hline $25,018.14$ & & & $\mathrm{CH}_{3} \mathrm{OH}$ \\
\hline 25,038 & 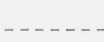 & - . . . - & $\mathrm{CH}_{2} \mathrm{Cl}_{2}$ \\
\hline 25,046 & & & $\mathrm{CH}_{2} \mathrm{Cl}_{2}$ \\
\hline 25,047 & & & $\mathrm{CH}_{2} \mathrm{Cl}_{2}$ \\
\hline 25,053 & & & $\mathrm{CH}_{2} \mathrm{Cl}_{2}, \mathrm{CH}_{3} \mathrm{OH}$ \\
\hline 25,056 & & & $\mathrm{CH}_{2} \mathrm{Br}_{2}$ \\
\hline $25,056.04$ & $\mathrm{~S}$ & - - - - - - - & $\mathrm{N}^{14} \mathrm{H}_{3}$ \\
\hline 25,065 & 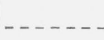 & & $\mathrm{CH}_{3} \mathrm{NH}_{2}$ \\
\hline
\end{tabular}

Table 2. Lines listed by frequency-Continued

\begin{tabular}{|c|c|c|c|}
\hline Frequency & $\begin{array}{l}\text { Inten- } \\
\text { sity }\end{array}$ & Transition & Molecule \\
\hline 25,073 & & & $\mathrm{CH}_{2} \mathrm{Cl}_{2}$ \\
\hline 25,077 & & $4-5$ & $\mathrm{C}^{12} \mathrm{H}_{3} \mathrm{~N}^{14} \mathrm{C}^{12} \mathrm{~S}^{32}$ \\
\hline 25,099 & - & 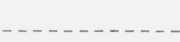 & $\mathrm{CH}_{2} \mathrm{Cl}_{2}$ \\
\hline 25,100 & & $4-5$ & $\mathrm{C}^{12} \mathrm{H}_{3} \mathrm{~N}^{14} \mathrm{C}^{12} \mathrm{~S}^{32}$ \\
\hline $25,121.55$ & W & $0-1$ & $\mathrm{~N}^{14} \mathrm{~N}^{15} \mathrm{O}^{16}$ \\
\hline 25,123 & & & $\mathrm{CH}_{2} \mathrm{Cl}_{2}$ \\
\hline $25,123.03$ & M & $0-1$ & $\mathrm{~N}_{2}{ }^{14} \mathrm{O}^{16}$ \\
\hline $25,123.28$ & M & $0-1$ & $\mathrm{~N}_{2}{ }^{14} \mathrm{O}^{16}$ \\
\hline $25,123.64$ & W & $0-1$ & $\mathrm{~N}_{2}{ }^{14} \mathrm{O}^{16}$ \\
\hline $25,124.88$ & & --- & $\mathrm{CH}_{3} \mathrm{OH}$ \\
\hline 25,161 & 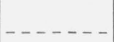 & $4-5$ & $\mathrm{C}^{12} \mathrm{H}_{3} \mathrm{~N}^{14} \mathrm{C}^{12} \mathrm{~S}^{32}$ \\
\hline $25,167.4$ & W & $0-1, K=0$ & $\mathrm{C}^{13} \mathrm{H}_{3} \mathrm{Cl}^{37}$ \\
\hline 25,170 & & - - - & $\mathrm{SO}_{2}$ \\
\hline $25,183.0$ & W & $0-1, K=0$ & $\mathrm{C}^{13} \mathrm{H}_{3} \mathrm{Cl}^{37}$ \\
\hline 25,195 & $\ldots$ & $4-5$ & $\mathrm{C}^{12} \mathrm{H}_{3} \mathrm{~N}^{14} \mathrm{C}^{12} \mathrm{~S}^{32}$ \\
\hline $25,195.2$ & W & $0-1, K=0$ & $\mathrm{C}^{13} \mathrm{H}_{3} \mathrm{Cl}^{37}$ \\
\hline 25,200 & $\ldots$ & - & $\mathrm{CH}_{3} \mathrm{NH}_{2}$ \\
\hline 25,221 & $\ldots$ & - - & $\mathrm{CH}_{2} \mathrm{Cl}_{2}$ \\
\hline 25,248 & M & --- & $\mathrm{CH}_{2} \mathrm{CF}_{2}$ \\
\hline 25,269 & & & $\mathrm{CH}_{2} \mathrm{Cl}_{2}$ \\
\hline 25,269 & & $4-5$ & $\mathrm{C}^{12} \mathrm{H}_{3} \mathrm{~N}^{14} \mathrm{C}^{12} \mathrm{~S}^{32}$ \\
\hline 25,280 & & $-\ldots$ & $\mathrm{CH}_{2} \mathrm{Cl}_{2}$ \\
\hline $25,294.41$ & - & $-\ldots$ & $\mathrm{CH}_{3} \mathrm{OH}$ \\
\hline 25,306 & & $4-5$ & $\mathrm{C}^{12} \mathrm{H}_{3} \mathrm{~N}^{14} \mathrm{C}^{12} \mathrm{~S}^{32}$ \\
\hline $25,323.51$ & M & & $\mathrm{N}^{15} \mathrm{H}_{3}$ \\
\hline 25,333 & & $4-5$ & $\mathrm{C}^{12} \mathrm{H}_{3} \mathrm{~N}^{14} \mathrm{C}^{12} \mathrm{~S}^{32}$ \\
\hline 25,350 & $\mathrm{~S}$ & & $\mathrm{CH}_{2} \mathrm{CF}_{2}$ \\
\hline 25,381 & & --- & $\mathrm{CH}_{3} \mathrm{OH}$ \\
\hline 25,390 & & $4-5$ & $\mathrm{C}^{12} \mathrm{H}_{3} \mathrm{~N}^{14} \mathrm{C}^{12} \mathrm{~S}^{32}$ \\
\hline 25,392 & & & $\mathrm{SO}_{2}$ \\
\hline $25,393.87$ & M & $3-4, v=0$ & $\mathrm{IC}^{12} \mathrm{~N}^{14}$ \\
\hline 25,404 & & $-\ldots-\ldots-1$ & $\mathrm{CH}_{3} \mathrm{NO}_{2}$ \\
\hline 25,409 & 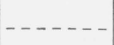 & $4-5$ & $\mathrm{C}^{12} \mathrm{H}_{3} \mathrm{~N}^{14} \mathrm{C}^{12} \mathrm{~S}^{32}$ \\
\hline 25,414 & & --- & $\mathrm{CH}_{3} \mathrm{NO}_{2}$ \\
\hline 25,442 & & $4-5$ & $\mathrm{C}^{12} \mathrm{H}_{3} \mathrm{~N}^{14} \mathrm{C}^{12} \mathrm{~S}^{32}$ \\
\hline 25,450 & M & - - - - & $\mathrm{CH}_{2} \mathrm{CF}_{2}$ \\
\hline 25,470 & & $\ldots-\ldots \ldots$ & $\mathrm{CH}_{3} \mathrm{NH}_{2}$ \\
\hline 25,516 & $\mathrm{~S}$ & $-\ldots-\ldots$. & $\mathrm{CH}_{2} \mathrm{CF}_{2}$ \\
\hline $25,541.43$ & 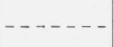 & $4-5$ & $\mathrm{CH}_{3} \mathrm{OH}$ \\
\hline 25,550 & & $4-5$ & $\mathrm{C}^{12} \mathrm{H}_{3} \mathrm{~N}^{14} \mathrm{C}^{12} \mathrm{~S}^{32}$ \\
\hline $25,577.2$ & W & $0-1, K=0$ & $\mathrm{C}^{13} \mathrm{H}^{3} \mathrm{Cl}^{35}$ \\
\hline $25,596.0$ & W & $0-1, K=0$ & $\mathrm{C}^{13} \mathrm{H}_{3} \mathrm{Cl}^{35}$ \\
\hline $25,611.2$ & W & $0-1, K=0$ & $\mathrm{C}^{13} \mathrm{H}^{3} \mathrm{Cl}^{35}$ \\
\hline 25,653 & $\ldots$ & $4-5$ & $\mathrm{C}^{12} \mathrm{H}_{3} \mathrm{~N}^{14} \mathrm{C}^{12} \mathrm{~S}^{32}$ \\
\hline $25,695.23$ & $\mathrm{~S}$ & - - - - & $\mathrm{N}^{14} \mathrm{H}_{3}$ \\
\hline 25,710 & $\ldots$ & - - - - & $\mathrm{CH}_{2} \mathrm{Cl}_{2}$ \\
\hline $25,711.50$ & $\mathrm{~S}$ & $3-4, v=0$ & $\mathrm{IC}^{12} \mathrm{~N}^{14}$ \\
\hline
\end{tabular}


Table 2. Lines listed by frequency-Continued

\begin{tabular}{|c|c|c|c|}
\hline Frequency & $\begin{array}{l}\text { Inten- } \\
\text { sity }\end{array}$ & Transition & Molecule \\
\hline $25,715.14$ & $\mathrm{~S}$ & & $\mathrm{~N}^{14} \mathrm{H}_{3}$ \\
\hline $25,728.77$ & $\mathrm{~S}$ & $3-4, v=0$ & $\mathrm{IC}^{12} \mathrm{~N}^{14}$ \\
\hline 25,729 & $\mathrm{~S}$ & - & $\mathrm{CH}_{2} \mathrm{CF}_{2}$ \\
\hline 25,741 & M & - & $\mathrm{CH}_{2} \mathrm{CF}_{2}$ \\
\hline 25,745 & - & - - & $\mathrm{CH}_{2} \mathrm{Cl}_{2}$ \\
\hline 25,748 & & & $\mathrm{CH}_{2} \mathrm{Cl}_{2}$ \\
\hline $25,748,18$ & M & $3-4, v_{1}=1$ & $\mathrm{IC}^{12} \mathrm{~N}^{14}$ \\
\hline 25,751 & $\ldots$ & 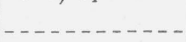 & $\mathrm{CH}_{2} \mathrm{Cl}_{2}$ \\
\hline $25,752.65$ & $\mathrm{~S}$ & $3-4, v=0$ & $\mathrm{IC}^{12} \mathrm{~N}^{14}$ \\
\hline $25,763.23$ & M & $3-4, v_{1}=1$ & $\mathrm{IC}^{12} \mathrm{~N}^{14}$ \\
\hline $25,783.50$ & $\mathrm{~S}$ & $3-4, v=0$ & $\mathrm{IC}^{12} \mathrm{~N}^{14}$ \\
\hline $25,789.85$ & $\mathrm{~S}$ & $3-4, v=0$ & $\mathrm{IC}^{12} \mathrm{~N}^{14}$ \\
\hline 25,797 & & $-\ldots-\ldots-\ldots$ & $\mathrm{CH}_{3} \mathrm{OH}$ \\
\hline $25,802.92$ & M & $3-4, v_{2}=1$ & $\mathrm{IC}^{12} \mathrm{~N}^{14}$ \\
\hline $25,815.34$ & M & $3-4, v_{2}=1$ & $\mathrm{IC}^{12} \mathrm{~N}^{14}$ \\
\hline $25,823.08$ & $\mathrm{~S}$ & $3-4, v=0$ & $\mathrm{IC}^{12} \mathrm{~N}^{14}$ \\
\hline $25,829.31$ & M & $3-4, v_{2}=1$ & $\mathrm{IC}^{12} \mathrm{~N}^{14}$ \\
\hline $25,837.64$ & $\mathrm{~S}$ & $3-4, v=0$ & $\mathrm{IC}^{12} \mathrm{~N}^{14}$ \\
\hline 25,848 & & $\ldots$ & $\mathrm{CH}_{2} \mathrm{Cl}_{2}$ \\
\hline $25,850.78$ & M & $3-4, v_{2}=1$ & $\mathrm{IC}^{12} \mathrm{~N}^{14}$ \\
\hline $25,872.24$ & $\mathrm{~S}$ & $3-4, v_{2}=1$ & $\mathrm{IC}^{12} \mathrm{~N}^{14}$ \\
\hline $25,878.18$ & & - & $\mathrm{CH}_{3} \mathrm{OH}$ \\
\hline $25,893.73$ & $\mathrm{~S}$ & $3-4, v_{2}=1$ & $\mathrm{IC}^{12} \mathrm{~N}^{14}$ \\
\hline $25,906.28$ & $\mathrm{~S}$ & $3-4, v_{2}=1$ & $\mathrm{IC}^{12} \mathrm{~N}^{14}$ \\
\hline $25,927.66$ & $\mathrm{~S}$ & $3-4, v_{2}=1$ & $\mathrm{IC}^{12} \mathrm{~N}^{14}$ \\
\hline $24,954.36$ & $\mathrm{~S}$ & $3-4, v=0$ & $\mathrm{IC}^{12} \mathrm{~N}^{14}$ \\
\hline $25,969.58$ & M & $3-4, v=0$ & $\mathrm{IC}^{12} \mathrm{~N}^{14}$ \\
\hline $25,991.92$ & $\mathrm{~S}$ & $3-4, v=0$ & $\mathrm{IC}^{12} \mathrm{~N}^{14}$ \\
\hline 26,007 & & $-\ldots$ & $\mathrm{CH}_{2} \mathrm{Cl}_{2}$ \\
\hline $26,042.41$ & W & $1-2, K=0$ & $\mathrm{SiH}_{3} \mathrm{Cl}^{37}$ \\
\hline $26,043.29$ & W & $1-2, K=1$ & $\mathrm{SiH}_{3} \mathrm{Cl}^{37}$ \\
\hline $26,046.30$ & W & $1-2, K=1$ & $\mathrm{SiH}_{3} \mathrm{Cl}^{37}$ \\
\hline $26,046.32$ & M & $3-4, v_{2}=2$ & $\mathrm{IC}^{12} \mathrm{~N}^{14}$ \\
\hline $26,047.97$ & W & $1-2, K=1$ & $\mathrm{SiH}_{3} \mathrm{Cl}^{37}$ \\
\hline $26,050.26$ & M & $1-2, K=0$ & $\mathrm{SiH}_{3} \mathrm{Cl}^{37}$ \\
\hline $26,051.09$ & W & $1-2, K=1$ & $\mathrm{SiH}_{3} \mathrm{Cl}^{37}$ \\
\hline $26,053.35$ & W & $1-2, K=1$ & $\mathrm{SiH}_{3} \mathrm{Cl}^{37}$ \\
\hline $26,055.86$ & W & $1-2, K=0$ & $\mathrm{SiH}_{3} \mathrm{Cl}^{37}$ \\
\hline $26,057.10$ & W & $1-2, K=1$ & $\mathrm{SiH}_{3} \mathrm{Cl}^{37}$ \\
\hline $26,063.52$ & W & $1-2, K=0$ & $\mathrm{SiH}_{3} \mathrm{Cl}^{37}$ \\
\hline 26,118 & $\mathrm{~S}$ & - - - & $\mathrm{CH}_{2} \mathrm{CF}_{2}$ \\
\hline 26,120 & --- & - - - - - - - & $\mathrm{CH}_{3} \mathrm{OH}$ \\
\hline 26,163 & M & $-\ldots-\ldots-n$ & $\mathrm{CH}_{2} \mathrm{CF}_{2}$ \\
\hline $26,164.57$ & M & $0-1, K=0$ & $\mathrm{CH}_{3} \mathrm{Cl}^{37}$ \\
\hline $26,179.30$ & M & $0-1, K=0$ & $\mathrm{CH}_{3} \mathrm{Cl}^{37}$ \\
\hline $26,181.6$ & M & $3-4, v=0$ & $\mathrm{ICl}^{37}$ \\
\hline $26,191.13$ & M & $0-1, K=0$ & $\mathrm{CH}_{3} \mathrm{Cl}^{37}$ \\
\hline
\end{tabular}

Taвle 2. Lines listed by frequency-Continued

\begin{tabular}{|c|c|c|c|}
\hline Frequency & $\begin{array}{l}\text { Inten- } \\
\text { sity }\end{array}$ & Transition & Molecule \\
\hline $\begin{array}{l}26,295 \\
26,310\end{array}$ & & & $\begin{array}{l}\mathrm{CH}_{2} \mathrm{Cl}_{2} \\
\mathrm{CH}_{3} \mathrm{OH}\end{array}$ \\
\hline 26,328 & $\mathrm{~S}$ & & $\mathrm{CH}_{2} \mathrm{CF}_{2}$ \\
\hline 26,337 & M & & $\mathrm{CH}_{2} \mathrm{CF}_{2}$ \\
\hline 26,410 & W & & $\mathrm{CH}_{2} \mathrm{CF}_{2}$ \\
\hline 26,466 & M & & $\mathrm{CH}_{2} \mathrm{CF}_{2}$ \\
\hline $26,518.91$ & $\mathrm{~S}$ & & $\mathrm{~N}^{14} \mathrm{H}_{3}$ \\
\hline 26,553 & $\ldots$ & & $\mathrm{SPF}_{3}$ \\
\hline 26,562 & & & $\mathrm{CH}_{3} \mathrm{OH}$ \\
\hline 26,565 & $-\ldots$ & $-\cdots$ & $\mathrm{C}^{12} \mathrm{D}_{4} \mathrm{O}^{16}$ \\
\hline $26,570.77$ & M & $0-1, K=0$ & $\mathrm{CH}_{3} \mathrm{Cl}^{35}$ \\
\hline 26,574 & $\ldots$ & $-\ldots \ldots-\ldots$ & $\mathrm{SPF}_{3}$ \\
\hline $26,589.49$ & M & $0-1, K=0$ & $\mathrm{CH}_{3} \mathrm{Cl}^{35}$ \\
\hline $26,604.57$ & M & $0-1, K=0$ & $\mathrm{CH}_{3} \mathrm{Cl}^{35}$ \\
\hline 26,634 & $\mathrm{~S}$ & & $\mathrm{CH}_{2} \mathrm{CF}_{2}$ \\
\hline 26,649 & M & - & $\mathrm{CH}_{2} \mathrm{CF}_{2}$ \\
\hline $26,655.00$ & $\mathrm{~S}$ & - & $\mathrm{N}^{14} \mathrm{H}_{3}$ \\
\hline $26,685.25$ & W & $1-2, K=0$ & $\mathrm{SiH}_{3} \mathrm{Cl}^{35}$ \\
\hline $26,686.15$ & W & $1-2, K=0$ & $\mathrm{SiH}_{3} \mathrm{Cl}^{35}$ \\
\hline $26,687.34$ & W & $1-2, K=1$ & $\mathrm{SiH}_{3} \mathrm{Cl}^{35}$ \\
\hline $26,690.92$ & W & $1-2, K=1$ & $\mathrm{SiH}_{3} \mathrm{Cl}^{35}$ \\
\hline $26,692.66$ & W & $1-2, K=1$ & $\mathrm{SiH}_{3} \mathrm{Cl}^{35}$ \\
\hline $26,695.00$ & W & $1-2, K=0$ & $\mathrm{SiH}_{3} \mathrm{Cl}^{35}$ \\
\hline $26,696.10$ & M & $1-2, K=0$ & $\mathrm{SiH}_{3} \mathrm{Cl}^{35}$ \\
\hline $26,697.45$ & M & $1-2, K=1$ & $\mathrm{SiH}_{3} \mathrm{Cl}^{35}$ \\
\hline $26,700.48$ & W & $1-2, K=1$ & $\mathrm{SiH}_{3} \mathrm{Cl}^{35}$ \\
\hline $26,703.34$ & W & $1-2, K=0$ & $\mathrm{SiH}_{3} \mathrm{Cl}^{35}$ \\
\hline $26,705.02$ & W & $1-2, K=1$ & $\mathrm{SiH}_{3} \mathrm{Cl}^{35}$ \\
\hline $26,713.07$ & W & $1-2, K=0$ & $\mathrm{SiH}_{3} \mathrm{Cl}^{35}$ \\
\hline 26,723 & W & & $\mathrm{CH}_{2} \mathrm{CF}_{2}$ \\
\hline 26,832 & M & - . - - - & $\mathrm{CH}_{2} \mathrm{CF}_{2}$ \\
\hline 26,865 & $\mathrm{~S}$ & $\ldots$ & $\mathrm{CH}_{2} \mathrm{CF}_{2}$ \\
\hline 26,880 & M & $-\ldots \ldots$ & $\mathrm{CH}_{2} \mathrm{CF}_{2}$ \\
\hline 26,992 & $\mathrm{~S}$ & - . . . & $\mathrm{CH}_{2} \mathrm{CF}_{2}$ \\
\hline 27,014 & $\mathrm{~S}$ & & $\mathrm{CH}_{2} \mathrm{CF}_{2}$ \\
\hline 27,112 & M & - & $\mathrm{CH}_{2} \mathrm{CF}_{2}$ \\
\hline $27,194.75$ & M & $3-4, v=0$ & $\mathrm{ICl}^{35}$ \\
\hline $27,202.64$ & W & $3-4, v=0$ & $\mathrm{ICl}^{35}$ \\
\hline 27, 204. 99 & M & $3-4, v=0$ & $\mathrm{ICl}^{35}$ \\
\hline $27,208.54$ & M & $3-4, v=1$ & $1 \mathrm{Cl}^{35}$ \\
\hline 27,216 & $\mathrm{M}$ & - & $\mathrm{CH}_{2} \mathrm{CF}_{2}$ \\
\hline $27,217.51$ & M & $3-4, v=0$ & $\mathrm{ICl}^{35}$ \\
\hline $27,221.02$ & M & $3-4, v=0$ & $\mathrm{ICl}^{35}$ \\
\hline $27,225.32$ & M & $3-4, v=0$ & $\mathrm{ICl}^{35}$ \\
\hline $27,228.34$ & M & $3-4, v=0$ & $\mathrm{ICl}^{35}$ \\
\hline $27,242.59$ & W & $3-4, v=0$ & $\mathrm{ICl}^{35}$ \\
\hline $27,254.90$ & M & $3-4, v=0$ & $\mathrm{ICl}^{35}$ \\
\hline $27,283.66$ & $\ldots-\ldots$ & $3-4, v=0$ & $\mathrm{ICl}^{35}$ \\
\hline
\end{tabular}


TABLe 2. Lines listed by frequency-Continued

\begin{tabular}{|c|c|c|c|}
\hline Frequency & $\begin{array}{c}\text { Inten- } \\
\text { sity }\end{array}$ & Transition & Molecule \\
\hline $27,286.25$ & $\ldots$ & $3-4, v=0$ & $\mathrm{ICl}^{35}$ \\
\hline $27,292.63$ & $\ldots$ & $3-4, v=0$ & $\mathrm{ICl}^{35}$ \\
\hline $27,295.05$ & ..... & $3-4, v=0$ & $\mathrm{ICl}^{35}$ \\
\hline 27,297 & $\mathrm{~S}$ & - & $\mathrm{CH}_{2} \mathrm{CF}_{2}$ \\
\hline $27,333.85$ & M & $3-4, v=0$ & $\mathrm{ICl}^{35}$ \\
\hline $27,336.68$ & M & $3-4, v=0$ & $\mathrm{ICl}^{35}$ \\
\hline $27,337.38$ & M & $3-4, v=0$ & $\mathrm{ICl}^{35}$ \\
\hline $27,346.31$ & M & $3-4, v=0$ & $\mathrm{ICl}^{35}$ \\
\hline $27,354.71$ & M & $3-4, v=0$ & $\mathrm{ICl}^{35}$ \\
\hline $27,356.58$ & M & $3-4, v=0$ & $\mathrm{ICl}^{35}$ \\
\hline $27,357.73$ & M & $3-4, v=0$ & $\mathrm{ICl}^{35}$ \\
\hline 27,412 & M & - $\ldots \ldots$ & $\mathrm{CH}_{2} \mathrm{CF}_{2}$ \\
\hline 27,478 & $\mathrm{~S}$ & ... & $\mathrm{N}^{14} \mathrm{H}_{3}$ \\
\hline 27,681 & $\mathrm{~S}$ & - & $\mathrm{CH}_{2} \mathrm{CF}_{2}$ \\
\hline $27,772.52$ & M & - & $\mathrm{N}^{14} \mathrm{H}_{3}$ \\
\hline 27,818 & $\mathrm{~S}$ & 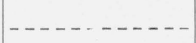 & $\mathrm{CH}_{2} \mathrm{CF}_{2}$ \\
\hline 27,972 & $\mathrm{~S}$ & - & $\mathrm{CH}_{2} \mathrm{CF}_{2}$ \\
\hline $28,069.99$ & W & $1-2, K=0$ & $\mathrm{C}^{\cdot 3} \mathrm{H}_{3} \mathrm{I}$ \\
\hline $28,145.01$ & W & $1-2, K=0$ & $\mathrm{C}^{13} \mathrm{H}_{3} \mathrm{I}$ \\
\hline 28,174 & $\mathrm{~S}$ & - & $\mathrm{CH}_{2} \mathrm{CF}_{2}$ \\
\hline $28,206.90$ & W & $1-2, K=1$ & $\mathrm{C}^{13} \mathrm{H}_{3} \mathrm{I}$ \\
\hline $28,253.84$ & W & $1-2, K=1$ & $\mathrm{C}^{13} \mathrm{H}_{3} \mathrm{I}$ \\
\hline 28,314 & W & -... & $\mathrm{CH}_{2} \mathrm{CF}_{2}$ \\
\hline 28,339 & M & - - - & $\mathrm{CH}_{2} \mathrm{CF}_{2}$ \\
\hline $28,343.64$ & W & $1-2, K=0$ & $\mathrm{C}^{13} \mathrm{H}_{3} \mathrm{I}$ \\
\hline 28,380 & $\ldots$ & $4-5$ & $\mathrm{C}^{12} \mathrm{H}_{3} \mathrm{~S}^{32} \mathrm{C}^{12} \mathrm{~N}^{14}$ \\
\hline $28,411.19$ & W & $1-2, K=1$ & $\mathrm{C}^{13} \mathrm{H}_{3} \mathrm{I}$ \\
\hline 28,412 & $\mathrm{~S}$ & - - & $\mathrm{CH}_{2} \mathrm{CF}_{2}$ \\
\hline 28,439 & M & - & $\mathrm{CH}_{2} \mathrm{CF}_{2}$ \\
\hline 28,458 & M & - & $\mathrm{CH}_{2} \mathrm{CF}_{2}$ \\
\hline 28,495 & & - & $\mathrm{C}^{12} \mathrm{D}_{4} \mathrm{O}^{16}$ \\
\hline $28,518.14$ & W & $1-2, K=0$ & $\mathrm{C}^{13} \mathrm{H}_{3} \mathrm{I}$ \\
\hline $28,550.86$ & W & $1-2, K=0$ & $\mathrm{C}^{\cdot 3} \mathrm{H}_{3} \mathrm{I}$ \\
\hline 28,551 & W & - & $\mathrm{CH}_{2} \mathrm{CF}_{2}$ \\
\hline 28,575 & W & $-1-1-1,-$ & $\mathrm{CH}_{2} \mathrm{CF}_{2}$ \\
\hline $28,594.74$ & W & $1-2, K=1$ & $\mathrm{C}^{13} \mathrm{H}_{3} \mathrm{I}$ \\
\hline $28,604.73$ & $\mathrm{~S}$ & - & $\mathrm{N}^{14} \mathrm{H}_{3}$ \\
\hline 28,615 & W & - - - & $\mathrm{CH}_{2} \mathrm{CF}_{2}$ \\
\hline $28,650.91$ & $\mathrm{~W}$ & $1-2, K=0$ & $\mathrm{C}^{13} \mathrm{H}_{3} \mathrm{I}$ \\
\hline $28,687.21$ & W & $1-2, K=1$ & $\mathrm{C}^{13} \mathrm{H}_{3} \mathrm{I}$ \\
\hline 28,689 & W & 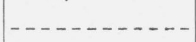 & $\mathrm{CH}_{2} \mathrm{CF}_{2}$ \\
\hline 28,852 & $\ldots$ & - & $\mathrm{CH}_{2} \mathrm{CF}_{2}$ \\
\hline 28,858 & $\ldots$ & -...-1 & $\mathrm{CH}_{2} \mathrm{CF}_{2}$ \\
\hline 28,954 & $\mathrm{~S}$ & - & $\mathrm{CH}_{2} \mathrm{CF}_{2}$ \\
\hline $28,974.85$ & & $\ldots-1$ & $\mathrm{H}_{2} \mathrm{CO}$ \\
\hline
\end{tabular}

TABLE 2. Lines listed by frequency-Continued

\begin{tabular}{|c|c|c|c|}
\hline Frequency & $\begin{array}{l}\text { Inten- } \\
\text { sity }\end{array}$ & Transition & Molecule \\
\hline $29,061.14$ & M & & $\mathrm{N}^{14} \mathrm{H}_{3}$ \\
\hline 29,080 & & $\ldots \ldots$ & $\mathrm{C}^{12} \mathrm{D}_{4} \mathrm{O}^{16}$ \\
\hline 29,460 & $\ldots$ & - - - & $\mathrm{SO}_{2}$ \\
\hline $29,598.95$ & M & $1-2, K=0$ & $\mathrm{C}^{12} \mathrm{H}_{3} \mathrm{I}$ \\
\hline $29,673.95$ & M & $1-2, K=0$ & $\mathrm{C}^{12} \mathrm{H}_{3} \mathrm{I}$ \\
\hline 29,688 & $\mathrm{~S}$ & - & $\mathrm{C}_{2}{ }^{12} \mathrm{H}_{4} \mathrm{O}^{16}$ \\
\hline 29,700 & & $5-6$ & $\mathrm{C}^{12} \mathrm{H}_{3} \mathrm{~N}^{14} \mathrm{C}^{12} \mathrm{~S}^{32}$ \\
\hline $29,735.71$ & M & $1-2, K=1$ & $\mathrm{C}^{12} \mathrm{H}_{3} \mathrm{I}$ \\
\hline $29,773.95$ & W & $1-2, K=0$ & $\mathrm{C}^{12} \mathrm{H}_{3} \mathrm{I}$ \\
\hline $29,782.71$ & M & $1-2, K=1$ & $\mathrm{C}^{12} \mathrm{H}_{3} \mathrm{I}$ \\
\hline $29,872.52$ & M & $1-2, K=0$ & $\mathrm{C}^{12} \mathrm{H}_{3} \mathrm{I}$ \\
\hline $29,914.66$ & $\mathrm{~S}$ & $\ldots \ldots-\ldots$ & $\mathrm{N}^{14} \mathrm{H}_{3}$ \\
\hline $29,923.50$ & M & $1-2, K=1$ & $\mathrm{C}^{12} \mathrm{H}_{3} \mathrm{I}$ \\
\hline $29,939.87$ & M & $1-2, K=1$ & $\mathrm{C}^{12} \mathrm{H}_{3} \mathrm{I}$ \\
\hline $29,986.84$ & W & $1-2, K=1$ & $\mathrm{C}^{12} \mathrm{H}_{3} \mathrm{I}$ \\
\hline $30,046.99$ & M & $1-2, K=0$ & $\mathrm{C}^{12} \mathrm{H}_{3} \mathrm{I}$ \\
\hline $30,075.08$ & M & $1-2, K=1$ & $\mathrm{C}^{12} \mathrm{H}_{3} \mathrm{I}$ \\
\hline $30,079.72$ & M & $1-2, K=0$ & $\mathrm{C}^{12} \mathrm{H}_{3} \mathrm{I}$ \\
\hline $30,121.32$ & M & $1-2, K=0$ & $\mathrm{C}^{12} \mathrm{H}_{3} \mathrm{I}$ \\
\hline $30,123.64$ & M & $1-2, K=1$ & $\mathrm{C}^{12} \mathrm{H}_{3} \mathrm{I}$ \\
\hline $30,179.71$ & M & $1-2, K=0$ & $\mathrm{C}^{12} \mathrm{H}_{3} \mathrm{I}$ \\
\hline $30,215.95$ & M & $1-2, K=1$ & $\mathrm{C}^{\prime 2} \mathrm{H}_{3} \mathrm{I}$ \\
\hline $30,453.46$ & M & $1-2, K=0$ & $\mathrm{C}^{12} \mathrm{H}_{3} \mathrm{I}$ \\
\hline $31,020.7$ & & $2-3$ & $\mathrm{CF}_{3} \mathrm{CH}_{3}$ \\
\hline $31,066.8$ & - - & $2-3$ & $\mathrm{CF}_{3} \mathrm{CH}_{3}$ \\
\hline $31,114.4$ & -- & $2-3$ & $\mathrm{CF}_{3} \mathrm{CH}_{3}$ \\
\hline $31,279.60$ & M & $1-2$ & $\mathrm{PF}_{3}$ \\
\hline $31,424.97$ & $\mathrm{~S}$ & - & $\mathrm{N}^{14} \mathrm{H}_{3}$ \\
\hline $31,718.28$ & M & $4-5, v=0$ & $\mathrm{IC}^{13} \mathrm{~N}^{14}$ \\
\hline $31,730.50$ & M & $4-5, v=0$ & $\mathrm{IC}^{13} \mathrm{~N}^{14}$ \\
\hline $31,741.50$ & W & $4-5, v=0$ & $\mathrm{IC}^{13} \mathrm{~N}^{14}$ \\
\hline $31,763.34$ & M & $4-5, v=0$ & $\mathrm{IC}^{13} \mathrm{~N}^{14}$ \\
\hline $31,783.31$ & M & $4-5, v=0$ & $\mathrm{IC}^{13} \mathrm{~N}^{14}$ \\
\hline $31,793.46$ & M & $4-5, v=0$ & $\mathrm{IC}^{13} \mathrm{~N}^{14}$ \\
\hline $31,848.77$ & M & $4-5, v=0$ & $\mathrm{IC}^{13} \mathrm{~N}^{14}$ \\
\hline 31,943 & -- & 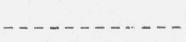 & $\mathrm{C}^{12} \mathrm{D}_{4} \mathrm{O}^{16}$ \\
\hline $32,200.58$ & $\mathrm{~S}$ & $4-5, v=0$ & $\mathrm{IC}^{12} \mathrm{~N}^{14}$ \\
\hline $32,203.57$ & $\mathrm{~S}$ & $4-5, v=0$ & $\mathrm{IC}^{12} \mathrm{~N}^{14}$ \\
\hline $32,215.56$ & $\mathrm{~S}$ & $4-5, v=0$ & $\mathrm{IC}^{12} \mathrm{~N}^{14}$ \\
\hline $32,226.85$ & $\mathrm{~S}$ & $4-5, v=0$ & $\mathrm{IC}^{12} \mathrm{~N}^{14}$ \\
\hline $32,248.52$ & $\mathrm{~S}$ & $4-5, v=0$ & $\mathrm{IC}^{12} \mathrm{~N}^{14}$ \\
\hline $32,268.33$ & $\mathrm{~S}$ & $4-5, v=0$ & $\mathrm{IC}^{12} \mathrm{~N}^{14}$ \\
\hline $32,278.55$ & $\mathrm{~S}$ & $4-5, v=0$ & $\mathrm{IC}^{12} \mathrm{~N}^{14}$ \\
\hline $32,386.29$ & $\mathrm{~S}$ & $4-5, v=0$ & $\mathrm{IC}^{12} \mathrm{~N}^{14}$ \\
\hline $32,392.59$ & M & $3-4, v=0$ & $\mathrm{Br}^{81} \mathrm{C}^{13} \mathrm{~N}^{14}$ \\
\hline $32,409.06$ & M & $3-4, v=0$ & $\mathrm{Br}^{81} \mathrm{C}^{13} \mathrm{~N}^{14}$ \\
\hline $32,581.73$ & M & $3-4, v=0$ & $\mathrm{Br}^{79} \mathrm{C}^{13} \mathrm{~N}^{14}$ \\
\hline
\end{tabular}


TABLE 2. Lines listed by frequency-Continued

\begin{tabular}{|c|c|c|c|}
\hline Frequency & $\begin{array}{c}\text { Inten- } \\
\text { sity }\end{array}$ & Transition & Molecule \\
\hline $32,601.46$ & M & $3-4, v=0$ & $\mathrm{Br}^{79} \mathrm{C}^{13} \mathrm{~N}^{14}$ \\
\hline $32,643.13$ & M & $3-4, v=0$ & $\mathrm{Br}^{81} \mathrm{C}^{12} \mathrm{~N}^{14}$ \\
\hline $32,720.28$ & M & $3-4, v=0$ & $\mathrm{Br}^{81} \mathrm{C}^{12} \mathrm{~N}^{14}$ \\
\hline $32,770.13$ & $\mathrm{~S}$ & $3-4, v=0$ & $\mathrm{Br}^{81} \mathrm{C}^{12} \mathrm{~N}^{14}$ \\
\hline $32,786.65$ & M & $3-4, v=0$ & $\mathrm{Br}^{81} \mathrm{C}^{12} \mathrm{~N}^{14}$ \\
\hline $32,804.56$ & M & $3-4, v=0$ & $\mathrm{Br}^{79} \mathrm{C}^{12} \mathrm{~N}^{14}$ \\
\hline $32,913.24$ & M & $3-4, v=0$ & $\mathrm{Br}^{81} \mathrm{C}^{12} \mathrm{~N}^{14}$ \\
\hline $32,956.68$ & M & $3-4, v=0$ & $\mathrm{Br}^{79} \mathrm{C}^{12} \mathrm{~N}^{14}$ \\
\hline $32,976.40$ & $\mathrm{~S}$ & $3-4, v=0$ & $\mathrm{Br}^{79} \mathrm{C}^{12} \mathrm{~N}^{14}$ \\
\hline $33,156.95$ & $\mathrm{~S}$ & & $\mathrm{~N}^{14} \mathrm{H}_{3}$ \\
\hline 33,285 & - & & $\mathrm{C}^{12} \mathrm{D}_{4} \mathrm{O}^{16}$ \\
\hline 34,150 & & $-1 . .-1$ & $\mathrm{C}^{12} \mathrm{H}_{4} \mathrm{O}^{16}$ \\
\hline 34,158 & $\ldots$ & - - - & $\mathrm{C}^{12} \mathrm{H}_{4} \mathrm{O}^{16}$ \\
\hline $34,627.24$ & M & $1-2, K=1$ & $\mathrm{~B}^{11} \mathrm{H}_{3} \mathrm{C}^{12} \mathrm{O}^{16}$ \\
\hline $34,627.50$ & M & $1-2, K=1$ & $\mathrm{~B}^{11} \mathrm{H}_{3} \mathrm{C}^{12} \mathrm{O}^{16}$ \\
\hline $34,627.73$ & M & $1-2, K=1$ & $\mathrm{~B}^{11} \mathrm{H}_{3} \mathrm{C}^{12} \mathrm{O}^{16}$ \\
\hline $34,627.89$ & M & $1-2, K=1$ & $\mathrm{~B}^{11} \mathrm{H}_{3} \mathrm{C}^{12} \mathrm{O}^{16}$ \\
\hline $34,628.62$ & M & $1-2, K=0$ & $\mathrm{~B}^{11} \mathrm{H}_{3} \mathrm{C}^{12} \mathrm{O}^{16}$ \\
\hline $34,628.90$ & $\mathrm{~S}$ & $1-2, K=0$ & $\mathrm{~B}^{11} \mathrm{H}_{3} \mathrm{C}^{12} \mathrm{O}^{16}$ \\
\hline $34,629.32$ & M & $1-2, K=0$ & $\mathrm{~B}^{11} \mathrm{H}_{3} \mathrm{C}^{12} \mathrm{O}^{16}$ \\
\hline $34,889.05$ & W & $2-3, v=0$ & $\mathrm{Cl}^{37} \mathrm{C}^{13} \mathrm{~N}^{14}$ \\
\hline $35,067.99$ & M & $2-3, v=0$ & $\mathrm{C}^{37} \mathrm{C}^{12} \mathrm{~N}^{14}$ \\
\hline 35,068 & - & - & $\mathrm{C}^{12} \mathrm{D}_{4} \mathrm{O}^{16}$ \\
\hline $35,080.39$ & $\mathrm{~S}$ & $2-3, v=0$ & $\mathrm{Cl}^{37} \mathrm{C}^{12} \mathrm{~N}^{14}$ \\
\hline $35,084.15$ & $\mathrm{~S}$ & $\therefore-3, v=0$ & $\mathrm{Cl}^{37} \mathrm{C}^{12} \mathrm{~N}^{14}$ \\
\hline $35,091.97$ & $\mathrm{M}$ & $2-3, v=0$ & $\mathrm{Cl}^{37} \mathrm{C}^{12} \mathrm{~N}^{14}$ \\
\hline $35,134.44$ & M & $2-3, v=0$ & $\mathrm{~N}^{14} \mathrm{H}_{3}$ \\
\hline 35,341 & $\ldots$ & - - & $\mathrm{C}^{12} \mathrm{D}_{4} \mathrm{O}^{16}$ \\
\hline $35,618.81$ & W & $2-3, v=0$ & $\mathrm{Cl}^{35} \mathrm{C}^{3} \mathrm{~N}^{14}$ \\
\hline $35,634.85$ & M & $2-3, v=0$ & $\mathrm{C}]^{35} \mathrm{C}^{13} \mathrm{~N}^{14}$ \\
\hline $35,639.78$ & M & $2-3, v=0$ & $\mathrm{Cl}^{35} \mathrm{C}^{13} \mathrm{~N}^{14}$ \\
\hline $35,649.56$ & W & $2-3, v=0$ & $\mathrm{Cl}^{35} \mathrm{C}^{13} \mathrm{~N}^{14}$ \\
\hline $35,805.09$ & $\mathrm{~S}$ & $2-3, v=0$ & $\mathrm{Cl}^{35} \mathrm{C}^{12} \mathrm{~N}^{14}$ \\
\hline $35,820.65$ & $\mathrm{~S}$ & $2-3, v=0$ & $\mathrm{Cl}^{35} \mathrm{C}^{12} \mathrm{~N}^{14}$ \\
\hline $35,825.95$ & $\mathrm{~S}$ & $2-3, v=0$ & $\mathrm{Cl}^{35} \mathrm{C}^{12} \mathrm{~N}^{14}$ \\
\hline $35,835.74$ & $\mathrm{~S}$ & $2-3, v=0$ & $\mathrm{Cl}^{35} \mathrm{C}^{12} \mathrm{~N}^{14}$ \\
\hline $35,917.62$ & M & $1-2, K=1$ & $\mathrm{~B}^{10} \mathrm{H}_{3} \mathrm{C}^{12} \mathrm{O}^{16}$ \\
\hline $35,917.88$ & M & $1-2, K=1$ & $\mathrm{~B}^{10} \mathrm{H}_{3} \mathrm{C}^{12} \mathrm{O}^{16}$ \\
\hline $35,918.20$ & W & $1-2, K=1$ & $\mathrm{~B}^{10} \mathrm{H}_{3} \mathrm{C}^{12} \mathrm{O}^{16}$ \\
\hline $35,918.50$ & M & $1-2, K=1$ & $\mathrm{~B}^{10} \mathrm{H}_{3} \mathrm{C}^{12} \mathrm{O}^{16}$ \\
\hline $35,919.02$ & W & $1-2, K=0$ & $\mathrm{~B}^{10} \mathrm{H}_{3} \mathrm{C}^{12} \mathrm{O}^{16}$ \\
\hline $35,919.53$ & M & $1-2, K=0$ & $\mathrm{~B}^{10} \mathrm{H}_{3} \mathrm{C}^{12} \mathrm{O}^{16}$ \\
\hline $35,919.86$ & M & $1-2, K=0$ & $\mathrm{~B}^{10} \mathrm{H}_{3} \mathrm{C}^{12} \mathrm{O}^{16}$ \\
\hline $35,920.14$ & M & $1-2, K=0$ & $\mathrm{~B}^{10} \mathrm{H}_{3} \mathrm{C}^{12} \mathrm{O}^{16}$ \\
\hline
\end{tabular}

TABLE 2. Lines listed by frequency-Continued

\begin{tabular}{|c|c|c|c|}
\hline Frequency & $\begin{array}{l}\text { Inten- } \\
\text { sity }\end{array}$ & Transition & Molecule \\
\hline $36,488.82$ & $\mathrm{~S}$ & $2-3, v=0$ & $\mathrm{O}^{16} \mathrm{C}^{12} \mathrm{~S}^{32}$ \\
\hline 36,632 & M & - - - & $\mathrm{CH}_{2} \mathrm{CF}_{2}$ \\
\hline 36,791 & $\mathrm{~S}$ & & $\mathrm{C}_{2}{ }^{12} \mathrm{H}_{4} \mathrm{O}^{16}$ \\
\hline $36,793.64$ & & $0-1$ & $\mathrm{C}^{12} \mathrm{H}_{3} \mathrm{C}^{12} \mathrm{~N}^{14}$ \\
\hline $36,794.26$ & & $0-1$ & $\mathrm{C}^{12} \mathrm{H}_{3} \mathrm{C}^{12} \mathrm{~N}^{14}$ \\
\hline $36,794.88$ & & $0-1$ & $\mathrm{C}^{12} \mathrm{H}_{3} \mathrm{C}^{12} \mathrm{~N}^{14}$ \\
\hline $36,795.38$ & 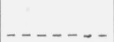 & $0-1$ & $\mathrm{C}^{12} \mathrm{H}_{3} \mathrm{C}^{12} \mathrm{~N}^{14}$ \\
\hline $36,796.27$ & & $0-1$ & $\mathrm{C}^{12} \mathrm{H}_{3} \mathrm{C}^{12} \mathrm{~N}^{14}$ \\
\hline $36,797.52$ & $\ldots$ & $0-1$ & $\mathrm{C}^{12} \mathrm{H}_{3} \mathrm{C}^{12} \mathrm{~N}^{14}$ \\
\hline $36,870.94$ & - & $0-1$ & $\mathrm{C}^{12} \mathrm{H}_{3} \mathrm{C}^{12} \mathrm{~N}^{14}$ \\
\hline $36,903.40$ & - & $0-1$ & $\mathrm{C}^{12} \mathrm{H}_{3} \mathrm{C}^{12} \mathrm{~N}^{14}$ \\
\hline $36,942.15$ & & $0-1$ & $\mathrm{C}^{12} \mathrm{H}_{3} \mathrm{C}^{12} \mathrm{~N}^{14}$ \\
\hline 37,329 & $\mathrm{~S}$ & & $\mathrm{C}_{2}{ }^{12} \mathrm{H}_{4} \mathrm{O}^{16}$ \\
\hline $37,385.18$ & M & $\ldots$ & $\mathrm{N}^{14} \mathrm{H}_{3}$ \\
\hline 37,781 & $\mathrm{~S}$ & - & $\mathrm{C}_{2}{ }^{12} \mathrm{H}_{4} \mathrm{O}^{16}$ \\
\hline $38,006.47$ & W & $1-2, K=1$ & $\mathrm{CH}_{3} \mathrm{Br}^{8 \mathrm{i}}$ \\
\hline $38,030.77$ & M & $1-2, K=0$ & $\mathrm{CH}_{3} \mathrm{Br}^{81}$ \\
\hline $38,066.72$ & W & $1-2, K=1$ & $\mathrm{CH}_{3} \mathrm{Br}^{81}$ \\
\hline $38,097.45$ & M & $1-2, K=1$ & $\mathrm{CH}_{3} \mathrm{Br}^{81}$ \\
\hline $38,116.65$ & M & $1-2, K=0$ & $\mathrm{CH}_{3} \mathrm{Br}^{81}$ \\
\hline $38,126.97$ & M & $1-2, K=0$ & $\mathrm{CH}_{3} \mathrm{Br}^{81}$ \\
\hline $38,128.40$ & W & $1-2, K=1$ & $\mathrm{C}^{12} \mathrm{H}_{3} \mathrm{Br}^{79}$ \\
\hline $38,157.30$ & M & $1-2, K=0$ & $\mathrm{C}^{12} \mathrm{H}_{3} \mathrm{Br}^{79}$ \\
\hline $38,157.70$ & W & $1-2, K=1$ & $\mathrm{C}^{12} \mathrm{H}_{3} \mathrm{Br}^{81}$ \\
\hline $38,175.08$ & M & $1-2, K=1$ & $\mathrm{C}^{12} \mathrm{H}_{3} \mathrm{Br}^{81}$ \\
\hline $38,200.52$ & W & $1-2, K=1$ & $\mathrm{C}^{12} \mathrm{H}_{3} \mathrm{Br}^{79}$ \\
\hline $38,218.21$ & M & $1-2, K=1$ & $\mathrm{C}^{12} \mathrm{H}_{3} \mathrm{Br}^{81}$ \\
\hline $38,237.14$ & M & $1-2, K=1$ & $\mathrm{C}^{12} \mathrm{H}_{3} \mathrm{Br}^{79}$ \\
\hline $38,247.77$ & M & $1-2, K=0$ & $\mathrm{C}^{12} \mathrm{H}_{3} \mathrm{Br}^{81}$ \\
\hline $38,260.10$ & M & $1-2, K=0$ & $\mathrm{C}^{12} \mathrm{H}_{3} \mathrm{Br}^{79}$ \\
\hline $38,272.40$ & M & $1-2, K=0$ & $\mathrm{C}^{12} \mathrm{H}_{3} \mathrm{Br}^{79}$ \\
\hline $38,309.45$ & W & $1-2, K=1$ & $\mathrm{C}^{12} \mathrm{H}_{3} \mathrm{Br}^{79}$ \\
\hline $38,330.25$ & M & $1-2, K=1$ & $\mathrm{C}^{12} \mathrm{H}_{3} \mathrm{Br}^{79}$ \\
\hline $38,381.70$ & M & $1-2, K=1$ & $\mathrm{C}^{12} \mathrm{H}_{3} \mathrm{Br}^{79}$ \\
\hline $38,404.49$ & M & $1-2, K=0$ & $\mathrm{C}^{12} \mathrm{H}_{3} \mathrm{Br}^{79}$ \\
\hline $38,417.09$ & M & $1-2, K=0$ & $\mathrm{C}^{12} \mathrm{H}_{3} \mathrm{Br}^{79}$ \\
\hline 38,702 & $\mathrm{~S}$ & - - & $\mathrm{C}_{2}{ }^{12} \mathrm{H}_{4} \mathrm{O}^{16}$ \\
\hline $38,782.21$ & & $1-2, K=1$ & $\mathrm{C}^{12} \mathrm{H}_{3} \mathrm{~N}^{14} \mathrm{C}^{13}$ \\
\hline $38,783.21$ & $\cdots-$ & $1-2, K=0$ & $\mathrm{C}^{12} \mathrm{H}_{3} \mathrm{~N}^{14} \mathrm{C}^{13}$ \\
\hline 39,582 & M & - & $\mathrm{C}_{2}{ }^{12} \mathrm{H}_{4} \mathrm{O}^{16}$ \\
\hline 39,592 & & & $\mathrm{C}^{12} \mathrm{D}_{4} \mathrm{O}^{16}$ \\
\hline 39,677 & $\mathrm{~S}$ & - & $\mathrm{C}_{2}{ }^{12} \mathrm{H}_{4} \mathrm{O}^{16}$ \\
\hline $39,941.54$ & M & - - - - - & $\mathrm{N}^{14} \mathrm{H}_{3}$ \\
\hline $40,210.27$ & & $1-2, K=1$ & $\mathrm{C}^{12} \mathrm{H}_{3} \mathrm{~N}^{14} \mathrm{C}^{12}$ \\
\hline
\end{tabular}


TABle 2. Lines listed by frequency-Continued

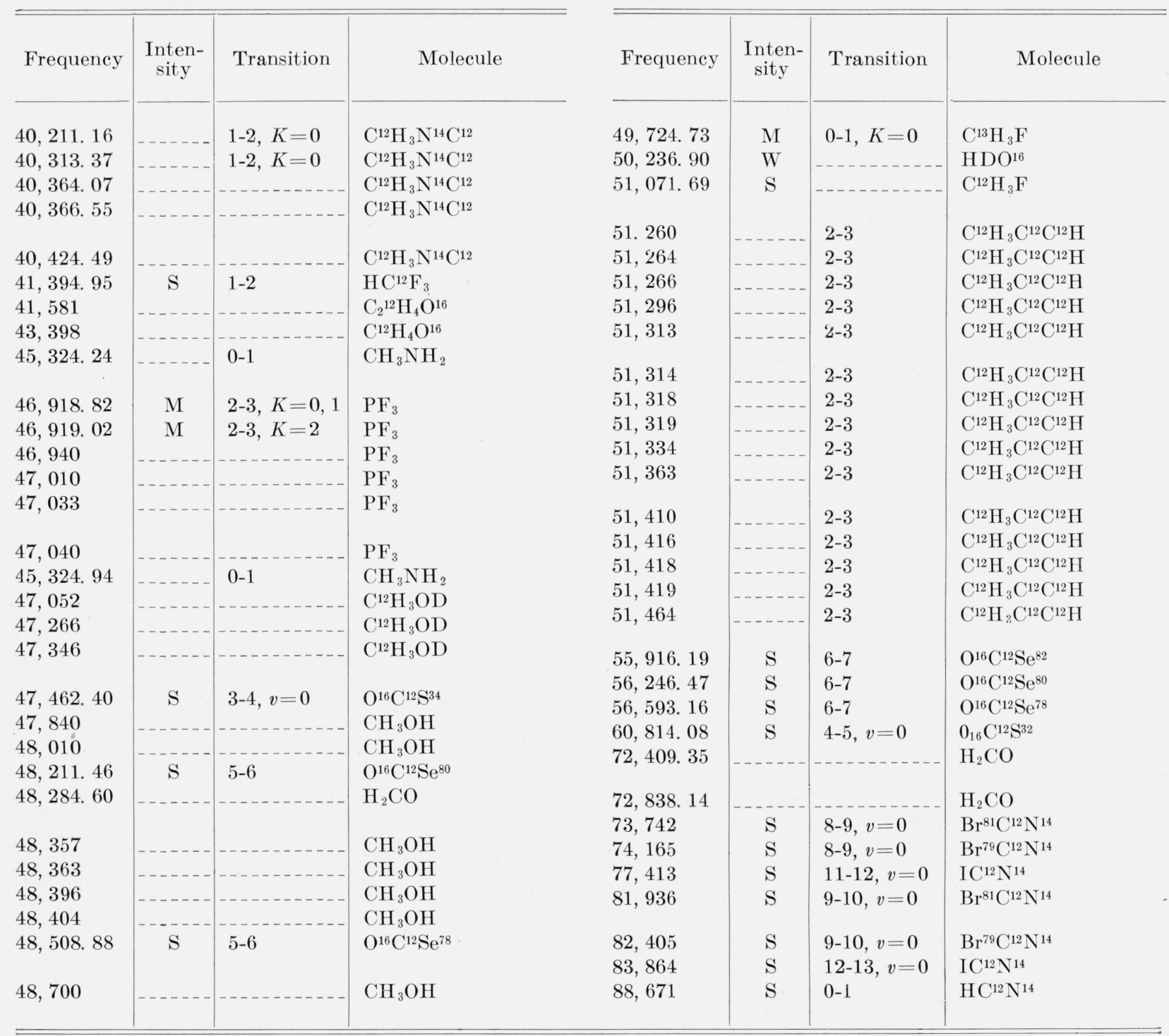

TaBle 2. Lines listed by frequency-Continued 
TABLE 3. Values of $\left[3{ }^{3} C(C+1)-I(I+1) J(J+1)\right] / 2 I(2 I-1)(2 J-1)(2 J+3)$, where $C=F(F+1)-I(I+1)-J(J+1)$

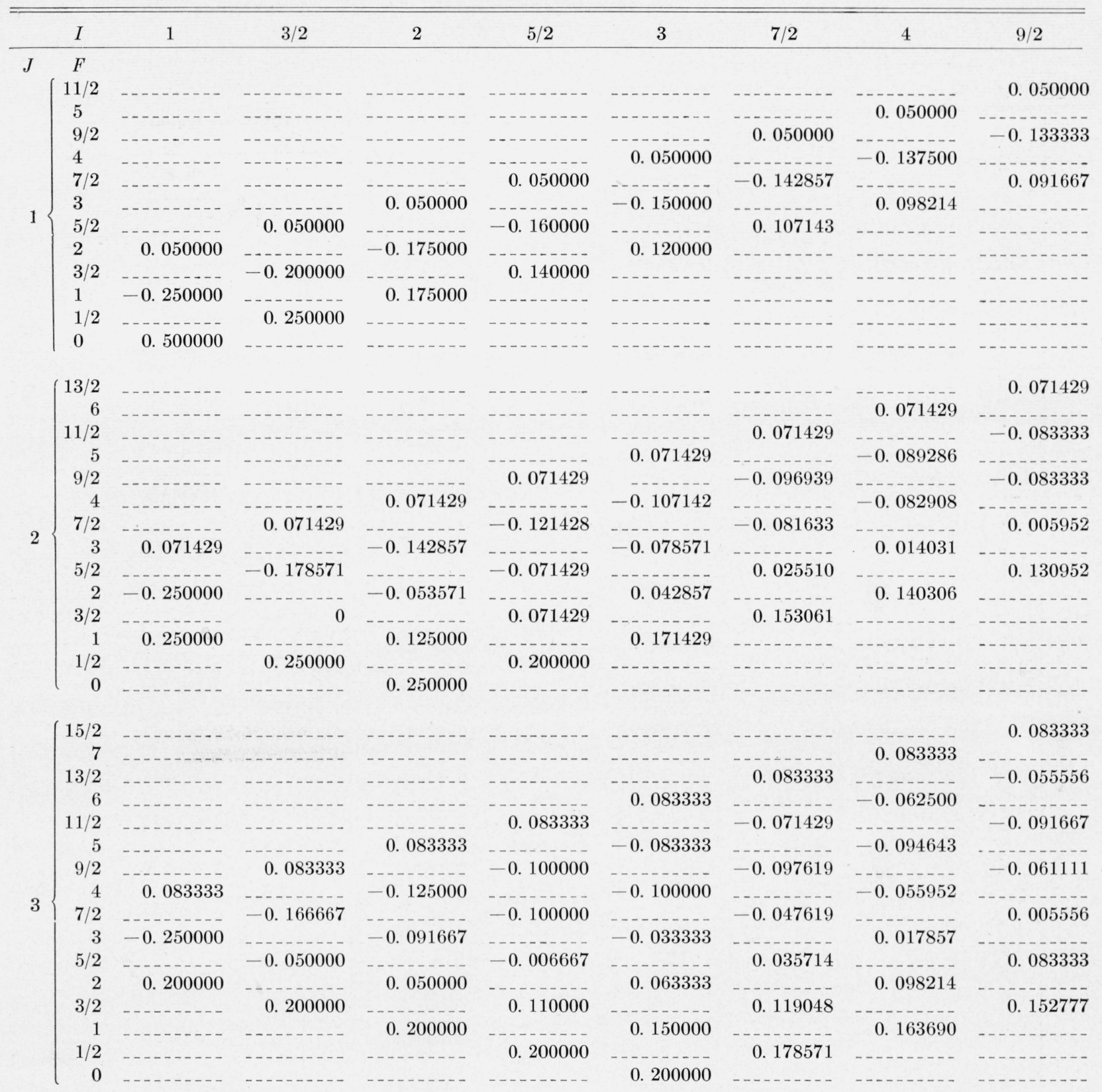


TABLE 3. Values of $[3 / 4 C(C+1)-I(I+1) J(J+1)] / 2 I(2 I-1)(2 J-1)(2 J+3)$, where $C=F(F+1)-I(I+1)-J(J+1)-$ Continued

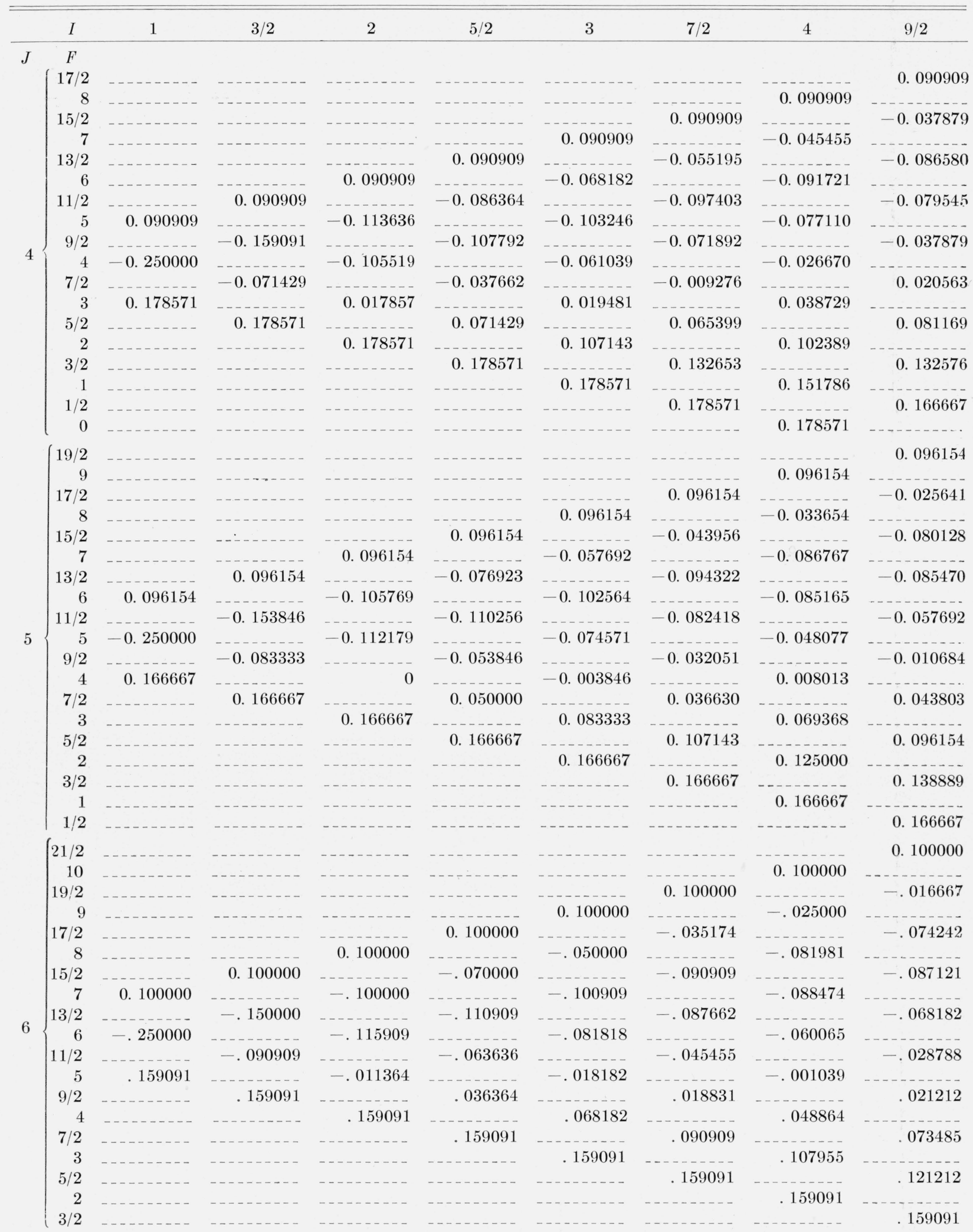


TABLE 3. Values of $[3 / 4 C(C+1)-I(I+1) J(J+1)] / 2 I(2 I-1)(2 J-1)(2 J+3)$, where $C=F(F+1)-I(I+1)-J(J+1)-$ Continued

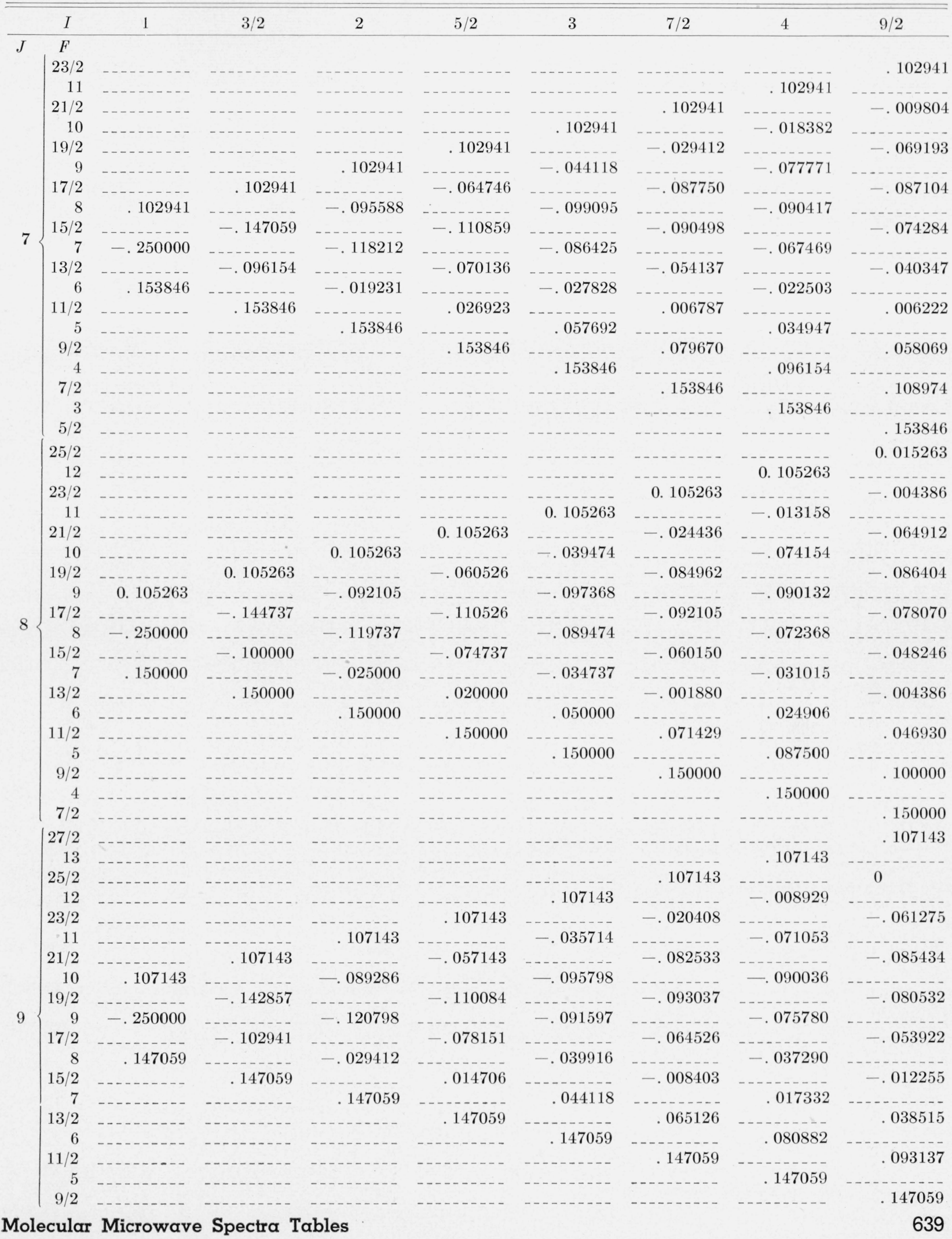


TABLE 3. Values of $[3 / 4 C(C+1)-I(I+1) J(J+1)] / 2 I(2 I-1)(2 J-1)(2 J+3)$, where $C=F(F+1)-I(I+1)-J(J+1)-$ Continued

\begin{tabular}{|c|c|c|c|c|c|c|c|c|c|}
\hline & $I$ & 1 & $3 / 2$ & 2 & $5 / 2$ & 3 & $7 / 2$ & 4 & $9 / 2$ \\
\hline \multirow{7}{*}{$J$} & $29 / 2$ & - n - & - - & 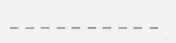 & -1 & & & & 0. 108696 \\
\hline & $27 / 2$ & $-\ldots-\ldots$ & - - & $-\ldots-\ldots--$ & 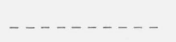 & $-\ldots-1--$ & 0.018696 & - - - - & -.003623 \\
\hline & 13 & - - - - - - & - - & - - - - - - & 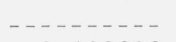 & 0. 108696 & $-\ldots \ldots-\ldots-$ & -.005435 & - \\
\hline & $25 / 2$ & $\ldots-\ldots-\ldots$ & - - - - & $\ldots \ldots$ & 0. 108696 & $\ldots$ & -.017081 & - & -.058162 \\
\hline & 11 & 0. 108696 & - & -.086957 & - - - - - & -.094394 & $-\ldots--1--$ & -.089715 & - \\
\hline & $21 / 2$ & - - - - - - - & -. 141304 & - - - - - - - & -.109610 & - - - - - & -.093576 & - - - - - - - & -.082189 \\
\hline & 10 & -.250000 & - - - & -.121568 & - & -.093135 & - & -.078253 & $\ldots$ \\
\hline \multirow[t]{5}{*}{10} & $19 / 2$ & - & -.105263 & $\ldots \ldots \ldots$ & -.080778 & - - - - - - - & -.067833 & - - - - - & -.058162 \\
\hline & 9 & .144737 & $-\ldots-\ldots-\ldots$ & -.032895 & - - - & -.043936 & - - - - - & -.042089 & $-\ldots$ \\
\hline & $13 / 2$ & $\ldots \ldots$ & - & $\ldots \ldots \ldots$ & $-\ldots-\ldots-\ldots$ & - & .144737 & - - - & .087719 \\
\hline & 6 & - - - & - - & - & - - - & - - - & - - - - - - - & .144737 & $\ldots$ \\
\hline & $11 / 2$ & - - - & - & $\ldots \ldots$ & - - & $-\ldots--$ & $-\ldots-\ldots$ & $-\ldots----$ & .144737 \\
\hline
\end{tabular}

\section{References}

[1] C. I. Beard and B. P. Dailey, J. Chem. Phys. 15, 762 (1947).

[1a] C. I. Beard and B. P. Dailey, MITRLE Tech. Report \#79 (1948).

[2] G. E. Becker and S. H. Autler, Phys. Rev. 70, 300 (1946).

[3] R. Beringer, Phys. Rev. 70, 53 (1946).

[4] B. Bleaney and R. P. Penrose, Proc. Roy. Soc. A189, 358 (1947).

[4a] B. Bleaney and R. P. Penrose, Proc. Phys. Soc. (London) 60, 540 (1947).

[4b] B. Bleaney and J. H. N. Loubser, Nature 161, 522 (1948).

[4c] A. C. Candler, Atomic spectra and the vector model (Cambridge Univ. Press, Cambridge, Mass., 1937).

[5] H. B. G. Casimir, On the interaction between atomic nuclei and electrons (DeErven F. Bohn, Haarlem, 1936).

[6] D. K. Coles, Phys. Rev. 74, 1194 (1948).

[7] D. K. Coles, E. S. Elyash, and J. G. Gorman, Phys. Rev. $\boldsymbol{7}, 973$ (1947).

[7a] D. K. Coles and W. E. Good, Phys. Rev. 70, 979 (1946).

[7b] D. K. Coles and R. H. Hughes, private communication.

[8] E. U. Condon and G. H. Shortley, The theory of atomic spectra (Macmillan and Co., New York, N. Y., 1935).

[9] P. C. Cross, R. M. Hainer, and G. W. King, J. Chem. Phys. 12, 210 (1944).

[10] G. L. Cunningham, W. I. LeVan, and W. D. Gwinn, Phys. Rev. 74, 1537 (1948).

[10a] G. L. Cunningham, A. W. Boyd, W. D. Gwinn, and W. I. LeVan, private commurication.
[11] B. P. Dailey, private communication.

[12] B. P. Dailey, S. Golden, and E. B. Wilson, Jr., Phys. Rev. 79, 871 (1947).

[12a] B. P. Dailey, R. L. Kyhl, M. W. P. Strandberg, J. H. VanVleck, and E. B. Wilson, Jr., Phys. Rev. 70, 984 (1946).

[13] B. P. Dailey, K. Rusinow, R. G. Shulman, and C. H. Townes, Phys. Rev. 84, 1245 (1948); also private communication.

[14] B. P. Dailey and E. B. Wilson, Jr., Phys. Rev. 7\%, 84 (1947).

[15] B. P. Dailey and E. B. Wilson, Jr., Phys. Rev. 72, 522 (1947).

[16] T. W. Dakin, W. E. Good, and D. K. Coles, Phys. Rev. \%1, 640 (1947).

[17] W. F. Edgell and A. Roberts, J. Chem. Phys. 16, 1002 (1948).

[18] H. D. Edwards, O. R. Gilliam, and W. Gordy, to be published.

[19] O. R. Gilliam, H. D. Edwards, and W. Gordy, Phys. Rev. 75, 1014 (1949)

[20] S. Golden, T. Wentink, Jr., R. E. Hillger, and M. W. P. Strandberg, Phys. Rev. 73, 92 (1948).

[20a] J. H. Goldstein and J. K. Bragg, Phys. Rev. 75, 1453 (1949).

[21] W. E. Good and D. K. Coles, Phys, Rev. 71, 383 (1947).

[22] W. Gordy, H. Ring, and A. B. Burg, Phys. Rev. 74, 1191 (1948).

[23] W. Gordy, J. W. Simmons, and A. G. Smith, Phys. Rev. 74, 243 (1948).

[23a] W. D. Gwinn, private communication.

[24] R. M. Hainer, G. W. King, and P. C. Cross, Phys. Rev. 70, 108 (1948).

[24a] R. S. Henderson, Phys. Rev. 74, 107 and $\mathbf{7 4 ,} 626$ (1948). 
[25] W. D. Hershberger and J. Turkevitch, Phys. Rev. $\mathbf{1 1}, 554$ (1947); and private communication.

[26] G. Herzberg, Molecular spectra and molecular structure; II infrared and Raman spectra of polyatomic molecules (D. VanNostrand \& Co., New York, N. Y., 1945).

[27] R. E. Hillger, M. W. P. Strandberg, T. Wentink, Jr., and R. L. Kyhl, Phys. Rev. 72, 157 (1947).

[27a] J. M. Jauch, Phys. Rev. 72, 715 (1947).

[27b] R. Karplus and A. H. Sharbaugh, Phys. Rev. 75, 889 (1949).

[28] M. Kessler, H. Ring, and W. Gordy, quoted by H. H. Nielsen. Phys. Rev. \%5, 1961 (1949) and private communication.

[29] G. W. King, R. M. Hainer, and P. C. Cross, J. Chem. Phys. 11, 27 (1943).

[30] J. Klein, W. Low, and C. H. Townes, private communication.

[31] H. R. L. Lamont, Phys. Rev. 74, 353 (1948).

[32] W. Low and C. H. Townes, Phys. Rev. 75, 529 (1949).

[33] C. H. Millar, thesis, MeGill University (1947).

[33a] M. Mizoshima, Phys. Rev. 74, 705 (1948).

[34] H. H. Nielsen and D. M. Dennison, Phys. Rev. 72, 1101 (1947).

[35] H. Ring, H. D. Edwards, W. Gordy, and O. R. Gilliam, to be published.

[36] H. Ring, H. D. Edwards, M. Kessler, and W. Gordy, Phys. Rev. 7\%, 1262 (1947).

[37] A. Roberts, Phys. Rev. 73, 1405 (1948).

[37a] A. Roberts and W. F. Edgell, J. Chem. Phys. 17, 742 (1949).

[37b] A. Roberts, private communication.

[38] A. H. Sharbaugh, Phys. Rev. 94, 1870 (1948); and private communication.

[38a] A. H. Sharbaugh and J. Mattern, Phys. Rev. 75, 1102 (1949).

[39] R. G. Shulman, B. P. Dailey, and C. H. Townes, Phys. Rev. 74, 846 (1948).

[39a] R. G. Shulman and H. Minden, private communication.

[39b] R. G. Shulman and C. H. Townes, private communication.

[39c] R. G. Shulman, private communication.

[40] J. W. Simmons and W. Gordy, Phys. Rev. 73, 713 (1947).

[41] A. G. Smith, W. Gordy, J. W. Simmons, and W. V. Smith, Phys. Rev. \%5, 260 (1949).

[42] A. G. Smith, H. Ring, W. V. Smith, and W. Gordy, Phys. Rev. 74, 370 (1948).
[43] A. G. Smith, H. Ring, W. V. Smith, and W. Gordy, Phys. Rev. 73, 635 (1948).

[44] M. W. P. Strandberg, paper delivered at Washington meeting of the American Physical Society (1948).

[45] M. W. P. Strandberg, R. L. Kyhl, R. E. Hillger, and T. Wentink, Jr., Phys. Rev. \%1, 326 (1947).

[46] M. W. P. Strandberg, R. L. Kyhl, R. E. Hillger, and T. Wentink, Jr., Phys. Rev. $\mathbf{7 1 ,} 639$ (1947).

[47] M. W. P. Strandberg, T. Wentink, Jr., and A. G. Hill, Phys. Rev. 75, 827 (1949).

[48] M. W. P. Strandberg, T. Wentink, Jr., R. E. Hillger, G. H. Wannier, and M. L. Deutsch, Phys. Rev. 73, 188 (1948).

[48a] M. W. P. Strandberg, C. Y. Meng, and J. G. Ingersoll, Phys. Rev. 75, 1524 (1949).

[49] M. W. P. Strandberg, T. Wentink, Jr., and R. L. Kyhl, MITRLE Rept. 59 (1948).

[49a] M. W. P. Strandberg, private communication.

[49b] M. W. P. Strandberg, MITRLE Report \#85 (1948).

[49e] M. W. P. Strandberg, et al., MITRLE Quarterly Progress Report (Oct. 15, 1948).

[49d] M. W. P. Strandberg, C. S. Pearsall, and M. T. Weiss, J. Chem. Phys. 17, 429 (1949).

[50] C. H. Townes, Phys. Rev. 70, 665 (1946).

[51] C. H. Townes and J. Bardeen, Phys. Rev. 73, 97 (1948).

[52] C. H. Townes and S. Geschwind, Phys. Rev. 74, 626 (1948).

[53] C. H. Townes, A. N. Holden, and F. R. Merritt, Phys. Rev. 74, 1113 (1948)

[54] C. H. Townes, A. N. Holden, and F. R. Merritt, Phys. Rev. 73, 1334 (1948).

[55] C. H. Townes and F. R. Merritt, Phys. Rev. 70, 558 (1946).

[57] T. E. Turner, thesis, McGill University (1948).

[58] J. H. Van Vleck and V. Weisskopf, Rev. Mod. Phys. 17, 227 (1945).

[58a] J. H. Van Vleck, Phys. Rev. 71, 468 (1947).

[58b] J. H. Van Vleck, Phys. Rev. \%1, 413 (1947).

[59] R. T. Weidner, Phys. Rev. 73, 254 and 72,1268 (1947-48).

[59a] I. R. Weingarten, The absorption of microwaves by gases at high pressure, thesis, Columbia University (1948).

[60] H. E. White, Introduction to atomic spectra (McGraw Hill Book Co., Inc., New York, N. Y., 1948).

[61] T. Y. Wu, Vibrational spectra and the structure of polyatomic molecules (J. W. Edwards, Ann Arbor, Mich., 1946). 


\section{Index}

By Chemical Symbol

1. $\mathrm{AsF}_{3}$

2. $\mathrm{BH}_{3} \mathrm{CO}$

3. $\mathrm{BrCN}$

4. $\mathrm{CF}_{2} \mathrm{CH}_{2}$

5. $\mathrm{CF}_{3} \mathrm{CH}_{3}$

6. $\mathrm{CHClF}_{2}$

7. $\mathrm{CH}_{2} \mathrm{Br}_{2}$

8. $\mathrm{CH}_{2} \mathrm{CF}_{2}$

9. $\mathrm{CH}_{2} \mathrm{Cl}_{2}$

10. $\mathrm{CH}_{3} \mathrm{Br}$

11. $\mathrm{CH}_{3} \mathrm{CCH}$

12. $\mathrm{CH}_{3} \mathrm{Cl}$

13. $\mathrm{CH}_{3} \mathrm{CN}$

14. $\mathrm{CH}_{3} \mathrm{~F}$

15. $\mathrm{CH}_{3} \mathrm{I}$

16. $\mathrm{CH}_{3} \mathrm{NC}$

17. $\mathrm{CH}_{3} \mathrm{NCS}$

18. $\mathrm{CH}_{3} \mathrm{NH}_{2}$

19. $\mathrm{CH}_{3} \mathrm{NO}_{2}$

20. $\mathrm{CH}_{3} \mathrm{OH}$

21. $\mathrm{CH}_{3} \mathrm{SCN}$

22. $\mathrm{C}_{2} \mathrm{H}_{3} \mathrm{Cl}$

23. $\mathrm{C}_{2} \mathrm{H}_{4} \mathrm{O}$

24. $\mathrm{C}_{3} \mathrm{H}_{4}$

25. $\mathrm{ClCN}$

26. COS

27. $\mathrm{COSe}$

28. $\mathrm{HCF}_{3}$

29. $\mathrm{HCN}$

30. $\mathrm{H}_{2} \mathrm{CO}$

31. $\mathrm{H}_{2} \mathrm{O}$

32. HNCS

33. ICl

34. ICN

35. $\mathrm{NH}_{3}$

36. $\mathrm{N}_{2} \mathrm{O}$

37. $\mathrm{O}_{2}$

38. OCS

39. OCSe

40. $\mathrm{PF}_{3}$

41. $\mathrm{SiH}_{3} \mathrm{Cl}$

42. $\mathrm{SO}_{2}$

43. $\mathrm{SPF}_{3}$
Page

614

614

615

616

616

616

616

616

617

617

618

618

618

618

618

619

619

619

620

620

620

620

621

618

621

626

627

622

622

622

622

622

623

623

624

626

626

626

627

627

627

628

628

Ammonia

By Name

Arsenic trifluoride _... 614

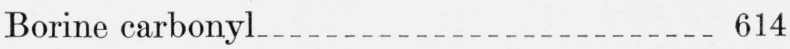

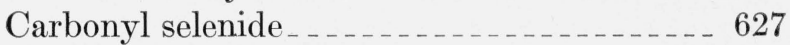

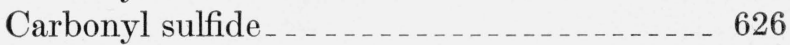

Chlorodifluoromethane _._._._. _._. _. 616

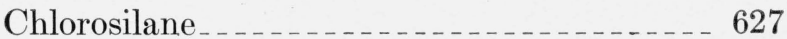

Cyanogen bromide _.............. 615

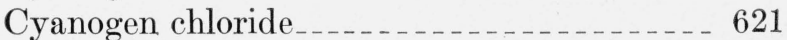

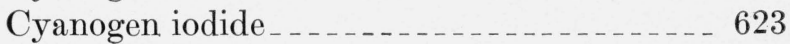

Difluorochloroform _._. _ _ _ 616

Difluoroethylene (Uns.) _._._._._._._._. 616

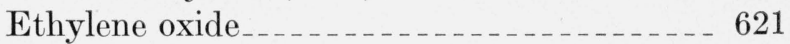

Fluoroform _...

Formaldehyde _...................... 622

Hydrocyanic acid _.................... 622

Iodine chloride_...

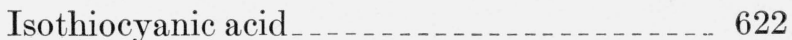

Methanol _.......................... 620

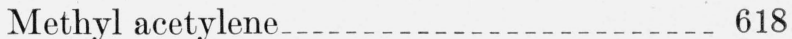

Methyl alcohol_._._._._. 620

Methyl bromide_____________ 617

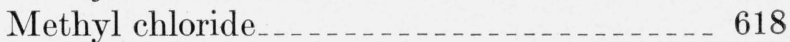

Methyl cyanide_._._._._._._._._._. 618

Methyl fluoride_._._._._._._._._._. 618

Methyl fluoroform _._._._. 616

Methyl iodide_........... 618

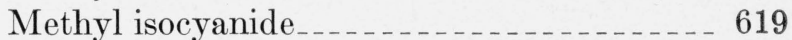

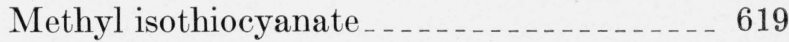

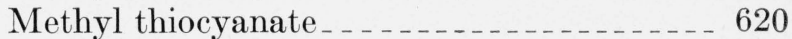

Methylamine _._._._._._._._._._._. 619

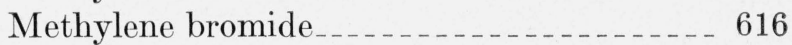

Methylene chloride_._._._._._._._._._. 617

Nitromethane _._. 620

Nitrous oxide _..._. 626

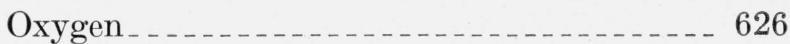

Phosphorus trifluoride__._._._._._._._. 627

Phosphorus sulfatrifluoride_._. _. _..._. _ 628

Propyne _. _. _ _ _ 618

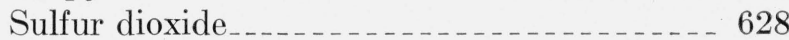

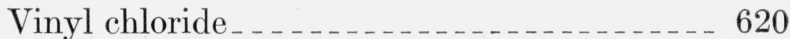

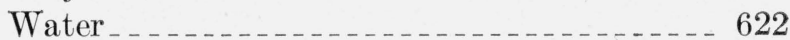

Washington, February 14, 1949. 